JOURNAL OF THE

AMERICAN MATHEMATICAL SOCIETY

Volume 10, Number 3, July 1997, Pages 513-552

S 0894-0347(97)00234-8

\title{
COHOMOLOGY OF UNITS AND $L$-VALUES AT ZERO
}

\author{
JÜRGEN RITTER AND ALFRED WEISS \\ Dedicated to A. Fröhlich on his $80^{\text {th }}$ birthday
}

Let $K / k$ be a finite Galois extension of number fields with Galois group $G$, and let $S$ be a finite $G$-stable set of primes of $K$ containing all archimedean primes. We denote the $G$-module of $S$-units of $K$ by $E=E_{S}$ and let $\Delta S$ be the kernel of the augmentation map $\mathbb{Z} S \rightarrow \mathbb{Z}$ which sends each basis element $\mathfrak{p} \in S$ to 1 . We are concerned with invariants of $K / k$ which are associated to a $G$-homomorphism $\varphi: \Delta S \rightarrow E$ inducing $\mathbb{Q} \otimes \Delta S \simeq \mathbb{Q} \otimes E$

These invariants were defined by Tate [Ta2] and Chinburg [Ch1] when $S$ is large, i.e. when $S$ contains all ramified primes of $K / k$ and the $S$-class group $c l=c l_{S}$ of $K$ is trivial. There are two of them and each is a function of the complex characters $\chi$ of $G$. The first, $A_{\varphi}$, has $A_{\varphi}(\chi)$ equal to the Tate regulator at $\chi$ divided by the leading coefficient $c(\chi)$ of the Taylor expansion at $s=0$ of the Artin $L$-function $L(s, \chi)$ with the Euler factors at primes of $S$ omitted. According to Stark's conjecture [St] in Tate's form, $A_{\varphi}$ takes algebraic values which satisfy

$$
A_{\varphi}\left(\chi^{\sigma}\right)=A_{\varphi}(\chi)^{\sigma}
$$

for all automorphisms $\sigma$ of $\mathbb{C}$.

To describe the second invariant $\mathfrak{q}_{\varphi}$ we fix a subfield $F$ of $\mathbb{C}$ so that $F / \mathbb{Q}$ is finite Galois with Galois group $\Gamma$ and so that every representation of $G$ is realizable over $F$. Let $\mathfrak{o}$ be the ring of integers of $F$. The fields $K / k$ and $F$ are related only in $F$ housing the invariants which we attach to $K / k$. Now $\mathfrak{q}_{\varphi}(\chi)$ is a fractional ideal of $\mathfrak{o}$ which comes from the cohomology of $E$. Its definition requires an exact sequence

$$
E \mapsto A \rightarrow B \rightarrow \Delta S
$$

in which $A, B$ are finitely generated cohomologically trivial $G$-modules and which has been constructed by Tate [Ta1] for large $S$.

The main result of this paper is

Theorem A. Suppose $K / k$ is contained in a cyclotomic extension of $\mathbb{Q}$. If 2 does not ramify in $K$, then

$$
\mathfrak{q}_{\varphi}(\chi)=A_{\varphi}(\check{\chi}) \mathfrak{o}
$$

for all characters $\chi$ of $G$ (where $\check{\chi}$ denotes the contragredient character). Without the ramification hypothesis about 2 the equation remains true on replacing $\mathfrak{o}$ by $\mathfrak{o}\left[\frac{1}{2}\right]$.

Received by the editors March 28, 1996.

1991 Mathematics Subject Classification. Primary 11R42; Secondary 11R33, 11R27.

Key words and phrases. Chinburg-Stark conjecture, Galois structure of units.

We acknowledge financial support provided by the DFG and by NSERC. Moreover, we appreciate having been offered a pleasant stay at the Fields Institute in Waterloo, where the first part of the paper was written. 
This was conjectured by Chinburg [Ch1] to hold for completely general $K / k$ after Tate [Ta2] had proved it for all $\mathbb{Q}$-valued characters $\chi$. The notion of relating $L$-values and orders of certain cohomology groups has also been addressed, in two different formalisms, by Lichtenbaum [Li2] and Fröhlich [Fr2]. For connections to Tate's setting see [Ch2, So] and [RW1] respectively. A consequence of Theorem A is Chinburg's root number conjecture [Ch3, p.358] modulo $D(\mathbb{Z} G)$ for the extensions $K / k$ of the theorem. According to $[\mathrm{GH}]$ however, this can also be obtained by different methods.

The basic idea of our proof is to generalize the definitions of $A_{\varphi}, \mathfrak{q}_{\varphi}$ to arbitrary sets $S$ and so also to the smallest possible $S$ which is the set of all archimedean primes. This eventually allows us to involve the Main Conjecture of Iwasawa theory via Leopoldt's conjecture and Fröhlich's relation between resolvents and Galois Gauss sums. Our difficulties with the prime 2 are due entirely to the present status of the Main Conjecture. Much of the proof is completely general and some of it is capable of generalization. However the first barrier to a generalization is that we need to use Stark's conjecture in a very explicit form and not just the Galois equivariance above.

Our generalization to small sets $S$ starts from [RW2] where a general Tate sequence

$$
E \longmapsto A \rightarrow B \rightarrow \nabla
$$

was constructed. Here $A, B$ are still cohomologically trivial but $\nabla$ is more complicated than $\Delta S:$ there is an exact sequence

$$
c l \longmapsto \nabla \rightarrow \bar{\nabla}
$$

where $\bar{\nabla}$ is a $\mathbb{Z} G$-lattice of known structure. Letting $r=r_{S}$ be the number of $G$-orbits of ramified primes of $K / k$ which are not in $S$, then the invariants are now attached to G-isogenies

$$
\varphi: \nabla \rightarrow \tilde{E}=E \oplus(\mathbb{Z} G)^{r},
$$

which are $G$-homomorphisms with finite kernel and cokernel.

By means of the above sequence we obtain a generalized $\mathfrak{q}_{\varphi}$. We then adapt $A_{\varphi}$ to these $\varphi$ in order to establish

Theorem B. For arbitrary $K / k$ the ratio $\mathfrak{a}(\chi)=\left(A_{\varphi}(\check{\chi})\right) / \mathfrak{q}_{\varphi}(\chi)$ is independent of $\varphi$ and $S$.

This result does not even depend on Stark's conjecture on interpreting $\mathfrak{a}(\chi)$, as in $[\mathrm{Fr} 2]$, as a rank- $1 \mathfrak{o}$-module on $\mathbb{C}$, i.e. a product $\mathfrak{a} \cdot z$ with $\mathfrak{a}$ a fractional $\mathfrak{o}$-ideal and $z \neq 0$ in $\mathbb{C}$. The proof of Theorem B comprises the first seven sections of the paper. The first two sections are preliminary: $\S 1$ makes a crucial local construction and $\S 2$ is a review of the Tate sequence for small $S$. In $\S 3$ we make the definitions of $A_{\varphi}, \mathfrak{q}_{\varphi}$ for small $S$ and then break the proof of Theorem B into three steps in $\S 4$. Each of $\S 5, \S 6, \S 7$ then completes one of these steps. The last step 3 is essentially in [Ch1], the middle step 2 works because the definition of $A_{\varphi}$ is devised to make it so. That step 1 then works is surprising, but essential for what follows.

The next two sections prepare for the proof of Theorem A by bringing Theorem $\mathrm{B}$ to bear. For this proof we need to construct explicit isogenies $\varphi: \nabla \rightarrow \tilde{E}$. In $\S 8$, which works for arbitrary $K / k$, we show how to get a $\varphi$ from an isogeny $\underline{\varphi}: \Delta S \rightarrow E$ when $S$ is not large, and reduce the calculation of $\mathfrak{a}_{\varphi}(\chi)$ to $\underline{\varphi}$ up to fractional $\mathfrak{o}$-ideal factors which divide $|G|$. And then in $\S 9$, assuming now that 
Stark's conjecture holds, we use the induction and inflation properties of $\mathfrak{a}$, which hold for small $S$ because of Theorem B, to reduce the study of prime factors of $\mathfrak{a}(\chi)$ above a rational prime $l$ to the case of $l$-adic linear characters $\chi$ for which $l$ does not divide $|G / \operatorname{ker}(\chi)|$. The problem of Theorem $\mathrm{A}$ is thus translated into establishing an $l$-adic analogue $\mathfrak{a}^{(l)}(\chi)=(1)$ for all primes $l$.

Finally in $\S 10$, we specialize to $K / k$ contained in a cyclotomic field $\mathbb{Q}(\zeta)$, and construct an explicit isogeny $\underline{\varphi}: \Delta S \rightarrow E$ on taking $S$ to be the set of all infinite primes of $K$. This isogeny $\varphi$ comes naturally from the construction of Ramachandra units, which allows us to eliminate the complex $L$-value part $c_{S}(\chi)$ of $\mathfrak{a}^{(l)}$. Then, in $\S 11$, we apply the preliminary results of $\S \S 8,9$ to give the proof of Theorem A. For each prime $l$ we distinguish three cases according as $\chi$ is odd, imaginary or even. In the odd case the assertion $\mathfrak{a}^{(l)}(\chi)=1$ amounts to a conjecture of Brumer which has been proved by Wiles [Wi2]. The imaginary case, for which $k$ is not real, is a sort of analogue for $l=2$ and has been settled by Greither [Gc]. Both cases are applications of the Main Conjecture [MW, Wi1].

Most of the remaining effort is concerned with the even case in which we are left with proving a Ramachandra-Gras conjecture, i.e. the reformulation of a conjecture of Gras for Ramachandra units. Following the strategy of Greenberg [Gr1] we are reduced to computing a certain module index and to making connections again with the Main Conjecture. The module index calculation, in $\$ 12$, depends on the $l$-adic logarithm, which, on the one hand, brings in the $l$-adic $L_{l}(1, \chi)$, and on the other, the $l$-adic additive Galois module structure. Since $l$ is prime to the group order, we are in the tame situation and can apply results of Fröhlich. In $\S 13$ the main results of [Wi1] are brought to bear to finish the proof.

\section{Local CONSTRUCTIONS}

In this section only the extension $K / k$ is not the given one but its completion at a finite prime $\mathfrak{p}$. In particular, $G$ now is the old decomposition group at $\mathfrak{p}$. Denote by $I$ its inertia subgroup and by $\phi$ the Frobenius automorphism of the corresponding residue field extension; set $\bar{G}=G / I, e=|I|, f=|\bar{G}|$. As always, if $H$ is a group, $\Delta H$ is the augmentation ideal in the integral group ring $\mathbb{Z} H$, and $\hat{H}=\sum_{h \in H} h \in \mathbb{Z} H$.

Definition $([\mathrm{GW}])$. The inertial lattice $W$ attached to $K / k$ is the free $\mathbb{Z}$-module on the basis

$$
w_{g}=\left(g-1,1+\phi+\ldots+\phi^{a(g)-1}\right) \in \Delta G \oplus \mathbb{Z} \bar{G}, g \in G,
$$

where $\bar{g}=(g \bmod I)=\phi^{a(g)}$ and $1 \leq a(g) \leq f$. The $G$-action is given by

$$
h w_{g}=w_{h g}-w_{h}+a_{h, g} w_{1}, h \in G,
$$

with $a_{h, g}=0$ or 1 according as $a(h)+a(g) \leq f$ or $>f$.

Observe that $W \simeq \mathbb{Z} G$ if $K / k$ is unramified.

The inertial lattice $W$ comes equipped with a short exact sequence $\mathbb{Z} \longmapsto W \rightarrow$ $\Delta G, 1 \mapsto w_{1}$, from which $\mathbb{Q} \otimes_{\mathbb{Z}} W \simeq \mathbb{Q} G$ follows. The $\mathbb{Z}$-dual of this sequence is

$$
\Delta G^{0} \longmapsto W^{0} \stackrel{\iota}{\rightarrow} \mathbb{Z}
$$

The map $\iota$, which, of course, is the evaluation at $w_{1}$, is called the inertial augmentation. 
Define the elements $\eta, \kappa, \rho \in W^{0}=\operatorname{Hom}(W, \mathbb{Z})$ by $\eta\left(w_{g}\right)=\left\{\begin{array}{l}1, \\ 0,\end{array} \quad \kappa\left(w_{g}\right)=\left\{\begin{array}{ccc}0 & \text { if } & g=1, \\ -1 & \text { if } & g \neq 1,\end{array} \quad \rho\left(w_{g}\right)=\left\{\begin{array}{cll}1 & \text { if } & g=1, \\ 1+e & \text { if } & 1 \neq g \in I, \\ e & \text { if } & g \notin I .\end{array}\right.\right.\right.$

Also, if $V$ is a $\mathbb{C} G$-module, let $\check{V}=\operatorname{Hom}_{\mathbb{C}}(V, \mathbb{C})$ be its contragredient.

Proposition 1. (a) The image of $\Delta G^{0}$ in $W^{0}$ is generated by $\kappa$ as a $\mathbb{Z} G$-module. Also $\hat{G} \eta=\hat{G} \rho$.

(b) If $\beta \in W^{0}$ has augmentation 0 , then $\beta=\left(\sum_{g \neq 1} x_{g} g\right) \kappa$ with $x_{g}=\beta\left(w_{g}\right)$. In particular, there exists $y \in \mathbb{Z} G$, unique up to adding a multiple of $\hat{G}$, such that $y \kappa=(|G|-\hat{G}) \rho$. Also we have $(|G|-\hat{G}) \eta=-e \sum_{g \neq 1} a(g) g \kappa$.

(c) Given a $\mathbb{C} G$-module $V$, let $y_{V}$ denote the right action $f \mapsto f y$ by the above $y$ on $\operatorname{Hom}_{\mathbb{C} G}\left(\check{V}, \mathbb{C} \otimes \Delta G^{0}\right)$, where $(f y)(\check{v})=f(\check{v})$ y for $f \in \operatorname{Hom}_{\mathbb{C} G}\left(\check{V}, \mathbb{C} \otimes \Delta G^{0}\right), \check{v} \in$ V. Then

$$
\operatorname{det}\left(y_{V}\right) \operatorname{det}\left(1-\phi \mid V^{I} / V^{G}\right)=(-e|G|)^{\operatorname{dim} V-\operatorname{dim} V^{G}} .
$$

(d) The $\mathbb{Z} G$-submodule of $W^{0}$ generated by $\rho$ has finite index.

Proof. (a) Denote by $\left\{\eta_{g}: g \in G\right\}$ the dual basis of $W^{0}$ to $\left\{w_{g}: g \in G\right\}$; thus $\eta=\eta_{1}$. Then $\left\{\eta_{g}: 1 \neq g \in G\right\}$ is a $\mathbb{Z}$-basis for the kernel of the inertial augmentation. Since for $g \neq 1,(g \kappa)\left(w_{h}\right)=\kappa\left(g^{-1} w_{h}\right)=\kappa\left(w_{g^{-1} h}-w_{g^{-1}}+a_{g^{-1}, h} w_{1}\right)=\kappa\left(w_{g^{-1} h}\right)+1$, we have $g \kappa=\eta_{g}$. Moreover, $\iota(\kappa)=0$ and, as $\hat{G} w_{h}=e a(h) w_{1},(\hat{G} \nu)\left(w_{g}\right)=\nu\left(\hat{G} w_{g}\right)=$ $e a(g) \iota(\nu)$ for $\nu \in W^{0}$.

Taking $\nu=\kappa$ yields $\left(\sum_{g \in G} a_{g} g\right) \kappa=\sum_{g \neq 1}\left(a_{g}-a_{1}\right) g \kappa=\sum_{g \neq 1}\left(a_{g}-a_{1}\right) \eta_{g}$, and this equals 0 if, and only if, $a_{g}=a_{1}$ for all $g$. Hence $\mathbb{Z} G / \hat{G} \simeq \mathbb{Z} G \kappa$. Also $\nu=\rho-\eta$ has vanishing inertial augmentation.

(b) From (a) we obtain $\beta=\left(\sum_{g \neq 1} x_{g} g\right) \kappa=\sum_{g \neq 1} x_{g} \eta_{g}$. Evaluating at $w_{h}$ shows $x_{h}=\beta\left(w_{h}\right)$. Substituting $(|G|-\hat{G}) \rho$ for $\beta$ yields $x_{h}=e|G|$ or $e|G|-e a(h)$ according as $1 \neq h \in I$ or $h \notin I$, since $(\hat{G} \rho)\left(w_{h}\right)=e a(h)$.

We subtract $|G|(e-1) \hat{G}$ from $\sum_{h \neq 1} x_{h} h$ with the above $x_{h}$ and get the element $y=e \sum_{i=1}^{f-1} i \phi^{-i} \hat{I}-|G|(e-\hat{I})$, where, by abuse of notation, $\phi^{-i}$ is some representative in $G$ for $\phi^{-i} \in \bar{G}$. Here we have observed that $a(g)=f-i$, if $g \equiv \phi^{-i}$ $\bmod I$. Since $\hat{G} \kappa=0, y \kappa=(|G|-\hat{G}) \rho$. The case $\beta=(|G|-\hat{G}) \eta$ is similar.

(c) $\check{V}$ and $\Delta G^{0}$ are left $G$-modules by the usual contragredient convention, so, for example, $(g \check{v})(v)=\check{v}\left(g^{-1} v\right), v \in V, \check{v} \in \check{V}, g \in G$. Since $\Delta G$ is a right $G$-module as well, $\Delta G^{0}$ actually carries a $(G, G)$-bimodule structure: $\left[g d^{0} h\right](d)=$ $d^{0}\left(g^{-1} d h^{-1}\right), d^{0} \in \Delta G^{0}, d \in \Delta G, g, h \in G$. This induces the right $\mathbb{C} G$-structure on $\operatorname{Hom}_{\mathbb{C} G}\left(\tilde{V}, \mathbb{C} \otimes \Delta G^{0}\right)$ used in the assertion.

We first claim that we have isomorphisms

$$
\operatorname{Hom}_{\mathbb{C} G}\left(\check{V}, \mathbb{C} \otimes \Delta G^{0}\right) \stackrel{\cong}{\rightarrow} \operatorname{Hom}_{\mathbb{C} G}(\mathbb{C} \otimes \Delta G, V) \stackrel{\cong}{\rightarrow} V / V^{G} .
$$

The second one is the evaluation of $f^{\prime} \in \operatorname{Hom}_{\mathbb{C} G}(\mathbb{C} \otimes \Delta G, V)$ at the idempotent $1-\frac{\hat{G}}{|G|}$ which projects to 0 in $V^{G}$. To describe the first one, write $\langle\cdot, \cdot\rangle_{V}$ for the canonical pairing $\check{V} \times V \rightarrow \mathbb{C}$ and $\langle\cdot, \cdot\rangle_{\Delta}$ for the one with respect to $\mathbb{C} \otimes \Delta G$. Then the map in question sends $f \in \operatorname{Hom}_{\mathbb{C} G}\left(\check{V}, \mathbb{C} \otimes \Delta G^{0}\right)$ to $f^{\prime} \in \operatorname{Hom}_{\mathbb{C} G}(\mathbb{C} \otimes \Delta G, V)$ where

$$
\left\langle\check{v}, f^{\prime}(d)\right\rangle_{V}=\langle f(\check{v}), d\rangle_{\Delta} \text { for } d \in \mathbb{C} \otimes \Delta G, \check{v} \in \check{V}
$$


It follows that $(f y)^{\prime}(d)=f^{\prime}(d \check{y})$, where $\check{y}$ is obtained from $y$ by the involution on $\mathbb{C} G$ induced by $g \mapsto g^{-1}, g \in G$. This holds because

$$
\left\langle\check{v},(f y)^{\prime}(d)\right\rangle_{V}=\langle(f y)(\check{v}), d\rangle_{\Delta}=\langle f(\check{v}) y, d\rangle_{\Delta}=\langle f(\check{v}), d \check{y}\rangle_{\Delta}=\left\langle\check{v}, f^{\prime}(d \check{y})\right\rangle_{V} .
$$

Thus the right action of $y$ on $\operatorname{Hom}_{\mathbb{C} G}\left(\check{V}, \mathbb{C} \otimes \Delta G^{0}\right)$ corresponds to the left action of $\check{y}$ on $V / V^{G}$. Namely, $f y$ corresponds to $f^{\prime}\left(\left(1-\frac{\hat{G}}{|G|}\right) \check{y}\right)=f^{\prime}\left(\check{y}\left(1-\frac{\hat{G}}{|G|}\right)\right)=\check{y} f^{\prime}\left(1-\frac{\hat{G}}{|G|}\right)$, as $1-\frac{\hat{G}}{|G|} \in \mathbb{C} G$ is central and $f^{\prime}$ is a $G$-homomorphism.

We have arrived at $\operatorname{det}\left(y_{V}\right)=\operatorname{det}\left(\check{y} \mid V / V^{G}\right)$. Because of $y=e \sum_{i=1}^{f-1} i \phi^{-i} \hat{I}-$ $|G|(e-\hat{I})$ the action of $\check{y}$ on $V / V^{G}$ fits into the commutative diagram

$$
\begin{array}{cccc}
V^{I} / V^{G} & \longmapsto & V / V^{G} & \rightarrow V / V^{I} \\
\downarrow \tilde{y} & & \downarrow \check{y} & \downarrow-|G| e \\
V^{I} / V^{G} & & \longmapsto / V^{G} & \rightarrow V / V^{I}
\end{array}
$$

with multiplication by $-|G| e$ on the right vertical arrow and multiplication by $\tilde{y}=$ $e^{2} \sum_{i=1}^{f-1} i \phi^{i}$ on the left one. Hence $\operatorname{det}\left(\check{y} \mid V / V^{G}\right)=(-e|G|)^{\operatorname{dim} V / V^{I}} \cdot \operatorname{det}\left(\tilde{y} \mid V^{I} / V^{G}\right)$ and $\tilde{y}(1-\phi)=e^{2} \sum_{i=1}^{f-1} i \phi^{i}(1-\phi)=e^{2}(\langle\hat{\phi}\rangle-f)$, so

$$
\begin{aligned}
& \operatorname{det}\left(\check{y} \mid V / V^{G}\right) \operatorname{det}\left(1-\phi \mid V^{I} / V^{G}\right)=(-e|G|)^{\operatorname{dim} V / V^{I}}(-e|G|)^{\operatorname{dim} V^{I} / V^{G}} \\
& \quad=(-e|G|)^{\operatorname{dim} V / V^{G}},
\end{aligned}
$$

since $\langle\hat{\phi}\rangle V^{I} \subset V^{G}$.

(d) By (c), $y \in \mathbb{Q} G / \hat{G} \mathbb{Q} G$ is a unit. Thus $y \kappa=(|G|-\hat{G}) \rho$ generates a submodule of finite index in $\Delta G^{0}=\mathbb{Z} G \kappa$. Since $\rho$ itself has inertial augmentation $\neq 0$, the assertion follows readily.

Remark. Eventually, in $\S 6$, the point of $\S 1$ will be that $\operatorname{det}\left(1-\phi_{\mathfrak{p}} \mid V^{I_{\mathfrak{p}}} / V^{G_{\mathfrak{p}}}\right)$ is an Euler factor at $s=0$.

\section{The Tate Sequence; $\nabla$ modulo torsion}

We return to our original notation in which $K / k$ is an extension of number fields and, from now on, label the objects defined in $\S 1$ by the appropriate primes. In particular, $G_{\mathfrak{p}}, W_{\mathfrak{p}}, W_{\mathfrak{p}}^{0} \stackrel{\iota_{\mathfrak{p}}}{\longrightarrow} \mathbb{Z}$ are the decomposition group, the inertial lattice and the inertial augmentation at $\mathfrak{p}$, respectively. Once and for all we fix a choice * of $G$-orbit representatives in the set of all primes of $K$. For a $G$-stable subset $S$ then $S_{*}$ is the intersection of $S$ and $*$.

Set

$$
\begin{aligned}
& S^{\text {ram }}=\{\mathfrak{p} \text { ramified in } K / k, \mathfrak{p} \notin S\}, \\
& S_{\text {ram }}=S \cup S^{\text {ram }}, \\
& { }^{0} W={ }^{0} W_{S}=\bigoplus_{\mathfrak{p} \in S_{*}^{\text {ram }} \operatorname{ind}_{G_{\mathfrak{p}}}^{G} W_{\mathfrak{p}}^{0},} \\
& { }^{0} \Delta={ }^{0} \Delta_{S}=\bigoplus_{\mathfrak{p} \in S_{*}^{\text {ram }} \operatorname{ind}_{G_{\mathfrak{p}}}^{G} G_{\mathfrak{p}}^{0},} \\
& \bar{\nabla}=\bar{\nabla}_{S}=\operatorname{ker}\left(\mathbb{Z} S \oplus{ }^{0} W \stackrel{\iota^{\prime}}{\longrightarrow} \mathbb{Z}\right),
\end{aligned}
$$

where $\iota^{\prime}$ is the augmentation on $\mathbb{Z} S$ and $\operatorname{ind}_{G_{\mathfrak{p}}}^{G} \iota_{\mathfrak{p}}$ (followed by the augmentation $\left.\operatorname{ind}_{G_{\mathfrak{p}}}^{G} \mathbb{Z} \rightarrow \mathbb{Z}\right)$ on $\operatorname{ind}_{G_{\mathfrak{p}}}^{G} W_{\mathfrak{p}}^{0}$. 
The right hand end $\nabla$ in the Tate sequence associated to $S$,

$$
E_{S} \longmapsto A \rightarrow B \rightarrow \nabla
$$

originates from a unique extension

$$
c l_{S} \longmapsto \nabla \rightarrow \bar{\nabla}
$$

which is described in [RW2]; however, for the purposes of this paper it usually suffices to know $\nabla$ modulo its torsion $c l_{S}$, i.e. $\bar{\nabla}$. The $\mathbb{Z} G$-modules $A, B$ are cohomologically trivial and stably free, respectively.

In the following sections we need to compare Tate sequences for sets $S_{1} \subset S_{2}$. In order to do so we use

Theorem 2. Given $S_{1} \subset S_{2}$, it is possible to choose Tate sequences for $S_{1}$ and $S_{2}$ as the rows in the commutative diagram

$$
\begin{aligned}
& E_{1} \longmapsto A_{1} \rightarrow B_{1} \rightarrow \nabla_{1} \\
& \downarrow \downarrow \downarrow \downarrow \downarrow \downarrow \text { in which the outer vertical } \\
& E_{2} \longmapsto A_{2} \rightarrow B_{2} \rightarrow \nabla_{2} \quad \text { maps are the natural ones. }
\end{aligned}
$$

Proof. The theorem is actually a direct consequence of the construction of the Tate sequence. In its proof all references are to [RW2]. Choose a sufficiently large set $S^{\prime}$ of primes containing $S_{2}$ and look at the diagram

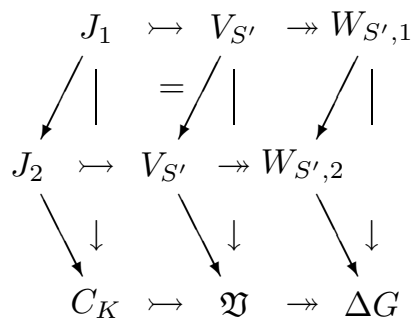

in which $J_{i}$ denotes the $S_{i}$-ideles and $C_{K}$ the idele class group of $K$, and where

$$
W_{S^{\prime}, i}=\left(\bigoplus_{\mathfrak{p} \in S_{*, i}} \operatorname{ind}_{G_{\mathfrak{p}}}^{G} \Delta G_{\mathfrak{p}}\right) \oplus\left(\bigoplus_{\mathfrak{p} \in S_{*}^{\prime} \backslash S_{*, i}} \operatorname{ind}_{G_{\mathfrak{p}}}^{G} W_{\mathfrak{p}}\right) .
$$

For $V_{S^{\prime}}$ and $\mathfrak{V}$ see $\S 1$ of [RW2] (as usual); they, together with the rows in the above diagram, are determined by the local and global fundamental classes. The left and right triangles are the obvious ones. The top face is built from the local diagrams of Proposition 2. Theorem 1 provides a surjective map $V_{S^{\prime}} \rightarrow \mathfrak{V}$ making the diagram commute. The modules $V_{S^{\prime}}, \mathfrak{V}$ are cohomologically trivial and so the snake lemma yields

$$
\begin{array}{ccccccc}
E_{1} & \longmapsto & A_{1} & \rightarrow & R_{S^{\prime}, 1} & \rightarrow & c l_{1} \\
\downarrow & & \downarrow & & \downarrow & & \downarrow \\
E_{2} & \longmapsto & A_{2} & \rightarrow & R_{S^{\prime}, 2} & \rightarrow & c l_{2}
\end{array}
$$

with cohomologically trivial $A_{1}, A_{2}$. 
We next build the commutative prism

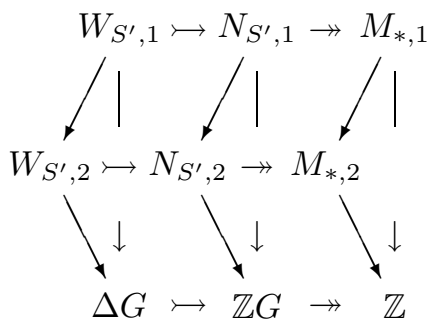

in which the front and the back face are as in diagram 4 (of [RW2]) and the top face is assembled from local diagrams mostly with identity maps; the only complicated case being $\mathfrak{p} \in\left(S_{2} \cap S_{1}^{\mathrm{ram}}\right)_{*}$ when the relevant local diagram is described in Lemma 5c). It follows that $M_{*, 1} \rightarrow M_{*, 2}$ is induced by the inertial augmentations. On taking kernels we obtain the top face of



its left face is taken from the second diagram and the rest follows by pushing out. Applying the construction at the end of $\S 4$ then yields the claim of the theorem.

We close this section by presenting two useful exact sequences involving $\bar{\nabla}$ :

$$
\Delta S \longmapsto \bar{\nabla} \rightarrow{ }^{0} W,{ }^{0} \Delta \longmapsto \bar{\nabla} \rightarrow \Delta S_{\text {ram }} .
$$

Here, $\bar{\nabla} \rightarrow{ }^{0} W$ is the restriction of the projection $\mathbb{Z} S \oplus{ }^{0} W \rightarrow{ }^{0} W$ and the map $\bar{\nabla} \rightarrow \Delta S_{\text {ram }}$ is, on the one hand, induced by the embedding $S \subset S_{\text {ram }}$ and, on the other hand, by $\operatorname{ind}_{G_{\mathfrak{p}}}^{G} W_{\mathfrak{p}}^{0} \rightarrow \operatorname{ind}_{G_{\mathfrak{p}}}^{G} \mathbb{Z}$.

As a consequence,

$$
\mathbb{Q} \otimes_{\mathbb{Z}} \nabla \simeq \mathbb{Q} \otimes_{\mathbb{Z}} \bar{\nabla} \simeq \mathbb{Q} \otimes_{\mathbb{Z}} \Delta S \oplus \mathbb{Q} \otimes_{\mathbb{Z}}{ }^{0} W \simeq \mathbb{Q} \otimes_{\mathbb{Z}}\left(E \oplus \mathbb{Z} G^{r}\right)
$$

with the $r$ that is already defined in the introduction, i.e.

$r=r_{S}=$ number of $G$-orbits of ramified primes of $K / k$ which are not in $S$.

Remark. For a discussion of the dependence on the choice $*$ see [RW2, Theorem 6].

\section{Definition of $q$-Index and $A$ for small $S$}

Let $S$ be arbitrary, put $r=r_{S}$ and choose an isogeny $\varphi: \nabla \rightarrow \tilde{E}=E \oplus \mathbb{Z} G^{r}$. The definitions we are going to make below generalize those of Tate [Ta2] for large $S$.

We define the $q$-index $\mathfrak{q}_{\varphi}$. 
Let $\chi$ be a character of $G$ and $V=V_{\chi}$ be an $F G$-module affording $\chi$ and $M$ an o $G$-lattice on $V$. Following Tate [Ta2, p.60] when $S$ is large, we abbreviate by $\varphi_{M}$ the composite map

$$
\varphi_{M}: \operatorname{Hom}_{\mathfrak{o}}(M, \nabla)_{G} \stackrel{\hat{G}}{\longrightarrow} \operatorname{Hom}_{\mathfrak{o}}(M, \nabla)^{G} \stackrel{\varphi}{\longrightarrow} \operatorname{Hom}_{\mathfrak{o}}(M, \tilde{E})^{G}
$$

from $G$-coinvariants to $G$-invariants. We resolve the obvious difficulty with the above notation by adopting the convention that $\nabla, \tilde{E}$ are to be $\operatorname{read} \mathfrak{o} \otimes_{\mathbb{Z}} \nabla, \mathfrak{o} \otimes_{\mathbb{Z}} \tilde{E}$. Indeed we will use this convention of letting the context determine the obvious extension of scalars rather consistently throughout the rest of the paper.

Definition. $\mathfrak{q}_{\varphi}(\chi)=\ell_{\mathfrak{o}}\left(\operatorname{coker}\left(\varphi_{M}\right)\right) / \ell_{\mathfrak{o}}\left(\operatorname{ker}\left(\varphi_{M}\right)\right)$, where the length $\ell_{\mathfrak{o}}(X)$ of a finite $\mathfrak{o}$-module $X$ is $\ell_{\mathfrak{o}}(X)=\prod_{i=0}^{m} \mathfrak{l}_{i}$, if $X=X_{0} \supset X_{1} \supset \ldots \supset X_{m} \supset X_{m+1}=0$ is a composition series with $X_{i} / X_{i+1} \simeq \mathfrak{o} / \mathfrak{l}_{i}$.

Lemma 3. (a) $\mathfrak{q}_{\varphi}(\chi)$ does not depend on the choices of $F, V$ and $M$.

(b) If $\chi$ is irreducible, $\mathfrak{q}_{\varphi}(\chi)$ is a fractional $\mathfrak{o}$-ideal in $F$ which is generated by a fractional ideal of the field $\mathbb{Q}(\chi)$ of character values of $\chi$.

(c) $\mathfrak{q}_{\varphi}(\chi)^{\gamma}=\mathfrak{q}_{\varphi}\left(\chi^{\gamma}\right)$ for $\gamma \in \Gamma$.

Proof. (a) is shown exactly along the lines of Tate's proof [Ta2, Chapter 2] by just replacing his 4 -term sequence [Ta2, p.54] by

$$
\tilde{E} \mapsto \tilde{A} \rightarrow B \rightarrow \nabla,
$$

which is obtained by adding $\mathbb{Z} G^{r}$, with identity map, to $E$ and $A$.

For (b) and (c) see, for example, [We, Proposition 7].

We next define the generalized $A$-number attached to characters of $G$.

Definition. $A_{\varphi}(\chi)=R_{\varphi}(\chi) /\left(c_{S}(\chi)\left[\prod_{\mathfrak{p} \in S_{*}^{\text {ram }}} e_{\mathfrak{p}} \log (N \mathfrak{p})\right]^{\chi(1)}\right)$.

Here, $c_{S}(\chi)$ is the leading coefficient in the Taylor expansion at zero of the Artin $L$-function for $\chi$ with the Euler factors at $\mathfrak{p} \in S$ removed and $N \mathfrak{p}$ is the absolute norm of $\mathfrak{p}$. So it remains to define the generalized Stark-Tate regulator $R_{\varphi}(\chi)$.

For this we need a generalized Dirichlet map

$$
\tilde{\lambda}: \mathbb{R} \otimes_{\mathbb{Z}} \tilde{E} \stackrel{\simeq}{\longrightarrow} \mathbb{R} \otimes_{\mathbb{Z}} \nabla
$$

which replaces the original Dirichlet isomorphism

$$
\lambda: \mathbb{R} \otimes_{\mathbb{Z}} E \stackrel{\simeq}{\longrightarrow} \mathbb{R} \otimes_{\mathbb{Z}} \Delta S
$$

that takes a unit $u \in E$ to $\sum_{\mathfrak{p} \in S} \log |u|_{\mathfrak{p}} \mathfrak{p}$ with $|u|_{\mathfrak{p}}=$ absolute value of $u$ at $\mathfrak{p}$ normalized in the usual way.

We define $\tilde{\lambda}$ as a dotted map making

$$
\begin{array}{ccccc}
\mathbb{R} \otimes E & \longmapsto & \mathbb{R} \otimes \tilde{E} & \rightarrow & \mathbb{R} G^{r} \\
\downarrow \lambda & & \downarrow \cdot & & \downarrow \tilde{\rho} \\
\mathbb{R} \otimes \Delta S & \longmapsto & \mathbb{R} \otimes \bar{\nabla} & \rightarrow & \mathbb{R} \otimes{ }^{0} W
\end{array}
$$

commute, where $\tilde{\rho}\left(1_{\mathfrak{p}}\right)=-\log (N \mathfrak{p}) \otimes \rho_{\mathfrak{p}}$ on viewing $\mathbb{Z} G^{r}$ as $\underset{\mathfrak{p} \in S_{*}^{\text {ram }}}{\bigoplus} \operatorname{ind}_{G_{\mathfrak{p}}}^{G} \mathbb{Z}_{\mathfrak{p}} ; \rho_{\mathfrak{p}}$ is as in Proposition 1. 
We are now ready to define the regulator $R_{\varphi}(\chi)$ that is attached to a complex character $\chi$ of $G$ and an isomorphism

$$
\varphi: \mathbb{C} \otimes \nabla \longrightarrow \mathbb{C} \otimes \tilde{E} .
$$

To that end let $\check{V}$ be a $\mathbb{C} G$-module affording $\check{\chi}$, the contragredient of $\chi$, and denote by $\left[\tilde{\lambda} \varphi \mid \operatorname{Hom}_{\mathbb{C} G}(\check{V}, \nabla)\right]$ the endomorphism of $\operatorname{Hom}_{\mathbb{C} G}\left(\check{V}, \mathbb{C} \otimes_{\mathbb{Z}} \nabla\right)$ which sends a $\operatorname{map} s$ to $\tilde{\lambda} \varphi s$.

Definition. $R_{\varphi}(\chi)=\operatorname{det}\left[\tilde{\lambda} \varphi \mid \operatorname{Hom}_{\mathbb{C} G}(\check{V}, \nabla)\right]$.

This definition is justified by the following lemma.

Lemma 4. $R_{\varphi}(\chi)$ does not depend on the choice of $\tilde{\lambda}$ making the above diagram commute.

Proof. Namely, two such $\tilde{\lambda}$ (multiplicatively) differ by a map $\nu$ making

$$
\begin{array}{ccccc}
\mathbb{R} \otimes \Delta S & \longmapsto & \mathbb{R} \otimes \bar{\nabla} & \rightarrow & \mathbb{R} \otimes{ }^{0} W \\
\| & & \downarrow \nu & & \| \\
\mathbb{R} \otimes \Delta S & \longmapsto & \mathbb{R} \otimes \bar{\nabla} & \rightarrow & \mathbb{R} \otimes{ }^{0} W
\end{array}
$$

commute. Applying $\operatorname{Hom}_{\mathbb{C} G}\left(\check{V}, \mathbb{C} \otimes_{\mathbb{R}} \cdot\right)$ to the diagram we see that the determinant of the map on $\operatorname{Hom}_{\mathbb{C} G}(\check{V}, \mathbb{C} \otimes \bar{\nabla})$ induced by $\nu$ is 1 .

In the lemma to follow we display an explicit $\tilde{\lambda}$ in terms of an auxiliary map $a$ which often is quite easy to come by. Namely let $a$ be a $\mathbb{Q} G$-map making the following triangle commute:

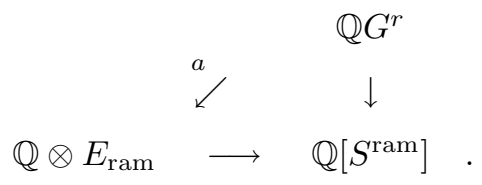

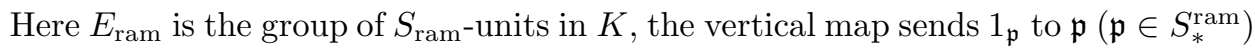
and the horizontal $u \in E_{\text {ram }}$ to $\sum_{\mathfrak{p} \in S_{\text {ram }}} v_{\mathfrak{p}}(u) \mathfrak{p}$ with $v_{\mathfrak{p}}$ denoting the $\mathfrak{p}$-valuation.

Lemma 5. The map $\tilde{\lambda}: \mathbb{R} \otimes \tilde{E} \rightarrow \mathbb{R}[S] \oplus\left(\mathbb{R} \otimes{ }^{0} W\right)$ satisfying $\tilde{\lambda}(u)=\lambda(u)$ for $u \in E$ and $\tilde{\lambda}\left(1_{\mathfrak{p}}\right)=\sum_{\mathfrak{p}^{\prime} \in S} \log \left|a\left(1_{\mathfrak{p}}\right)\right|_{\mathfrak{p}^{\prime} \mathfrak{p}^{\prime}}-\log (N \mathfrak{p}) \otimes \rho_{\mathfrak{p}}$ for $\mathfrak{p} \in S^{\text {ram }}$ takes its values in $\mathbb{R} \otimes \bar{\nabla}$ and induces $\tilde{\rho}$ on $\mathbb{R} G^{r}$.

Proof. The second claim directly follows from $\bar{\nabla} \rightarrow{ }^{0} W$ being the restriction of the projection $\mathbb{Z} S \oplus^{0} W \rightarrow{ }^{0} W$ to $\bar{\nabla}$. In order to see the first one, we compute (inertial) augmentations.

(i) $\sum_{\mathfrak{p}^{\prime} \in S} \log \left|a\left(1_{\mathfrak{p}}\right)\right|_{\mathfrak{p}^{\prime}}+\sum_{\mathfrak{p}^{\prime \prime} \in S^{\text {ram }}} \log \left|a\left(1_{\mathfrak{p}}\right)\right|_{\mathfrak{p}^{\prime \prime}}=0$, as $a\left(1_{\mathfrak{p}}\right) \in \mathbb{Q} \otimes E_{\text {ram }}$ (here we have extended $|\cdot|_{\mathfrak{p}^{\prime \prime}}$ to $\mathbb{Q} \otimes E_{\text {ram }}$ in the obvious way).

(ii) $a\left(1_{\mathfrak{p}}\right)$ has image $\mathfrak{p} \in \mathbb{Q}\left[S^{\text {ram }}\right]$, hence $\left|a\left(1_{\mathfrak{p}}\right)\right|_{\mathfrak{p}^{\prime \prime}}=1$ or $(N \mathfrak{p})^{-1}$ according to $\mathfrak{p}^{\prime \prime} \neq \mathfrak{p}$ or $\mathfrak{p}^{\prime \prime}=\mathfrak{p}$ in $S^{\text {ram }}$. Consequently, $\sum_{\mathfrak{p}^{\prime \prime} \in S^{\text {ram }}} \log \left|a\left(1_{\mathfrak{p}}\right)\right|_{\mathfrak{p}^{\prime \prime}}=-\log (N \mathfrak{p})$. The assertion follows by combining with $\iota_{\mathfrak{p}}\left(\rho_{\mathfrak{p}}\right)=1$.

Remark. Observe that if $S$ is small, then the choice $*$ of $G$-orbit representatives of the primes of $K$ is involved in the map $\varphi$, since $\nabla$ depends on $*$ [RW2], and so there is no ambiguity in writing $R_{\varphi}(\chi)$ or $A_{\varphi}(\chi)$. 


\section{Proof strategy for Theorem B}

Given an isogeny $\varphi: \nabla \rightarrow \tilde{E}$, we define

$$
\mathfrak{a}_{\varphi}(\chi)=A_{\varphi}(\check{\chi}) / \mathfrak{q}_{\varphi}(\chi) .
$$

Proposition 6. If $\varphi_{1}, \varphi_{2}: \nabla \rightarrow \tilde{E}$ are two isogenies, then $\mathfrak{a}_{\varphi_{1}}(\chi)=\mathfrak{a}_{\varphi_{2}}(\chi)$.

Proof. This can be read off from Chinburg's work [Ch1] and, in any case, from the proof of Proposition 8 in [We], but is included for completeness. Here is what we precisely show:

$$
A_{\varphi_{1}}(\check{\chi}) / A_{\varphi_{2}}(\check{\chi}) \text { belongs to } F^{\times} \text {and generates } \mathfrak{q}_{\varphi_{1}}(\chi) / \mathfrak{q}_{\varphi_{2}}(\chi) \text {. }
$$

Since, over $\mathbb{Q}, \varphi_{1}$ and $\varphi_{2}$ become isomorphisms, there exists an automorphism $\theta$ of $\mathbb{Q} \otimes_{\mathbb{Z}} \nabla$ such that $\varphi_{1}=\varphi_{2} \theta$. Choose an $F G$-module $V$ affording the character $\chi$. Then there is a commutative triangle

$$
\begin{array}{ccc}
\operatorname{Hom}_{F G}(V, F \otimes \nabla) & \searrow^{\varphi_{2}} & \\
\uparrow \theta & \varphi_{1} & \operatorname{Hom}_{F G}(V, F \otimes \tilde{E}) \\
\operatorname{Hom}_{F G}(V, F \otimes \nabla) & \nearrow &
\end{array}
$$

with obvious maps. After tensoring the triangle with $\mathbb{C}$ over $F$ and composing with the map $\operatorname{Hom}_{\mathbb{C} G}\left(\mathbb{C} \otimes_{F} V, \mathbb{C} \otimes_{\mathbb{Z}} \tilde{E}\right) \stackrel{\tilde{\lambda}}{\rightarrow} \operatorname{Hom}_{\mathbb{C} G}\left(\mathbb{C} \otimes_{F} V, \mathbb{C} \otimes_{\mathbb{Z}} \nabla\right)$ at the right hand side, we deduce that

$$
R_{\varphi_{1}}(\check{\chi})=\operatorname{det}\left(\theta \mid \operatorname{Hom}_{\mathbb{C} G}\left(\mathbb{C} \otimes_{F} V, \mathbb{C} \otimes_{\mathbb{Z}} \nabla\right)\right) \cdot R_{\varphi_{2}}(\check{\chi}) .
$$

The determinant factor thus belongs to $F^{\times}$and is the quotient $A_{\varphi_{1}}(\check{\chi}) / A_{\varphi_{2}}(\check{\chi})$.

Regarding the quotient of the $q$-indices, we use the commutative diagrams, ${ }^{1}$ for $i=1,2$,

$$
\begin{array}{cccccc}
T_{\nabla} & \longmapsto & \operatorname{Hom}_{\mathfrak{o}}(M, \nabla)_{G} & \rightarrow & L_{\nabla} \\
\frac{\varphi_{i} \downarrow}{T^{E}} & & \varphi_{i, M} \downarrow & & \varphi_{i} \downarrow \\
T^{2} & \longmapsto & \operatorname{Hom}_{\mathfrak{o}}(M, \tilde{E})^{G} & \rightarrow & L^{E}
\end{array}
$$

in which $T_{\nabla}$ and $T^{E}$ are the respective torsion submodules of $\operatorname{Hom}_{\mathfrak{o}}(M, \nabla)_{G}$ and $\operatorname{Hom}_{\mathfrak{o}}(M, \tilde{E})^{G}$, and where $\underline{\varphi}_{i}, \bar{\varphi}_{i}$ are the maps induced by $\varphi_{i, M}$. Here $\operatorname{ker}\left(\bar{\varphi}_{i}\right)=0$, since $L_{\nabla}$ is a lattice and all maps are isogenies.

So by [Ta2, pp.58,59],

$$
\mathfrak{q}_{\varphi_{i}}(\chi)=\frac{\ell\left(\operatorname{coker} \underline{\varphi}_{i}\right)}{\ell\left(\operatorname{ker} \underline{\varphi}_{i}\right)} \cdot \ell\left(\operatorname{coker} \bar{\varphi}_{i}\right), \ell=\ell_{\mathfrak{o}} .
$$

Since $T_{\nabla}$ and $T^{E}$ are o-torsion modules, the exact sequence $\operatorname{ker}\left(\underline{\varphi}_{i}\right) \longmapsto T_{\nabla} \stackrel{\underline{\varphi}_{i}}{\longrightarrow}$ $T^{E} \rightarrow \operatorname{coker}\left(\underline{\varphi}_{i}\right)$ implies that $\ell\left(\operatorname{coker}\left(\underline{\varphi}_{i}\right)\right) / \ell\left(\operatorname{ker}\left(\underline{\varphi}_{i}\right)\right)=\ell\left(T^{E}\right) / \ell\left(T_{\nabla}\right)$. Hence

$$
\mathfrak{q}_{\varphi_{1}}(\chi) / \mathfrak{q}_{\varphi_{2}}(\chi)=\frac{\ell\left(\operatorname{coker}\left(\bar{\varphi}_{1}\right)\right)}{\ell\left(\operatorname{coker}\left(\bar{\varphi}_{2}\right)\right)} .
$$

\footnotetext{
${ }^{1}$ With $\nabla, \tilde{E}$ inside $\mathrm{Hom}_{\mathfrak{o}}$ meaning $\mathfrak{o} \otimes_{\mathbb{Z}} \nabla, \mathfrak{o} \otimes_{\mathbb{Z}} \tilde{E}$ by our convention.
} 
We next exploit the commutative diagrams

$\begin{array}{cccc}\operatorname{Hom}_{\mathfrak{o}}(M, \nabla)_{G} & \stackrel{\varphi_{i, M}}{\longrightarrow} & \operatorname{Hom}_{\mathfrak{o}}(M, \tilde{E})^{G} & \text { where the right ver- } \\ \downarrow \hat{G} & & \downarrow & \text { tical map is just the } \\ \operatorname{Hom}_{F G}(V, F \otimes \nabla) & \stackrel{\varphi_{i}}{\rightarrow} & \operatorname{Hom}_{F G}(V, F \otimes \tilde{E}) & \text { extension of scalars, }\end{array}$

in order to identify $\bar{\varphi}_{i}$ as the restriction of $\varphi_{i}$ to $L_{\nabla}$. In particular, by the triangle above, $\bar{\varphi}_{1}=\bar{\varphi}_{2} \theta$ and so

$$
\begin{aligned}
\mathfrak{q}_{\varphi_{1}}(\chi) / \mathfrak{q}_{\varphi_{2}}(\chi) & =\frac{\left[L^{E}: \bar{\varphi}_{1}\left(L_{\nabla}\right)\right]}{\left[L^{E}: \bar{\varphi}_{2}\left(L_{\nabla}\right)\right]}=\left[\bar{\varphi}_{2}\left(L_{\nabla}\right): \bar{\varphi}_{2} \theta\left(L_{\nabla}\right)\right] \\
& =\left[L_{\nabla}: \theta\left(L_{\nabla}\right)\right]=\operatorname{det} \theta \cdot \mathfrak{o} .
\end{aligned}
$$

This finishes the proof of Proposition 6.

Because of the proposition, $\mathfrak{a}_{\varphi}$ depends only on $S$ rather than on the particular $\varphi$, so we put $\mathfrak{a}_{S}(\chi)=\mathfrak{a}_{\varphi}(\chi)$. Theorem B asserts that $\mathfrak{a}_{S}(\chi)$ is even independent of $S$. Its proof is based on the equality $\mathfrak{a}_{S}(\chi)=\mathfrak{a}_{S^{\prime}}(\chi)$ for any sufficiently large set $S^{\prime}$ containing $S$. So, if $S_{1}$ and $S_{2}$ are two sets of primes, then both are contained in the same sufficiently large $S^{\prime}$ and we get $\mathfrak{a}_{S_{1}}(\chi)=\mathfrak{a}_{S^{\prime}}(\chi)=\mathfrak{a}_{S_{2}}(\chi)$.

The equality $\mathfrak{a}_{S}(\chi)=\mathfrak{a}_{S^{\prime}}(\chi)$ is proved in three steps, each of which will fill an extra section. Step 1 in $\S 5$ concerns adjoining $G$-orbits of primes of absolute degree 1 to $S$. It is always possible to adjoin a set of generators of the $S$-class group of $K$ in this way because it is the primes of degree 1 that carry all of the Dirichlet density in the usual proofs of the existence of primes representing given elements of the class group. So in step 2 , in $\S 6$, we begin with a set $S$ for which $c l_{S}=0$ and enlarge it by adjoining all ramified primes of $K / k$. Finally, in step 3 in $\S 7$, the initial set $S$ is large in the sense of Tate and we must now enlarge it arbitrarily. The arguments used in this last step are not really new, see [Ch1] or [We].

The computations to be carried out in the following three sections will be easier to follow if we make use of some further notation. The Tate cohomology groups $H^{i}\left(G, \operatorname{Hom}_{\mathfrak{o}}(M, X)\right)$, where $i \in \mathbb{Z}, X$ is a finitely generated $\mathbb{Z} G$-module and $M$ a fixed $\mathfrak{o} G$-lattice with character $\chi$, will be denoted by $H^{i}(X)$. Moreover, with respect to evaluating the length of artinian $\mathfrak{o}$-modules we shall mainly work in the Grothendieck group $K_{0} T(\mathfrak{o})$ of the category of finitely generated torsion $\mathfrak{o}$-modules. We write $[T]$ for the element in $K_{0} T(\mathfrak{o})$ induced by the $\mathfrak{o}$-torsion module $T$. Observe that applying $\ell_{\mathfrak{o}}$ to artinian $\mathfrak{o}$-modules yields a homomorphism from $K_{0} T(\mathfrak{o})$ into the fractional o-ideals of $F$. For an isogeny $f: Y \rightarrow Y^{\prime}$ between finitely generated $\mathfrak{o}$-modules we set $[f]=[\operatorname{coker}(f)]-[\operatorname{ker}(f)]$. An example of such an $f$ is the map $t_{M}: \operatorname{Hom}_{\mathfrak{o}}(M, Y)_{G} \stackrel{\hat{G}}{\rightarrow} \operatorname{Hom}_{\mathfrak{o}}(M, Y)^{G} \stackrel{t}{\rightarrow} \operatorname{Hom}_{\mathfrak{o}}\left(M, Y^{\prime}\right)^{G}$ associated to any $G$-isogeny $t: Y \rightarrow Y^{\prime}$. In particular, $\mathfrak{q}_{\varphi}(\chi)=\ell_{\mathfrak{o}}\left(\left[\varphi_{M}\right]\right)$.

We close this section by recalling a formula which follows from the definition of the Euler factors (see [We, Proposition 6]).

\section{Lemma 7.}

$$
c_{S_{1}}(\check{\chi}) / c_{S}(\check{\chi})=\prod_{\mathfrak{p} \in\left(S_{1} \backslash S\right)_{*}}\left(\frac{\log (N \mathfrak{p})}{f_{\mathfrak{p}}}\right)^{\operatorname{dim} \check{V}^{G_{\mathfrak{p}}}} \operatorname{det}\left(1-\phi_{\mathfrak{p}} \mid \check{V}^{I_{\mathfrak{p}}} / \check{V}^{G_{\mathfrak{p}}}\right) \text { for } S_{1} \supseteq S .
$$




\section{STEP 1}

Let $S_{1} \supset S$ be a finite $G$-stable set of primes of $K$ such that the $S_{1}$-class group of $K$ is trivial and such that each $\mathfrak{p} \in S_{1} \backslash S$ has absolute degree 1 . We use the subscript 1 in order to distinguish the objects associated to $S_{1}$ from those to $S$. We have $S_{1}^{\mathrm{ram}}=S^{\mathrm{ram}}$, and the notation ${ }^{0} W$ is unambiguous as is the number $r$. Observe that $\mathbb{Z}\left[S_{1} \backslash S\right]$ is $\mathbb{Z} G$-free. The inclusion $\mathbb{Z} S \subset \mathbb{Z} S_{1}$ together with the identity map on ${ }^{0} \mathrm{~W}$ induces the short exact sequence

$$
\bar{\nabla} \longmapsto \nabla_{1} \rightarrow \mathbb{Z}\left[S_{1} \backslash S\right] .
$$

The map $u \mapsto \sum_{\mathfrak{p} \in S_{1} \backslash S} v_{\mathfrak{p}}(u) \mathfrak{p}$ injects $E_{1} / E$ into $\mathbb{Z}\left[S_{1} \backslash S\right]$ and has cokernel $\mathrm{cl}$, the $S$-class group of $K$. Namely, if $\prod_{\mathfrak{p} \in S_{1} \backslash S} \mathfrak{p}^{b_{\mathfrak{p}}}=(1)$ in $c l$, then there exists a $b \in K$ with $v_{\mathfrak{p}}(b)=b_{\mathfrak{p}}$ for $\mathfrak{p} \notin S$ and so, in particular, $v_{\mathfrak{p}}(b)=0$ for $\mathfrak{p} \notin S_{1}$, i.e. $b \in E_{1}$. Since the $S_{1}$-class group of $K$ is trivial, the cokernel is all of $c l$. Hence the multiplication by $h=|c l|$ on $\mathbb{Z}\left[S_{1} \backslash S\right]$ has its image in $E_{1} / E$. The induced map $\mathbb{Z}\left[S_{1} \backslash S\right] \rightarrow E_{1} / E$ is denoted by $h^{\prime}$ :

$$
\begin{aligned}
& \mathbb{Z}\left[S_{1} \backslash S\right]=\mathbb{Z}\left[S_{1} \backslash S\right] \\
& \downarrow h^{\prime} \quad \downarrow h \\
& E_{1} / E \quad \longmapsto \mathbb{Z}\left[S_{1} \backslash S\right] \quad \rightarrow \quad c l .
\end{aligned}
$$

Start with an isogeny $\varphi: \nabla \rightarrow \tilde{E}$ which vanishes on the torsion of $\nabla$ and so induces $\bar{\varphi}: \bar{\nabla} \rightarrow \tilde{E}$ :

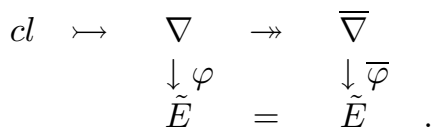

The existence of a map $\varphi_{1}$ in the commutative diagram below is due to $\mathbb{Z}\left[S_{1} \backslash S\right]$ being free:

$$
\begin{array}{llllc}
\bar{\nabla} & \longmapsto & \nabla_{1} & \rightarrow & \mathbb{Z}\left[S_{1} \backslash S\right] \\
\downarrow \bar{\varphi} & & \downarrow_{\varphi_{1}} & & \downarrow h^{\prime} \\
\tilde{E} & \longmapsto & \tilde{E}_{1} & \rightarrow & E_{1} / E
\end{array} .
$$

We are going to show that

$$
\mathfrak{q}_{1}(\chi) / \mathfrak{q}(\chi)=(h)^{\frac{\left|S_{1} \backslash S\right|}{|G|} \chi(1)} .
$$

To that end we prove

(a) $\left[\left(\varphi_{1}\right)_{M}\right]-\left[\bar{\varphi}_{M}\right]-\left[h_{M}^{\prime}\right]=-\left[\operatorname{ker}\left(H^{1}(\tilde{E}) \rightarrow H^{1}\left(\tilde{E}_{1}\right)\right)\right]$,

(b) $\left[h_{M}^{\prime}\right]-\left[h_{M}\right]=-\left[\hat{G} \operatorname{Hom}_{\mathfrak{o}}(M, c l)\right]$,

(c) $\left[\bar{\varphi}_{M}\right]-\left[\varphi_{M}\right]=\left[\hat{G} \operatorname{Hom}_{\mathfrak{o}}(M, c l)\right]+\left[\operatorname{ker}\left(H^{-1}(\nabla) \rightarrow H^{-1}\left(\nabla_{1}\right)\right)\right]$

in $K_{0} T(\mathfrak{o})$. Adding up we obtain

$$
\begin{aligned}
& {\left[\left(\varphi_{1}\right)_{M}\right]-\left[\varphi_{M}\right]-\left[h_{M}\right]=} \\
& \quad-\left[\operatorname{ker}\left(H^{1}(\tilde{E}) \rightarrow H^{1}\left(\tilde{E}_{1}\right)\right)\right]+\left[\operatorname{ker}\left(H^{-1}(\nabla) \rightarrow H^{-1}\left(\nabla_{1}\right)\right)\right]=0
\end{aligned}
$$

on account of Theorem 2 (on adding $\mathbb{Z} G^{r}$, with identity map, to get $\tilde{E}, \tilde{A}, \tilde{E}_{1}, \tilde{A}_{1}$ ). Observe that $\operatorname{Hom}_{\mathfrak{o}}(M, C)$ is cohomologically trivial whenever $C$ is [Se, p.153]. 
Hence the formula stated above will follow from

(d) $\ell_{\mathfrak{o}}\left(\left[h_{M}\right]\right)=(h)^{\frac{\left|S_{1} \backslash S\right|}{|G|} \chi(1)}$

and it remains to show (a),(b),(c),(d).

For the proof of (a) apply $\operatorname{Hom}_{\mathfrak{o}}(M, \mathfrak{o} \otimes \cdot)$ to diagram (4) and take $G$-coinvariants and $G$-invariants. The vanishing of $H^{i}(C)$ for a cohomologically trivial $G$-module $C$ then yields

$$
\begin{array}{ccccc}
\operatorname{Hom}_{\mathfrak{o}}(M, \bar{\nabla})_{G} & \longmapsto & \operatorname{Hom}_{\mathfrak{o}}\left(M, \nabla_{1}\right)_{G} & \rightarrow & \operatorname{Hom}_{\mathfrak{o}}\left(M, \mathbb{Z}\left[S_{1} \backslash S\right]\right)_{G} \\
\downarrow \bar{\varphi}_{M} & & \downarrow\left(\varphi_{1}\right)_{M} & & \\
\operatorname{Hom}_{\mathfrak{o}}(M, \tilde{E})^{G} & \longmapsto & \operatorname{Hom}_{\mathfrak{o}}\left(M, \tilde{E}_{1}\right)^{G} & \rightarrow & \operatorname{ker}\left(\operatorname{Hom}_{\mathfrak{o}}\left(M, E_{1} / E\right)^{G} \rightarrow H^{1}(\tilde{E})\right)
\end{array}
$$

where $h^{\prime \prime}$ is induced by $h_{M}^{\prime}$. The snake lemma implies $\left[\left(\varphi_{1}\right)_{M}\right]-\left[\bar{\varphi}_{M}\right]=\left[h^{\prime \prime}\right]$. The short piece

$$
\operatorname{Hom}_{\mathfrak{o}}\left(M, \tilde{E}_{1}\right)^{G} \stackrel{e}{\rightarrow} \operatorname{Hom}_{\mathfrak{o}}\left(M, E_{1} / E\right)^{G \stackrel{d}{\rightarrow}} H^{1}(\tilde{E}) \stackrel{f}{\rightarrow} H^{1}\left(\tilde{E}_{1}\right)
$$

of the long cohomology sequence associated to $\tilde{E} \longmapsto \tilde{E}_{1} \rightarrow E_{1} / E$ gives $\operatorname{im}(e) \simeq$ $\operatorname{ker}(d)$ and $\operatorname{coker}(e) \simeq \operatorname{ker}(f) ;$ so $\left[\operatorname{coker}\left(h^{\prime \prime}\right)\right]=\left[\operatorname{coker}\left(h_{M}^{\prime}\right)\right]-[\operatorname{coker}(e)]=\left[\operatorname{coker}\left(h_{M}^{\prime}\right)\right]$ $-[\operatorname{ker}(f)]$ and $\left[h^{\prime \prime}\right]=\left[h_{M}^{\prime}\right]-\left[\operatorname{ker}\left(H^{1}(\tilde{E}) \rightarrow H^{1}\left(\tilde{E}_{1}\right)\right)\right]$.

Turning to (b) we apply diagram (2) in order to get

$$
\begin{array}{cccc}
\operatorname{Hom}_{\mathfrak{o}}\left(M, \mathbb{Z}\left[S_{1} \backslash S\right]\right)_{G} & = & \operatorname{Hom}_{\mathfrak{o}}\left(M, \mathbb{Z}\left[S_{1} \backslash S\right]\right)_{G} & \\
\downarrow h_{M}^{\prime} & & \downarrow h_{M} & \\
\operatorname{Hom}_{\mathfrak{o}}\left(M, E_{1} / E\right)^{G} & \longrightarrow & \operatorname{Hom}_{\mathfrak{o}}\left(M, \mathbb{Z}\left[S_{1} \backslash S\right]\right)^{G} & \left.\rightarrow \quad \operatorname{im}\left(\operatorname{Hom}_{\mathfrak{o}}\left(M, \mathbb{Z}\left[S_{1} \backslash S\right]\right)^{G} \rightarrow \operatorname{Hom}_{\mathfrak{o}}(M, c l)^{G}\right)\right)
\end{array}
$$

and so $\left[h_{M}^{\prime}\right]-\left[h_{M}\right]=-\left[\operatorname{im}\left(\operatorname{Hom}_{\mathfrak{o}}\left(M, \mathbb{Z}\left[S_{1} \backslash S\right]\right)^{G} \rightarrow \operatorname{Hom}_{\mathfrak{o}}(M, c l)^{G}\right)\right]$.

Because of $H^{0}\left(\mathbb{Z}\left[S_{1} \backslash S\right]\right)=0$ we have

$$
\operatorname{Hom}_{\mathfrak{o}}\left(M, \mathbb{Z}\left[S_{1} \backslash S\right]\right)^{G}=\hat{G} \operatorname{Hom}_{\mathfrak{o}}\left(M, \mathbb{Z}\left[S_{1} \backslash S\right]\right),
$$

so the surjectivity of $\operatorname{Hom}_{\mathfrak{o}}\left(M, \mathbb{Z}\left[S_{1} \backslash S\right]\right) \rightarrow \operatorname{Hom}_{\mathfrak{o}}(M, c l)$ implies

$$
\left[\operatorname{im}\left(\operatorname{Hom}_{\mathfrak{o}}\left(M, \mathbb{Z}\left[S_{1} \backslash S\right]\right)^{G} \rightarrow \operatorname{Hom}_{\mathfrak{o}}(M, c l)^{G}\right)\right]=\left[\hat{G} \operatorname{Hom}_{\mathfrak{o}}(M, c l)\right] .
$$

With respect to (c) we start from diagram (3) and obtain

$$
\begin{aligned}
& \frac{\operatorname{Hom}_{\mathfrak{o}}(M, c l)_{G}}{\operatorname{ker}\left(\operatorname{Hom}_{\mathfrak{o}}(M, c l)_{G} \rightarrow \operatorname{Hom}_{\mathfrak{o}}(M, \nabla)_{G}\right)} \longmapsto \operatorname{Hom}_{\mathfrak{o}}(M, \nabla)_{G} \rightarrow \operatorname{Hom}_{\mathfrak{o}}(M, \bar{\nabla})_{G} \\
& \downarrow \varphi_{M} \quad \downarrow \bar{\varphi}_{M} \\
& \operatorname{Hom}_{\mathfrak{o}}(M, \tilde{E})^{G}=\operatorname{Hom}_{\mathfrak{o}}(M, \tilde{E})^{G}
\end{aligned}
$$

and consequently

$$
\begin{aligned}
{\left[\bar{\varphi}_{M}\right]-\left[\varphi_{M}\right] } & =-\left[\operatorname{ker}\left(H^{-1}(c l) \rightarrow H^{-1}(\nabla)\right)\right]+\left[\operatorname{Hom}_{\mathfrak{o}}(M, c l)_{G}\right] \\
& =-\left[H^{-1}(c l)\right]+\left[\operatorname{ker}\left(H^{-1}(\nabla) \rightarrow H^{-1}(\bar{\nabla})\right)\right]+\left[\operatorname{Hom}_{\mathfrak{o}}(M, c l)_{G}\right] \\
& =\left[\operatorname{ker}\left(H^{-1}(\nabla) \rightarrow H^{-1}(\bar{\nabla})\right)\right]+\left[\hat{G} \operatorname{Hom}_{\mathfrak{o}}(M, c l)\right]
\end{aligned}
$$

by the cohomology sequence $H^{-1}(c l) \rightarrow H^{-1}(\nabla) \rightarrow H^{-1}(\bar{\nabla})$ induced by $c l \longmapsto \nabla \rightarrow$ $\bar{\nabla}$ and the definition of $H^{-1}(\mathrm{cl})$. By $(1), H^{-1}(\bar{\nabla})=H^{-1}\left(\nabla_{1}\right)$.

We are left with proving (d). The kernel of $h_{M}$ is $H^{-1}\left(\mathbb{Z}\left[S_{1} \backslash S\right]\right)=0$, and the cokernel is

$$
\begin{aligned}
\operatorname{Hom}_{\mathfrak{o}}\left(M, \mathbb{Z}\left[S_{1} \backslash S\right]\right)^{G} / h \hat{G} \operatorname{Hom}_{\mathfrak{o}}\left(M, \mathbb{Z}\left[S_{1} \backslash S\right]\right) \\
\quad=\operatorname{Hom}_{\mathfrak{o}}\left(M, \mathbb{Z}\left[S_{1} \backslash S\right]\right)^{G} / h \operatorname{Hom}_{\mathfrak{o}}\left(M, \mathbb{Z}\left[S_{1} \backslash S\right]\right)^{G}
\end{aligned}
$$


as $H^{0}\left(\mathbb{Z}\left[S_{1} \backslash S\right]\right)=0$. Because $\mathbb{Z}\left[S_{1} \backslash S\right]$ is $G$-free on $\frac{\left|S_{1} \backslash S\right|}{|G|}$ generators, the rank of the o-lattice $\operatorname{Hom}_{\mathfrak{o}}\left(M, \mathbb{Z}\left[S_{1} \backslash S\right]\right)^{G}$ is $\frac{\left|S_{1} \backslash S\right|}{|G|} \chi(1)$.

Turning now to $A$ it suffices to show

$$
A_{1}(\check{\chi}) / A(\check{\chi})=(-h)^{\left|\left(S_{1} \backslash S\right)_{*}\right| \chi(1)} .
$$

To that end we make use of

$$
c_{1}(\check{\chi}) / c(\check{\chi})=\prod_{\mathfrak{p} \in\left(S_{1} \backslash S\right)_{*}}(\log (N \mathfrak{p}))^{\chi(1)}
$$

which follows from Lemma 7 , because all primes $\mathfrak{p} \in S_{1} \backslash S$ have absolute degree 1 , hence $f_{\mathfrak{p}}=1, G_{\mathfrak{p}}=1, I_{\mathfrak{p}}=1$. Since $S_{1}^{\text {ram }}=S^{\text {ram }}$, this reduces to showing

$$
R_{1}(\check{\chi}) / R(\check{\chi})=\prod_{\mathfrak{p} \in\left(S_{1} \backslash S\right)_{*}}(-h \log (N \mathfrak{p}))^{\chi(1)}
$$

Choosing a $\tilde{\lambda}$ which makes the front face of the diagram

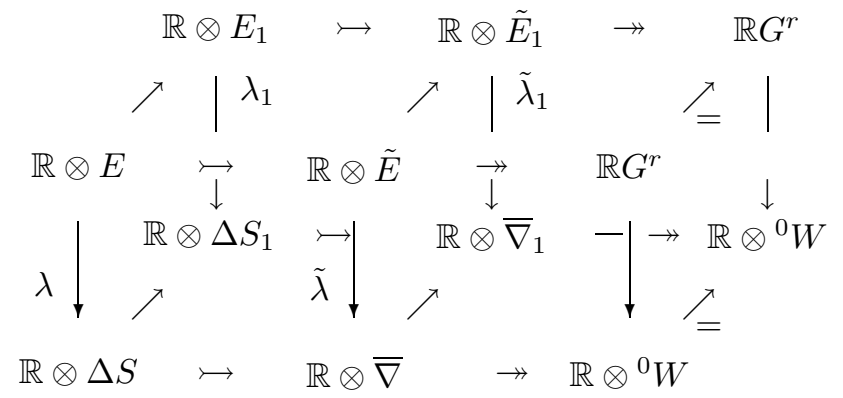

commute, we get a unique map $\tilde{\lambda}_{1}: \mathbb{R} \otimes \tilde{E}_{1} \rightarrow \mathbb{R} \otimes \bar{\nabla}_{1}$ which makes the whole diagram commute. We combine diagram (4), tensored with $\mathbb{R}$, with the middle face (and its cokernels) of the above diagram to obtain

$$
\begin{array}{ccccc}
\mathbb{R} \otimes \nabla & \longmapsto & \mathbb{R} \otimes \nabla_{1} & \rightarrow & \mathbb{R}\left[S_{1} \backslash S\right] \\
\downarrow \varphi & & \downarrow \varphi_{1} & & \downarrow h^{\prime} \\
\mathbb{R} \otimes \tilde{E} & \longmapsto & \mathbb{R} \otimes \tilde{E}_{1} & \rightarrow & \mathbb{R} \otimes E_{1} / E \\
\downarrow \tilde{\lambda} & & \downarrow \tilde{\lambda}_{1} & & \downarrow \\
\mathbb{R} \otimes \nabla & \longmapsto & \mathbb{R} \otimes \nabla_{1} & \rightarrow & \mathbb{R}\left[S_{1} \backslash S\right]
\end{array} .
$$

By diagram (2), tensored with $\mathbb{R}$, it follows that the right composite map is

$$
\mu: \mathfrak{p} \mapsto-h \log (N \mathfrak{p}) \cdot \mathfrak{p} .
$$

Applying $\operatorname{Hom}_{\mathbb{C} G}(V, \mathbb{C} \otimes \cdot)$ to the diagram we get

$$
R_{1}(\check{\chi}) / R(\check{\chi})=\operatorname{det}\left(\mu \mid \operatorname{Hom}_{\mathbb{C} G}\left(V, \mathbb{C}\left[S_{1} \backslash S\right]\right)\right) .
$$

From Frobenius reciprocity and the decomposition of $S_{1} \backslash S$ into $G$-orbits, all of which have length $|G|$, we obtain $\operatorname{Hom}_{\mathbb{C} G}\left(V, \mathbb{C}\left[S_{1} \backslash S\right]\right) \simeq \operatorname{Hom}_{\mathbb{C}}\left(V, \mathbb{C}\left[\left(S_{1} \backslash S\right)_{*}\right]\right)$. Since $\mu$ induces the diagonal matrix with diagonal entries $-h \log (N \mathfrak{p}), \mathfrak{p} \in\left(S_{1} \backslash S\right)_{*}$, on $\operatorname{Hom}_{\mathbb{C}}\left(V, \mathbb{C}\left[\left(S_{1} \backslash S\right)_{*}\right]\right)$, the assertion follows. 


\section{STEP 2}

This section is concerned with enlarging a set $S$ satisfying $c l_{S}=0$ to $S_{1}=$ $S_{\text {ram }}=S \cup S^{\mathrm{ram}}$.

From the end of $\S 2$ we recall

$$
{ }^{0} \Delta \nrightarrow \nabla \rightarrow \nabla_{1}
$$

by observing that $\bar{\nabla}=\nabla$ and $\Delta S_{1}=\nabla_{1}$. Since $S_{\text {ram }}=S_{1}$ and so $E_{\text {ram }}=E_{1}$, we get an auxiliary map $a$ as in the triangle preceding Lemma 5 , which we can and will assume to be already defined on the integral level. Namely, $\mathbb{Z} G^{r}$, with $r=r_{S}$, is free and $E_{\mathrm{ram}} \rightarrow \mathbb{Z}\left[S^{\mathrm{ram}}\right]$ is surjective, as follows from the triviality of the $S$-class group.

Hence

$$
\begin{aligned}
& \mathbb{Z} G^{r} \\
& { }^{a} \swarrow \quad \downarrow \\
& E \quad \longmapsto \quad E_{1} \quad \rightarrow \quad \mathbb{Z}\left[S^{\mathrm{ram}}\right]
\end{aligned}
$$

The triangle provides the short exact sequence

$$
\Delta \longmapsto \tilde{E} \rightarrow E_{1}
$$

where $\Delta=\bigoplus_{\mathfrak{p} \in S_{\star}^{\text {ram }}} \operatorname{ind}_{G_{\mathfrak{p}}}^{G} \Delta G_{\mathfrak{p}}$ is the kernel of its vertical map. Of course, $\tilde{E} \rightarrow E_{1}$ sends $(u, c) \in E \oplus \mathbb{Z} G^{r}$ to $u+a(c)$, hence $\Delta \rightarrow \tilde{E}$ sends $x$ to the pair $(-a(x), x) .^{2}$

We next build a commutative diagram

$$
\begin{array}{lllll}
{ }^{0} \Delta & \longrightarrow & \nabla & \rightarrow & \nabla_{1} \\
\downarrow \delta & & \downarrow & \\
{ }^{\prime} \varphi & & \downarrow \varphi_{1} \\
\Delta & \longrightarrow & \tilde{E} & \rightarrow & E_{1}
\end{array}
$$

in which the rows are (1) and (3), with isogenies $\varphi$ and $\varphi_{1}$, and with $\delta$ induced by local maps $\delta_{\mathfrak{p}}: \kappa_{\mathfrak{p}} \mapsto n\left(\left|G_{\mathfrak{p}}\right|-\hat{G}_{\mathfrak{p}}\right)$ for some integer $n \neq 0$, remembering $\Delta G_{\mathfrak{p}}^{0}=\mathbb{Z} G_{\mathfrak{p}} \cdot \kappa_{\mathfrak{p}}$ (see Proposition $1(\mathrm{a})$ ). Namely choosing $\varphi_{1}^{\prime}$ to be any isogeny and taking the $\delta$ with $n=1$, the diagram (4) tensored with $\mathbb{Q}$ can be completed by a $\varphi^{\prime}$ because of Maschke's theorem and then we get a diagram (4) by scaling by a suitable $n$.

We claim that

$$
\mathfrak{q}_{1}(\chi) / \mathfrak{q}(\chi)=\prod_{\mathfrak{p} \in S_{*}^{\text {ram }}} n^{\operatorname{dim} V^{G_{\mathfrak{p}}}-\operatorname{dim} V}\left|G_{\mathfrak{p}}\right|^{2 \operatorname{dim} V^{G_{\mathfrak{p}}}-\operatorname{dim} V} .
$$

In order to prove the claim we first proceed as in $\S 5$. From diagram (4) we get

$$
\begin{array}{rlrlc}
H^{-2}(\nabla) \rightarrow H^{-2}\left(\nabla_{1}\right) \rightarrow \operatorname{Hom}_{\mathfrak{o}}\left(M,^{0} \Delta\right)_{G} & \stackrel{e}{\rightarrow} & \operatorname{Hom}_{\mathfrak{o}}(M, \nabla)_{G} & \rightarrow & \operatorname{Hom}_{\mathfrak{o}}\left(M, \nabla_{1}\right)_{G} \\
\downarrow \delta_{M} & & \downarrow \varphi_{M} & & \downarrow\left(\varphi_{1}\right)_{M} \\
\operatorname{Hom}_{\mathfrak{o}}(M, \Delta)^{G} & \hookrightarrow & \operatorname{Hom}_{\mathfrak{o}}(M, \tilde{E})^{G} & \stackrel{f}{\rightarrow} & \operatorname{Hom}_{\mathfrak{o}}\left(M, E_{1}\right)^{G} \rightarrow H^{1}(\Delta)
\end{array}
$$

and so

- $\left[\varphi_{M}\right]-\left[\left(\varphi_{1}\right)_{M}\right]=-[\operatorname{coker}(f)]+\left[\delta_{M}\right]+[\operatorname{ker}(e)]$, since $\operatorname{im}\left(\left(\varphi_{1}\right)_{M}\right) \subset \operatorname{im}(f)$ and $\operatorname{ker}(e) \subset \operatorname{ker}\left(\delta_{M}\right)$,

- $\operatorname{coker}(f) \simeq \operatorname{coker}\left(H^{0}(\tilde{E}) \rightarrow H^{0}\left(E_{1}\right)\right)$ by the definition of $H^{0}$,

\footnotetext{
${ }^{2}$ Whenever it is convenient we will write units additively!
} 
- $\operatorname{ker}(e) \simeq \operatorname{coker}\left(H^{-2}(\nabla) \rightarrow H^{-2}\left(\nabla_{1}\right)\right)$, as follows from the top row in the diagram above,

- $\operatorname{ker}(e) \simeq \operatorname{coker}(f)$ because of Theorem 2 .

Collecting together we see that

$$
\left[\varphi_{M}\right]-\left[\left(\varphi_{1}\right)_{M}\right]=\left[\delta_{M}\right]
$$

In order to show

$$
\left[\delta_{M}\right]=\sum_{\mathfrak{p} \in S_{*}^{\mathrm{ram}}}\left[\left(\delta_{\mathfrak{p}}\right)_{M}\right]
$$

we use the following lemma, the proof of which is delayed to the end of the section.

Lemma 8. Let $M$ be an oG-lattice and $N$ an $\mathfrak{o} H$-module with $H$ a subgroup of $G$. Then $n \mapsto 1 \otimes n$ induces a map $\theta: \operatorname{Hom}_{\mathfrak{o}}(M, N) \rightarrow \operatorname{Hom}_{\mathfrak{o}}\left(M, \operatorname{ind}_{H}^{G} N\right)$ which induces an isomorphism $\bar{\theta}: \operatorname{Hom}_{\mathfrak{o}}(M, N)_{H} \rightarrow \operatorname{Hom}_{\mathfrak{o}}\left(M, \operatorname{ind}_{H}^{G} N\right)_{G}$.

From the lemma we obtain the commutative diagram

$$
\begin{aligned}
& \operatorname{Hom}_{\mathfrak{o}}\left(M, \Delta G_{\mathfrak{p}}^{0}\right)_{G_{\mathfrak{p}}} \quad \stackrel{\hat{G}_{\mathfrak{p}}}{\rightarrow} \quad \operatorname{Hom}_{\mathfrak{o}}\left(M, \Delta G_{\mathfrak{p}}^{0}\right)^{G_{\mathfrak{p}}} \quad \stackrel{\delta_{\mathfrak{p}}}{\rightarrow} \quad \operatorname{Hom}_{\mathfrak{o}}\left(M, \Delta G_{\mathfrak{p}}\right)^{G_{\mathfrak{p}}} \\
& \simeq \downarrow \bar{\theta} \quad \simeq \downarrow \theta^{\prime} \quad \simeq \downarrow \theta^{\prime} \\
& \operatorname{Hom}_{\mathfrak{o}}\left(M, \operatorname{ind}_{G_{\mathfrak{p}}}^{G} \Delta G_{\mathfrak{p}}^{0}\right)_{G} \quad \stackrel{\hat{G}}{\rightarrow} \operatorname{Hom}_{\mathfrak{o}}\left(M, \operatorname{ind}_{G_{\mathfrak{p}}}^{G} \Delta G_{\mathfrak{p}}^{0}\right)^{G} \stackrel{\operatorname{ind} \delta_{\mathfrak{p}}}{\rightarrow} \operatorname{Hom}_{\mathfrak{o}}\left(M, \operatorname{ind}_{G_{\mathfrak{p}}}^{G} \Delta G_{\mathfrak{p}}\right)^{G}
\end{aligned}
$$

and thus (b). Here $\theta^{\prime}$, the map of Frobenius reciprocity, sends $s$ to $\tilde{s}$ where $\tilde{s}(m)=$ $\sum_{g} g \otimes s\left(g^{-1} m\right)$ and $g$ runs through a set of coset representatives of $G_{\mathfrak{p}}$ in $G$.

So our formula for the quotient of the $q$-indices follows from

$$
\ell_{\mathfrak{o}}\left(\left[\left(\delta_{\mathfrak{p}}\right)_{M}\right]\right)=n^{\operatorname{dim} V-\operatorname{dim} V^{G_{\mathfrak{p}}}}\left|G_{\mathfrak{p}}\right|^{\operatorname{dim} V-2 \operatorname{dim} V^{G_{\mathfrak{p}}} .}
$$

We prove this whenever $M$ is an $\mathfrak{o} G_{\mathfrak{p}}$-lattice spanning the $F G_{\mathfrak{p}}$-space $V$.

Since $\delta_{\mathfrak{p}}$ is an isogeny between the two ends of the "Tate-sequence" $\Delta G_{\mathfrak{p}} \longmapsto$ $\mathbb{Z} G_{\mathfrak{p}} \rightarrow \mathbb{Z} G_{\mathfrak{p}} \rightarrow \Delta G_{\mathfrak{p}}^{0}$ that is obtained from $\Delta G_{\mathfrak{p}} \longmapsto \mathbb{Z} G_{\mathfrak{p}} \rightarrow \mathbb{Z}$ and its dual, we are allowed to replace $M$ by any $\mathfrak{o} G_{\mathfrak{p}}$-lattice affording $\chi$ [Ta2, Lemme 7, p.60].

In the computations to follow we may as well drop the index $\mathfrak{p}$ and assume that $\chi$ is irreducible. Moreover, we may assume that $n=1$. Denoting, temporarily, our $\delta$ by $\delta(n)$ we have $\delta(n)=n \cdot \delta(1)$. Hence

$$
\left[\delta(n)_{M}\right]=\left[\delta(1)_{M}\right]+\left[\operatorname{coker}\left(\operatorname{Hom}_{\mathfrak{o}}(M, \Delta)^{G} \stackrel{n}{\rightarrow} \operatorname{Hom}_{\mathfrak{o}}(M, \Delta)^{G}\right)\right] .
$$

The second summand has length $n^{(\chi, \Delta G)}=n^{\operatorname{dim} V-\operatorname{dim} V^{G}}$.

If $\chi$ is trivial, we are in the situation $\mathfrak{o}=\mathbb{Z}, M=\mathbb{Z}$ and, observing $\left(\Delta G^{0}\right)^{G}=$ $0=(\Delta G)^{G}$, we have

$$
\ell_{\mathfrak{o}}\left(\delta_{\mathbb{Z}}\right)=1 / \ell_{\mathfrak{o}}\left(\left(\Delta G^{0}\right)_{G}\right)=\ell_{\mathfrak{o}}\left(H^{-1}\left(G, \Delta G^{0}\right)\right)^{-1}=\ell_{\mathfrak{o}}\left(H^{0}(G, \mathbb{Z})\right)^{-1}=|G|^{-1} \mathfrak{o} .
$$

If $\chi$ is non-trivial, we employ the commutative diagram

$$
\begin{array}{rccccc}
\Delta G^{0} & \longmapsto & \mathbb{Z} G & \rightarrow & \overline{\mathbb{Z} G} & \\
\downarrow \delta & & \| & & \downarrow & \text { with } \kappa \mapsto|G|-\hat{G}, \overline{\mathbb{Z} G}=\mathbb{Z} G /(|G|-\hat{G}) \text { and } \\
\Delta G & \longmapsto & \mathbb{Z} G & \rightarrow & \mathbb{Z} & \text { with the augmentation on the right hand side. }
\end{array}
$$


We obtain

$$
\begin{aligned}
& H^{-2}(\overline{\mathbb{Z} G}) \longmapsto \operatorname{Hom}_{\mathfrak{o}}\left(M, \Delta G^{0}\right)_{G} \quad \rightarrow \quad \operatorname{Hom}_{\mathfrak{o}}(M, \mathbb{Z} G)_{G} \quad \rightarrow \quad \operatorname{Hom}_{\mathfrak{o}}(M, \overline{\mathbb{Z} G})_{G} \\
& \downarrow \delta_{M} \quad \downarrow \hat{G} \rightarrow \downarrow \\
& \operatorname{Hom}_{\mathfrak{o}}(M, \Delta G)^{G} \longmapsto \operatorname{Hom}_{\mathfrak{o}}(M, \mathbb{Z} G)^{G} \rightarrow \operatorname{Hom}_{\mathfrak{o}}(M, \mathbb{Z})^{G} \rightarrow H^{1}(\Delta G) .
\end{aligned}
$$

As $\chi \neq 1, \operatorname{Hom}_{\mathfrak{o}}(M, \mathbb{Z})^{G}=0$ and so $H^{-2}(\overline{\mathbb{Z} G})=H^{-1}\left(\Delta G^{0}\right)=H^{0}(\mathbb{Z})=0$. Also, the middle $\hat{G}$ is an isomorphism, since $\mathbb{Z} G$ is free. Hence $\left[\delta_{M}\right]=\left[\operatorname{Hom}_{\mathfrak{o}}(M, \overline{\mathbb{Z} G})_{G}\right]$. The length of this has been computed in [Ch1]; see also [We, $\S 13$, after the Claim in Step 1 of the proof of Theorem 8]:

$$
\begin{aligned}
& \quad \ell_{\mathfrak{o}}\left(\operatorname{Hom}_{\mathfrak{o}}(M, \overline{\mathbb{Z} G})_{G}\right) \\
& \quad=\ell_{\mathfrak{o}}\left(\operatorname{coker}\left(\operatorname{Hom}_{\mathfrak{o}}(M, \mathbb{Z} G)^{G} \stackrel{|G|}{\longrightarrow} \operatorname{Hom}_{\mathfrak{o}}(M, \mathbb{Z} G)^{G}\right)\right)=|G|^{\operatorname{dim} \check{V}} \mathfrak{o} .
\end{aligned}
$$

We now pass to $A$ and show that also

$$
A_{1}(\check{\chi}) / A(\check{\chi})=\prod_{\mathfrak{p} \in S_{*}^{\text {ram }}} n^{\operatorname{dim} V^{G_{\mathfrak{p}}}-\operatorname{dim} V}\left|G_{\mathfrak{p}}\right|^{2 \operatorname{dim} V^{G_{\mathfrak{p}}}-\operatorname{dim} V} .
$$

For that we recall from Lemma 7 that

$$
c_{1}(\check{\chi}) / c(\check{\chi})=\prod_{\mathfrak{p} \in S_{*}^{\text {ram }}}\left(\frac{\log (N \mathfrak{p})}{f_{\mathfrak{p}}}\right)^{\operatorname{dim} \check{V}^{G_{\mathfrak{p}}}} \operatorname{det}\left(1-\phi_{\mathfrak{p}} \mid \check{V}^{I_{\mathfrak{p}}} / \check{V}^{G_{\mathfrak{p}}}\right),
$$

and so

$$
\begin{aligned}
A_{1}(\check{\chi}) / A(\check{\chi})= & R_{1}(\check{\chi}) c(\check{\chi}) \prod_{\mathfrak{p} \in S_{*}^{\text {ram }}}\left(e_{\mathfrak{p}} \log (N \mathfrak{p})\right)^{\check{\chi}(1)} R(\check{\chi})^{-1} c_{1}(\check{\chi})^{-1} \\
= & \frac{R_{1}(\check{\chi})}{R(\check{\chi})} \prod_{\mathfrak{p} \in S_{*}^{\text {ram }}}\left(e_{\mathfrak{p}} \log (N \mathfrak{p})\right)^{\operatorname{dim} \check{V}} f_{\mathfrak{p}}^{\operatorname{dim} \check{V}^{G_{\mathfrak{p}}}}(\log (N \mathfrak{p}))^{-\operatorname{dim} \check{V}^{G_{\mathfrak{p}}}} \\
& \times \operatorname{det}\left(1-\phi_{\mathfrak{p}} \mid \check{V}^{I_{\mathfrak{p}}} / \check{V}^{G_{\mathfrak{p}}}\right)^{-1} .
\end{aligned}
$$

In order to compute the quotient of the regulators stack diagram (4), tensored with $\mathbb{R}$, on top of

$$
\begin{array}{cccccc}
\mathbb{R} \otimes \Delta & \longmapsto & \mathbb{R} \otimes \tilde{E} & \rightarrow & \mathbb{R} \otimes E_{1} \\
\downarrow d & & \downarrow \tilde{\lambda} & & \downarrow \lambda_{1} \\
\mathbb{R} \otimes{ }^{0} \Delta & \longmapsto & \mathbb{R} \otimes \nabla & \rightarrow & \mathbb{R} \otimes \nabla_{1}
\end{array},
$$

the rows of which are (3) and (1). The left vertical map $d$ is defined by the diagram. It follows readily that $R_{1}(\check{\chi}) / R(\check{\chi})=\operatorname{det}\left(d \delta \mid \operatorname{Hom}_{\mathbb{C} G}\left(V, \mathbb{C} \otimes{ }^{0} \Delta\right)\right)^{-1}$. By (4), $d \delta$ sends a generator $1 \otimes \kappa_{\mathfrak{p}} \in \mathbb{R} \otimes \Delta G_{\mathfrak{p}}^{0}$ to $d\left(1 \otimes n\left(\left|G_{\mathfrak{p}}\right|-\hat{G}_{\mathfrak{p}}\right)\right)$. To compute this we view it as an element of $\mathbb{R} \otimes \nabla$, by means of (5), which, by (3), equals $\tilde{\lambda}\left(-a\left(1 \otimes n\left(\left|G_{\mathfrak{p}}\right|-\hat{G}_{\mathfrak{p}}\right)\right), 1 \otimes n\left(\left|G_{\mathfrak{p}}\right|-\hat{G}_{\mathfrak{p}}\right)\right)$ which, in turn, by Lemma 5 is

$$
\begin{aligned}
\lambda\left(-a\left(1 \otimes n\left(\left|G_{\mathfrak{p}}\right|-\hat{G}_{\mathfrak{p}}\right)\right)\right)+n\left(\left|G_{\mathfrak{p}}\right|-\hat{G_{\mathfrak{p}}}\right) \tilde{\lambda}\left(1 \otimes 1_{\mathfrak{p}}\right) \\
=-\sum_{\mathfrak{p}^{\prime} \in S} \log \left|a\left(n\left(\left|G_{\mathfrak{p}}\right|-\hat{G}_{\mathfrak{p}}\right)\right)\right|_{\mathfrak{p}^{\prime}} \otimes \mathfrak{p}^{\prime} \\
\quad+n\left(\left|G_{\mathfrak{p}}\right|-\hat{G}_{\mathfrak{p}}\right)\left(-\log (N \mathfrak{p}) \otimes \rho_{\mathfrak{p}}+\sum_{\mathfrak{p}^{\prime} \in S} \log \left|a\left(1_{\mathfrak{p}}\right)\right|_{\mathfrak{p}^{\prime}} \otimes \mathfrak{p}^{\prime}\right) \\
=-n \log (N \mathfrak{p}) \otimes\left(\left|G_{\mathfrak{p}}\right|-\hat{G}_{\mathfrak{p}}\right) \rho_{\mathfrak{p}}=-n \log (N \mathfrak{p}) \otimes y_{\mathfrak{p}} \kappa_{\mathfrak{p}},
\end{aligned}
$$


by Proposition 1(b). It follows from Frobenius reciprocity that under the identification

$$
\operatorname{Hom}_{\mathbb{C} G}\left(V, \mathbb{C} \otimes{ }^{0} \Delta\right)=\bigoplus_{\mathfrak{p} \in S_{*}^{\text {ram }}} \operatorname{Hom}_{\mathbb{C} G_{\mathfrak{p}}}\left(V, \Delta G_{\mathfrak{p}}^{0}\right)
$$

the map $d \delta$ corresponds to $\bigoplus_{\mathfrak{p} \in S_{*}^{\text {ram }}}\left(-n \log (N \mathfrak{p})\left(y_{\mathfrak{p}}\right)_{\check{V}}\right)$, in the notation of Proposition $1(\mathrm{c})$.

Thus

$$
\begin{aligned}
R_{1}(\check{\chi}) / R(\check{\chi}) & =\prod_{\mathfrak{p} \in S_{*}^{\text {ram }}}(-n \log (N \mathfrak{p}))^{-\left(\chi, \Delta G_{\mathfrak{p}}^{0}\right)} \operatorname{det}\left(\left(y_{\mathfrak{p}}\right)_{\check{V}}\right)^{-1} \\
& =\prod_{\mathfrak{p} \in S_{*}^{\text {ram }}}(-n \log (N \mathfrak{p}))^{\operatorname{dim} \check{V}^{G \mathfrak{p}}-\operatorname{dim} \check{V}} \operatorname{det}\left(\left(y_{\mathfrak{p}}\right)_{\check{V}}\right)^{-1},
\end{aligned}
$$

as $\left(\chi, \Delta G_{\mathfrak{p}}^{0}\right)=\left(\check{\chi}, \Delta G_{\mathfrak{p}}\right)=\operatorname{dim} \check{V}-\operatorname{dim} \check{V}^{G_{\mathfrak{p}}}$. By combining this with Proposition 1(c), we obtain from (d), with the abbreviation $z=\operatorname{dim} \check{V}^{G_{\mathfrak{p}}}-\operatorname{dim} \check{V}$,

$$
\begin{aligned}
& A_{1}(\check{\chi}) / A(\check{\chi})=\prod n^{z}(\log (N \mathfrak{p}))^{z} \operatorname{det}\left(1-\phi_{\mathfrak{p}}\right) e_{\mathfrak{p}}^{z}\left|G_{\mathfrak{p}}\right|^{z} e_{\mathfrak{p}}^{\operatorname{dim} \check{V}}(\log (N \mathfrak{p}))^{\operatorname{dim} \check{V}} \\
& \times\left|G_{\mathfrak{p}}\right|^{\operatorname{dim} \check{V}^{G \mathfrak{p}}} e_{\mathfrak{p}}^{-\operatorname{dim} \check{V}^{G \mathfrak{p}}}(\log (N \mathfrak{p}))^{-\operatorname{dim} \check{V}^{G \mathfrak{p}}} \operatorname{det}\left(1-\phi_{\mathfrak{p}}\right)^{-1} \\
& =\prod_{\mathfrak{p}} n^{\operatorname{dim} \check{V}^{G_{\mathfrak{p}}}-\operatorname{dim} \check{V}}\left|G_{\mathfrak{p}}\right|^{2 \operatorname{dim} \check{V}^{G_{\mathfrak{p}}}-\operatorname{dim} \check{V}} .
\end{aligned}
$$

Proof of Lemma 8. Clearly $\bar{\theta}$ is surjective. Moreover, since $\theta^{\prime}$ in the commutative diagram

$$
\begin{array}{ccc}
\operatorname{Hom}_{\mathfrak{o}}(M, N)_{H} & \stackrel{\bar{\theta}}{\longrightarrow} & \operatorname{Hom}_{\mathfrak{o}}\left(M, \operatorname{ind}_{H}^{G} N\right)_{G} \\
\downarrow \hat{H} & & \downarrow \hat{G} \\
\operatorname{Hom}_{\mathfrak{o}}(M, N)^{H} & \stackrel{\theta^{\prime}}{\longrightarrow} & \operatorname{Hom}_{\mathfrak{o}}\left(M, \operatorname{ind}_{H}^{G} N\right)^{G}
\end{array}
$$

is an isomorphism, we have

$$
\operatorname{ker}(\bar{\theta}) \longmapsto H^{-1}\left(H, \operatorname{Hom}_{\mathfrak{o}}(M, N)\right) \rightarrow H^{-1}\left(G, \operatorname{Hom}_{\mathfrak{o}}\left(M, \operatorname{ind}_{H}^{G} N\right)\right) .
$$

Thus $\operatorname{ker}(\bar{\theta})=0$ if, and only if, the map on $H^{-1}$ is an isomorphism. Since $M$ is an o $G$-lattice, this assertion can be shifted to dimension 0 .

\section{STEP 3}

In this section we start out from a set $S$ satisfying $S=S_{\text {ram }}$ and $c l_{S}=0$, and let $S_{1}=S \cup G \mathfrak{p}_{1}$ for a prime $\mathfrak{p}_{1}$ of $K$.

We are going to compare $q$-indices and $A$-numbers by means of the commutative diagram

$$
\begin{array}{lllll}
\nabla & \longmapsto & \nabla_{1} & \rightarrow & \mathbb{Z}\left[G \mathfrak{p}_{1}\right] \\
\downarrow \varphi & & \downarrow \varphi_{1} & & \downarrow n \\
E & \longmapsto & E_{1} & \rightarrow & \mathbb{Z}\left[G \mathfrak{p}_{1}\right]
\end{array}
$$

in which the upper row is induced by $\mathbb{Z}[S] \nrightarrow \mathbb{Z}\left[S_{1}\right] \rightarrow \mathbb{Z}\left[G \mathfrak{p}_{1}\right]$, on taking $\nabla=$ $\Delta S, \nabla_{1}=\Delta S_{1}$ into account, and the lower row by $u_{1} \mapsto \sum_{g} \bmod G_{\mathfrak{p}_{1}} v_{g_{\mathfrak{p}}}\left(u_{1}\right) g \mathfrak{p}_{1}$ for $u_{1} \in E_{1}$.

Moreover, we have chosen an isogeny $\varphi^{\prime}: \nabla \rightarrow E$ and the identity map on $\mathbb{Q}\left[G \mathfrak{p}_{1}\right]$ in order to get a $\varphi_{1}^{\prime}: \mathbb{Q} \otimes \nabla_{1} \rightarrow \mathbb{Q} \otimes E_{1}$, making the diagram commute on the $\mathbb{Q}$-level, and obtain $\varphi, \varphi_{1}, n$ by scaling with an appropriate $0 \neq n \in \mathbb{Z}$. 
We first show that

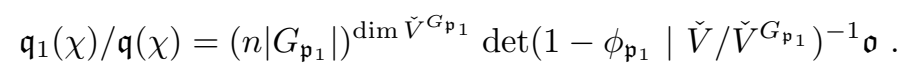

Namely, from the diagram above we obtain

$$
\begin{array}{rlccc}
H^{-2}\left(\mathbb{Z}\left[G \mathfrak{p}_{1}\right]\right) \stackrel{\delta_{-2}}{\longrightarrow} \operatorname{Hom}_{\mathfrak{o}}(M, \nabla)_{G} & \rightarrow & \operatorname{Hom}_{\mathfrak{v}}\left(M, \nabla_{1}\right)_{G} & \rightarrow & \operatorname{Hom}_{\mathfrak{o}}\left(M, \mathbb{Z}\left[G \mathfrak{p}_{1}\right]\right)_{G} \\
\downarrow \varphi_{M} & & \downarrow\left(\varphi_{1}\right)_{M} & & \downarrow n_{M} \\
\operatorname{Hom}_{\mathfrak{o}}(M, E)^{G} & \longrightarrow & \operatorname{Hom}_{\mathfrak{o}}\left(M, E_{1}\right)^{G} & \rightarrow & \operatorname{Hom}_{\mathfrak{o}}\left(M, \mathbb{Z}\left[G \mathfrak{p}_{1}\right]\right)^{G} \rightarrow H^{1}(E) \rightarrow H^{1}\left(E_{1}\right)
\end{array}
$$

and so

$$
\begin{aligned}
{\left[\left(\varphi_{1}\right)_{M}\right]-\left[\varphi_{M}\right] } & =\left[\operatorname{im}\left(\delta_{-2}\right)\right]+\left[n_{M}\right]-\left[\operatorname{ker}\left(H^{1}(E) \rightarrow H^{1}\left(E_{1}\right)\right)\right] \\
& =\left[\operatorname{ker}\left(H^{-1}(\nabla) \rightarrow H^{-1}\left(\nabla_{1}\right)\right)\right]+\left[n_{M}\right]-\left[\operatorname{ker}\left(H^{1}(E) \rightarrow H^{1}\left(E_{1}\right)\right)\right] \\
& =\left[n_{M}\right],
\end{aligned}
$$

by Theorem 2. From the definition of the map $n_{M}$ and $\mathbb{Z}\left[G \mathfrak{p}_{1}\right]=\operatorname{ind}_{G_{\mathfrak{p}_{1}}}^{G} \mathbb{Z}$ we get

$$
\begin{aligned}
{\left[n_{M}\right] } & =\left[H^{0}\left(\mathbb{Z}\left[G \mathfrak{p}_{1}\right]\right)\right]-\left[H^{-1}\left(\mathbb{Z}\left[G \mathfrak{p}_{1}\right]\right)\right]+\left[\operatorname{coker}\left(n \mid \operatorname{Hom}_{\mathfrak{o} G}\left(M, \operatorname{ind}_{G_{\mathfrak{p}_{1}}}^{G} \mathbb{Z}\right)\right)\right] \\
& =\left[H^{0}\left(G_{\mathfrak{p}_{1}}, \check{M}\right)\right]-\left[H^{-1}\left(G_{\mathfrak{p}_{1}}, \check{M}\right)\right]+\left[\operatorname{coker}\left(n \mid \check{M}^{G_{\mathfrak{p}_{1}}}\right)\right]
\end{aligned}
$$

by Shapiro's lemma with $\check{M}=\operatorname{Hom}_{\mathfrak{o}}(M, \mathfrak{o})$ and by Frobenius reciprocity. Taking lengths and noting that $G_{\mathfrak{p}_{1}}$ is cyclic we get

$$
\begin{aligned}
\mathfrak{q}_{1}(\chi) / \mathfrak{q}(\chi) & =\left(\text { Herbrand quotient of } G_{\mathfrak{p}_{1}} \text { acting on } \check{M}\right) \times(n)^{\operatorname{dim} \check{V}^{G_{\mathfrak{p}_{1}}}} \\
& =\left(n\left|G_{\mathfrak{p}_{1}}\right|\right)^{\operatorname{dim} \check{V}^{G \mathfrak{p}_{1}}} \operatorname{det}\left(1-\phi_{\mathfrak{p}_{1}} \mid \check{V} / \check{V}^{G_{\mathfrak{p}_{1}}}\right)^{-1} \mathfrak{o} .
\end{aligned}
$$

The Herbrand quotient part of this is additive in $\check{\chi}$, hence can be checked on irreducible characters of $G_{\mathfrak{p}_{1}}$ [We, Proposition 8].

Turning finally to $A$ it suffices to show that

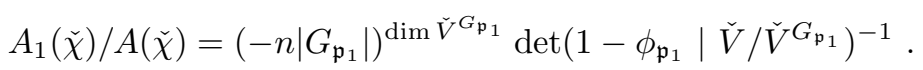

Because of Lemma 7 this reduces to proving

$$
R_{1}(\check{\chi}) / R(\check{\chi})=\left(-n \log \left(N \mathfrak{p}_{1}\right)\right)^{\operatorname{dim} \check{V}^{G_{\mathfrak{p}_{1}}}},
$$

which directly follows by combining the commutative diagram

$$
\begin{aligned}
& \mathbb{C} \otimes E \quad \longmapsto \quad \mathbb{C} \otimes E_{1} \quad \rightarrow \quad \quad \quad \mathbb{C}\left[G \mathfrak{p}_{1}\right] \\
& \downarrow \lambda \quad \downarrow \lambda_{1} \quad \downarrow-\log \left(N \mathfrak{p}_{1}\right) \\
& \mathbb{C} \otimes \nabla \quad \longmapsto \mathbb{C} \otimes \nabla_{1} \quad \rightarrow \quad \quad \quad \mathbb{C}\left[G \mathfrak{p}_{1}\right]
\end{aligned}
$$

with the diagram at the beginning of this section and applying $\operatorname{Hom}_{\mathbb{C} G}(V, \cdot)$.

\section{General Reductions}

For the purpose of stating the following proposition and its corollary we write $\mathfrak{a}_{K / k}(\chi)$ for the ideal $\mathfrak{a}(\chi)$ in Theorem B.

Proposition 9. (a) $\mathfrak{a}_{K / k}$ is additive, i.e. $\mathfrak{a}_{K / k}\left(\chi_{1}+\chi_{2}\right)=\mathfrak{a}_{K / k}\left(\chi_{1}\right) \mathfrak{a}_{K / k}\left(\chi_{2}\right)$ for characters $\chi_{1}, \chi_{2}$ of $G$.

(b) $\mathfrak{a}_{K / k}(1)=(1)$.

(c) Let $G^{\prime}$ be a subgroup of $G$ with fixed field $K^{\prime}$. Then for any character $\chi^{\prime}$ of $G^{\prime}$ we have $\mathfrak{a}_{K / K^{\prime}}\left(\chi^{\prime}\right)=\mathfrak{a}_{K / k}\left(\operatorname{ind}_{G^{\prime}}^{G} \chi^{\prime}\right)$.

(d) If $G^{\prime}$ is normal in $G$ and $\bar{\chi}$ a character of $\bar{G}=G / G^{\prime}$, then $\mathfrak{a}_{K^{\prime} / k}(\bar{\chi})=$ $\mathfrak{a}_{K / k}\left(\operatorname{infl} \frac{G}{G} \bar{\chi}\right)$. 
(a), (b), and (c) follow from [Ta2, pp.31,60] with $\mathfrak{a}$ replaced by $\mathfrak{a}_{S}$ for a large set $S$; (d) has been observed in [Ch1] in the form $\mathfrak{a}_{\varphi^{\prime}}(\bar{\chi})=\mathfrak{a}_{\varphi}(\operatorname{infl} G \bar{G} \bar{\chi})$ for a given isogeny $\Delta S \stackrel{\varphi}{\rightarrow} E$ and a derived $\varphi^{\prime}: \Delta S^{\prime} \rightarrow E^{\prime}$, where $S^{\prime}$ is the set of all primes of $K^{\prime}$ which lie below the ones in a sufficiently large set $S$. For details see [We, §11]. So Proposition 9 is a direct consequence of Theorem B, since $\mathfrak{a}$ is independent of $S$ and the special choice of an isogeny.

Corollary. $\mathfrak{a}_{K / k}(\chi)$ is determined by the $\mathfrak{a}_{K^{\prime} / k^{\prime}}\left(\chi^{\prime}\right)$, where $K^{\prime} / k^{\prime}$ runs through the cyclic subextensions of $K / k$ and $\chi^{\prime}$ through the corresponding characters.

This is merely the above proposition combined with the Brauer induction theorem by which we can write a given $\chi$ as a $\mathbb{Z}$-linear combination of characters induced from linear characters of subgroups $G^{\prime}$ of $G$.

We next start out from a given embedding $\underline{\varphi}: \Delta S \rightarrow E$ and describe a procedure by means of which we obtain an isogeny $\varphi: \nabla \rightarrow \tilde{E}=E \oplus \mathbb{Z} G^{r}, r=r_{S}$. Our ultimate goal in this section is to give $\mathfrak{a}(\chi)$ in terms of $\underline{\varphi}$.

We begin by choosing and fixing an infinite prime, say $\infty$, in $S_{*}$ and defining

firstly, $\sigma_{\infty}: \mathbb{Z} S \rightarrow \Delta S \oplus \mathbb{Z}$ by $\mathfrak{p} \mapsto(|G| \mathfrak{p}-\hat{G} \infty, 1)$

and secondly, for $\mathfrak{p} \in S_{*}^{\text {ram }}$,

$\sigma_{\mathfrak{p}}: W_{\mathfrak{p}}^{0} \rightarrow \Delta G_{\mathfrak{p}}^{0} \oplus \mathbb{Z}$ by $W_{\mathfrak{p}}^{0} \ni \nu \mapsto\left(\left|G_{\mathfrak{p}}\right| \nu-\iota_{\mathfrak{p}}(\nu) \hat{G}_{\mathfrak{p}} \eta_{\mathfrak{p}}, \iota_{\mathfrak{p}}(\nu)\right)$ where $\iota_{\mathfrak{p}}$ is the inertial augmentation at $\mathfrak{p}$ and $\eta_{\mathfrak{p}}$ is as in Proposition 1(a),

$\delta_{\mathfrak{p}}: \Delta G_{\mathfrak{p}}^{0} \rightarrow \Delta G_{\mathfrak{p}}$ by $\kappa_{\mathfrak{p}} \mapsto\left|G_{\mathfrak{p}}\right|-\hat{G}_{\mathfrak{p}}$.

These are $\mathbb{Z} G$-maps, respectively $\mathbb{Z} G_{\mathfrak{p}}$-maps, which fit into the commutative diagrams

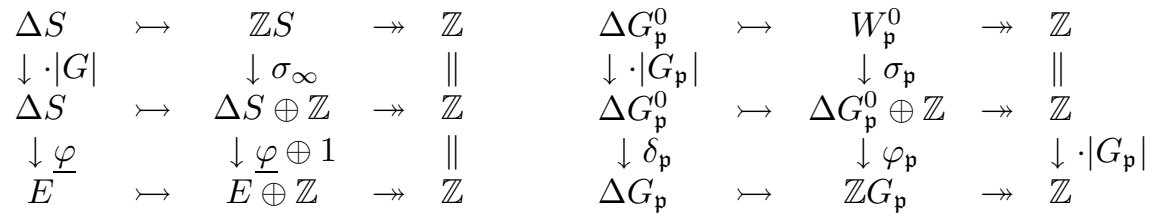

where $\varphi_{\mathfrak{p}}$ abbreviates the composite of the map $\delta_{\mathfrak{p}} \oplus 1$ and the map $\Delta G_{\mathfrak{p}} \oplus \mathbb{Z} \rightarrow \mathbb{Z} G_{\mathfrak{p}}$ given by $(x, z) \mapsto x+z \hat{G}_{\mathfrak{p}}$. We combine these maps and arrive at the map $\varphi_{+}$in the following diagram:

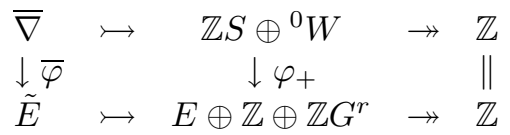

On $\mathbb{Z} S$ this map is determined by the left diagram above; on ${ }^{0} W$ it is

$$
\left(0, \sum_{\mathfrak{p} \in S_{*}^{\text {ram }}} \operatorname{ind}_{G_{\mathfrak{p}}}^{G} \iota_{\mathfrak{p}}, \sum_{\mathfrak{p} \in S_{*}^{\text {ram }}} \operatorname{ind}_{G_{\mathfrak{p}}}^{G}\left(\varphi_{\mathfrak{p}} \sigma_{\mathfrak{p}}\right)\right) .
$$

The lower row maps $(u, z, x)$ to $z$.

Definition. The isogeny $\varphi: \nabla \rightarrow \tilde{E}$ associated to the given $\underline{\varphi}: \Delta S \rightarrow E$ is the composite map $\nabla \rightarrow \bar{\nabla} \stackrel{\bar{\varphi}}{\rightarrow} \tilde{E}$, where $\bar{\varphi}$ is defined by the above diagram. 
Proposition 10. Let $G$ be abelian and define $\mathfrak{o}^{\prime}=\mathfrak{o}\left[\frac{1}{|G|}\right]$. If $\chi$ is an irreducible character of $G$ with representation space $V$, then

$$
\mathfrak{a}(\chi) \cdot \mathfrak{o}^{\prime}=\frac{\operatorname{det}\left(\lambda \underline{\varphi} \mid \operatorname{Hom}_{\mathbb{C} G}(V, \mathbb{C} \otimes \Delta S)\right)}{c_{S}(\check{\chi})} \cdot \frac{\ell_{\mathfrak{o}^{\prime}}\left(\left(\mathfrak{o}^{\prime} \otimes c l\right)^{\chi}\right)}{\ell_{\mathfrak{o}^{\prime}}\left(\left(\mathfrak{o}^{\prime} \otimes \operatorname{coker}(\underline{\varphi})\right)^{\chi}\right)}
$$

where cl is the $S$-class group of $K$ and $X^{\chi}=e_{\chi} X$ is the $\chi$-eigenspace of the $\mathfrak{o}^{\prime} G$ module $X$.

The proof of the proposition splits into two parts, one being concerned with $\mathfrak{q}$ and the other with $A$. We first turn to $\mathfrak{q}_{\varphi}(\chi)$, where $\chi$ is a linear character of the abelian group $G$, and let $M=\mathfrak{o}^{\prime} G e_{\chi}=\mathfrak{o}^{\prime} e_{\chi}$ be the $\mathfrak{o}^{\prime} G$-lattice affording $\chi$, where $e_{\chi}=\frac{1}{|G|} \sum_{g \in G} \chi\left(g^{-1}\right) g$ is the primitive idempotent belonging to $\chi$.

Claim. $\mathfrak{q}_{\varphi}(\chi) \mathfrak{o}^{\prime}=\ell_{\mathfrak{o}^{\prime}}\left(\left(\mathfrak{o}^{\prime} \otimes \operatorname{coker}(\underline{\varphi})\right)^{\chi}\right) / \ell_{\mathfrak{o}^{\prime}}\left(\left(\mathfrak{o}^{\prime} \otimes c l\right)^{\chi}\right)$.

The $\mathfrak{o}^{\prime}$-ideal $\mathfrak{q}_{\varphi}(\chi) \mathfrak{o}^{\prime}$ is the $q$-index localized by $\mathfrak{o} \rightarrow \mathfrak{o}^{\prime}$, so the computations to follow will take place in $K_{0} T\left(\mathfrak{o}^{\prime}\right)$ rather than in $K_{0} T(\mathfrak{o})$. This has the benefit that the localized Tate cohomology groups $H^{i}\left(G, \operatorname{Hom}_{\mathfrak{o}^{\prime}}\left(M, \mathfrak{o}^{\prime} \otimes_{\mathbb{Z}} X\right)\right)$ are all zero, where $X$ is a finitely generated $\mathbb{Z} G$-module.

We first look at the diagram

$$
\begin{aligned}
& c l \longmapsto \quad \nabla \quad \rightarrow \quad \bar{\nabla} \quad \text { which yields } \\
& \downarrow \varphi \quad \downarrow \bar{\varphi} \quad\left[\bar{\varphi}_{M}\right]-\left[\varphi_{M}\right]=\left[\operatorname{Hom}_{\mathfrak{o}^{\prime}}\left(M, \mathfrak{o}^{\prime} \otimes c l\right)_{G}\right] \\
& \begin{array}{ll}
\tilde{E}=\tilde{E} & =\left[\operatorname{Hom}_{\mathfrak{o}^{\prime}}\left(M, \mathfrak{o}^{\prime} \otimes c l\right)^{G}\right] \\
& =\left[\operatorname{Hom}_{\mathfrak{o}^{\prime} G}\left(\mathfrak{o}^{\prime} e_{\chi}, \mathfrak{o}^{\prime} \otimes c l\right)\right]=\left[\left(\mathfrak{o}^{\prime} \otimes c l\right)^{\chi}\right] \text { and }
\end{array}
\end{aligned}
$$

a)

$$
\left[\varphi_{M}\right]-\left[\bar{\varphi}_{M}\right]=-\left[\left(\mathfrak{o}^{\prime} \otimes c l\right)^{\chi}\right] .
$$

By restricting $\varphi_{+}$to ${ }^{0} W \rightarrow \mathbb{Z} G^{r}$ we arrive at the commuting diagram

$$
\begin{aligned}
& \Delta S \quad \bar{\nabla} \quad \rightarrow \quad{ }^{0} W
\end{aligned}
$$

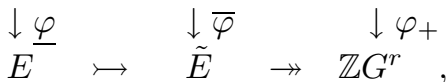

which, in $K_{0} T\left(\mathfrak{o}^{\prime}\right)$, gives

$$
\left[\bar{\varphi}_{M}\right]-\left[\underline{\varphi}_{M}\right]=\left[\left(\varphi_{+}\right)_{M}\right]
$$

From the definition of $\underline{\varphi}_{M}: \operatorname{Hom}_{\mathfrak{o}^{\prime}}\left(\mathfrak{o}^{\prime} e_{\chi}, \Delta S\right)_{G} \stackrel{\hat{G}}{\rightarrow} \operatorname{Hom}_{\mathfrak{o}^{\prime}}\left(\mathfrak{o}^{\prime} e_{\chi}, \Delta S\right)^{G} \stackrel{\underline{\varphi}}{\rightarrow}$ $\operatorname{Hom}_{\mathfrak{o}^{\prime}}\left(\mathfrak{o}^{\prime} e_{\chi}, E\right)^{G}$ we obtain

$$
\left[\underline{\varphi}_{M}\right]=\left[\left(\mathfrak{o}^{\prime} \otimes \operatorname{coker}(\underline{\varphi})\right)^{\chi}\right],
$$

since we are in $K_{0} T\left(\mathfrak{o}^{\prime}\right)$ and since taking $\chi$-eigenspaces is thus an exact functor.

Adding a), b), c) and applying $\ell_{\mathfrak{o}^{\prime}}$ will give the claim once we have shown that $\left[\left(\varphi_{+}\right)_{M}\right]=0$ in $K_{0} T\left(\mathfrak{o}^{\prime}\right)$. To that end write $\varphi_{+}=\bigoplus_{\mathfrak{p} \in S_{*}^{\text {ram }}} \operatorname{ind}_{G_{\mathfrak{p}}}^{G}\left(\varphi_{\mathfrak{p}} \sigma_{\mathfrak{p}}\right)$ and use Frobenius reciprocity in order to arrive at

$$
\left[\left(\varphi_{+}\right)_{M}\right]=\left[\left(\mathfrak{o}^{\prime} \otimes \operatorname{coker}\left(\varphi_{+}\right)\right)^{\chi}\right]=\sum_{\mathfrak{p} \in S_{*}^{\mathrm{ram}}}\left[\left(\mathfrak{o}^{\prime} \otimes \operatorname{coker}\left(\varphi_{\mathfrak{p}} \sigma_{\mathfrak{p}}\right)\right)^{\chi_{\mathfrak{p}}}\right]
$$

where $\chi_{\mathfrak{p}}$ is the restriction of $\chi$ to $G_{\mathfrak{p}}$. We are left with proving $\left[\left(\mathfrak{o}^{\prime} \otimes \operatorname{coker}\left(\varphi_{\mathfrak{p}} \sigma_{\mathfrak{p}}\right)\right)^{\chi_{\mathfrak{p}}}\right]$ $=0$ in $K_{0} T\left(\mathfrak{o}^{\prime}\right)$. 
For this we suppress the index $\mathfrak{p}$. If $\chi$ is non-trivial, then $e_{\chi}\left(\mathfrak{o}^{\prime} \otimes{ }^{0} W\right)=e_{\chi} \mathfrak{o}^{\prime} \kappa$ and $\varphi \sigma$ sends the element $e_{\chi} \kappa$ to

$$
e_{\chi} \varphi \sigma(\kappa)=e_{\chi} \varphi(|G| \kappa, 0)=e_{\chi}|G|(|G|-\hat{G})=|G|^{2} e_{\chi} .
$$

If $\chi$ is trivial, then $e_{\chi}\left(\mathfrak{o}^{\prime} \otimes{ }^{0} W\right)=e_{\chi} \mathfrak{o}^{\prime} \eta$ and $e_{\chi} \eta$ is sent to

$$
\begin{array}{r}
e_{\chi} \varphi((|G|-\hat{G}) \eta, 1)=e_{\chi} \varphi\left(-e \sum_{g \neq 1} a(g) g \kappa, 1\right) \\
=e_{\chi}(|G|-\hat{G}) \sum_{g \neq 1}-e a(g) g+e_{\chi} \hat{G}=|G| e_{\chi},
\end{array}
$$

where we have used Proposition 1(b). Since $\left|G_{\mathfrak{p}}\right|$ is a unit in $\mathfrak{o}^{\prime}$, we see that indeed $\left[\left(\mathfrak{o}^{\prime} \otimes \operatorname{coker}(\varphi \sigma)\right)^{\chi_{\mathfrak{p}}}\right]=0$.

Turning to $A_{\varphi}$, we define $\lambda_{+}: E \oplus \mathbb{Z} \oplus \mathbb{Z} G^{r} \rightarrow \mathbb{C} \otimes\left(\mathbb{Z} S \oplus{ }^{0} W\right)$ by $\lambda_{+}(a, z, b)=$ $\tilde{\lambda}(a, b)+\frac{z}{|G|}(\hat{G} \infty, 0)$ in order to get the following commutative diagram:

$$
\begin{aligned}
& \mathbb{C} \otimes \bar{\nabla} \longmapsto \mathbb{C} \otimes\left(\mathbb{Z} S \oplus^{0} W\right) \quad \rightarrow \quad \mathbb{C} \\
& \downarrow \bar{\varphi} \quad \downarrow \varphi_{+} \quad \| \\
& \mathbb{C} \otimes \tilde{E} \quad \longrightarrow \mathbb{C} \otimes\left(E \oplus \mathbb{Z} \oplus \mathbb{Z} G^{r}\right) \quad \rightarrow \quad \mathbb{C} \\
& \downarrow \tilde{\lambda} \quad \downarrow \lambda_{+} \quad \| \\
& \mathbb{C} \otimes \bar{\nabla} \quad \longmapsto \quad \mathbb{C} \otimes\left(\mathbb{Z} S \oplus{ }^{0} W\right) \quad \rightarrow \quad \mathbb{C}
\end{aligned}
$$

Composing the vertical arrows and applying $\operatorname{Hom}_{\mathbb{C} G}(V,-)$ we get

d) $\operatorname{det}\left(\tilde{\lambda} \varphi \mid \operatorname{Hom}_{\mathbb{C} G}(V, \mathbb{C} \otimes \nabla)\right)=\operatorname{det}\left(\lambda_{+} \varphi_{+} \mid \operatorname{Hom}_{\mathbb{C} G}\left(V, \mathbb{C} \otimes\left(\mathbb{Z} S \oplus^{0} W\right)\right)\right)$.

To handle this we build the commutative diagram

$$
\begin{array}{ccccc}
\mathbb{C} \otimes \mathbb{Z} S & \longmapsto & \mathbb{C} \otimes\left(\mathbb{Z} S \oplus^{0} W\right) & \rightarrow & \mathbb{C} \otimes{ }^{0} W \\
\downarrow \gamma_{1} & & \downarrow \lambda_{+} \varphi_{+} & & \downarrow \gamma_{2} \\
\mathbb{C} \otimes \mathbb{Z} S & \longmapsto & \mathbb{C} \otimes\left(\mathbb{Z} S \oplus^{0} W\right) & \rightarrow & \mathbb{C} \otimes{ }^{0} W
\end{array}
$$

where $\gamma_{1}(\mathfrak{p})=\lambda \underline{\varphi}(|G| \mathfrak{p}-\hat{G} \infty)+\frac{1}{|G|} \hat{G} \infty$ for $\mathfrak{p} \in S_{*}$ while $\gamma_{2}\left(\rho_{\mathfrak{p}}\right)=-\log (N \mathfrak{p}) \otimes$ $\left(y_{\mathfrak{p}}\left(\left|G_{\mathfrak{p}}\right|-\hat{G}_{\mathfrak{p}}\right)+\hat{G}_{\mathfrak{p}}\right) \rho_{\mathfrak{p}}$ for $\mathfrak{p} \in S_{*}^{\text {ram }}$. This holds because

$$
\begin{aligned}
\lambda_{+} \varphi_{+}(\mathfrak{p}, 0) & =\lambda_{+}(\underline{\varphi}(|G| \mathfrak{p}-\hat{G} \infty), 1,0) \\
& =\tilde{\lambda}(\underline{\varphi}(|G| \mathfrak{p}-\hat{G} \infty), 0)+\frac{1}{|G|}(\hat{G} \infty, 0) \\
& =\left(\lambda \underline{\varphi}(|G| \mathfrak{p}-\hat{G} \infty)+\frac{1}{|G|} \hat{G} \infty, 0\right)
\end{aligned}
$$

while $\lambda_{+} \varphi_{+}\left(0, \rho_{\mathfrak{p}}\right)=\lambda_{+}\left(0,1, \varphi_{\mathfrak{p}} \sigma_{\mathfrak{p}}\left(\rho_{\mathfrak{p}}\right)\right)$ with

$$
\begin{aligned}
\varphi_{\mathfrak{p}} \sigma_{\mathfrak{p}}\left(\rho_{\mathfrak{p}}\right) & =\varphi_{\mathfrak{p}}\left(\left|G_{\mathfrak{p}}\right| \rho_{\mathfrak{p}}-\hat{G}_{\mathfrak{p}} \eta_{\mathfrak{p}}, 1\right)=\varphi_{\mathfrak{p}}\left(\left(\left|G_{\mathfrak{p}}\right|-\hat{G}_{\mathfrak{p}}\right) \rho_{\mathfrak{p}}, 1\right) \\
& =\varphi_{\mathfrak{p}}\left(y_{\mathfrak{p}} \kappa_{\mathfrak{p}}, 1\right)=y_{\mathfrak{p}}\left(\left|G_{\mathfrak{p}}\right|-\hat{G}_{\mathfrak{p}}\right)+\hat{G}_{\mathfrak{p}} .
\end{aligned}
$$


By Proposition 1(a),(b) this yields

$$
\begin{aligned}
\lambda_{+} \varphi_{+}\left(0, \rho_{\mathfrak{p}}\right) & =\lambda_{+}\left(0,1, y_{\mathfrak{p}}\left(\left|G_{\mathfrak{p}}\right|-\hat{G}_{\mathfrak{p}}\right)+\hat{G}_{\mathfrak{p}}\right) \\
& =\tilde{\lambda}\left(0, y_{\mathfrak{p}}\left(\left|G_{\mathfrak{p}}\right|-\hat{G}_{\mathfrak{p}}\right)+\hat{G}_{\mathfrak{p}}\right)+\left(\frac{1}{|G|} \hat{G} \infty, 0\right)
\end{aligned}
$$

whose image in $\mathbb{C} \otimes{ }^{0} W$ is

$$
\tilde{\rho}\left(y_{\mathfrak{p}}\left(\left|G_{\mathfrak{p}}\right|-\hat{G}_{\mathfrak{p}}\right)+\hat{G}_{\mathfrak{p}}\right)=-\log (N \mathfrak{p}) \otimes\left(y_{\mathfrak{p}}\left(\left|G_{\mathfrak{p}}\right|-\hat{G}_{\mathfrak{p}}\right)+\hat{G}_{\mathfrak{p}}\right) \rho_{\mathfrak{p}}
$$

Now we get

$$
\begin{aligned}
& \operatorname{det}\left(\lambda_{+} \varphi_{+} \mid \operatorname{Hom}_{\mathbb{C} G}\left(V, \mathbb{C} \otimes\left(\mathbb{Z} S \oplus^{0} W\right)\right)\right) \\
& \text { e) } \quad=\operatorname{det}\left(\gamma_{1} \mid \operatorname{Hom}_{\mathbb{C} G}(V, \mathbb{C} \otimes \mathbb{Z} S)\right) \cdot \operatorname{det}\left(\gamma_{2} \mid \operatorname{Hom}_{\mathbb{C} G}\left(V, \mathbb{C} \otimes{ }^{0} W\right)\right)
\end{aligned}
$$

and we use the obvious diagram

$$
\begin{array}{ccccc}
\mathbb{C} \otimes \Delta S & \longmapsto & \mathbb{C} \otimes \mathbb{Z} S & \rightarrow & \mathbb{C} \\
\downarrow|G| \lambda \varphi & & \downarrow \gamma_{1} & & \| \\
\mathbb{C} \otimes \Delta \bar{S} & \longmapsto & \mathbb{C} \otimes \mathbb{Z} S & \rightarrow & \mathbb{C}
\end{array}
$$

to get

f) $\operatorname{det}\left(\gamma_{1} \mid \operatorname{Hom}_{\mathbb{C} G}(V, \mathbb{C} \otimes \mathbb{Z} S)\right)=|G|^{(\chi, \Delta S)} \operatorname{det}\left(\lambda \underline{\varphi} \mid \operatorname{Hom}_{\mathbb{C} G}(V, \mathbb{C} \otimes \Delta S)\right)$.

We next compute the determinant of $\gamma_{2}$ using the identification

$$
\operatorname{Hom}_{\mathbb{C} G}\left(V, \mathbb{C} \otimes{ }^{0} W\right)=e_{\chi}\left(\mathbb{C} \otimes{ }^{0} W\right),
$$

which originates in $V=\mathbb{C} G e_{\chi}$, and the $\mathbb{C}$-basis $\left\{e_{\chi} \rho_{\mathfrak{p}}: \mathfrak{p} \in S_{*}^{\mathrm{ram}}\right\}$ of $e_{\chi}\left(\mathbb{C} \otimes{ }^{0} W\right)$, which comes from the $\mathbb{C} G$-basis $\left\{\rho_{\mathfrak{p}}\right\}$ of $\mathbb{C} \otimes{ }^{0} W$, to compute

$$
\gamma_{2}\left(e_{\chi} \rho_{\mathfrak{p}}\right)=-\log (N \mathfrak{p}) \cdot \chi\left(y_{\mathfrak{p}}\left(\left|G_{\mathfrak{p}}\right|-\hat{G}_{\mathfrak{p}}\right)+\hat{G}_{\mathfrak{p}}\right) e_{\chi} \rho_{\mathfrak{p}}
$$

and therefore

g)

$$
\begin{aligned}
\operatorname{det}\left(\gamma_{2} \mid \operatorname{Hom}_{\mathbb{C} G}\left(V, \mathbb{C} \otimes{ }^{0} W\right)\right) & \\
= & \prod_{\mathfrak{p} \in S_{*}^{\text {ram }}}\left[-\log (N \mathfrak{p}) \cdot \chi\left(y_{\mathfrak{p}}\left(\left|G_{\mathfrak{p}}\right|-\hat{G}_{\mathfrak{p}}\right)+\hat{G}_{\mathfrak{p}}\right)\right] .
\end{aligned}
$$

Combining the formulas d), e), f), g) with the definition of $A_{\varphi}$ we then get

$$
\begin{aligned}
A_{\varphi}(\check{\chi})= & \frac{\operatorname{det}\left(\lambda \underline{\varphi} \mid \operatorname{Hom}_{\mathbb{C} G}(V, \mathbb{C} \otimes \Delta S)\right)}{c_{S}(\check{\chi})} \\
& \cdot|G|^{(\chi, \Delta S)} \prod_{\mathfrak{p} \in S_{*}^{\text {ram }}}\left[-\frac{1}{e_{\mathfrak{p}}} \chi\left(y_{\mathfrak{p}}\left(\left|G_{\mathfrak{p}}\right|-\hat{G}_{\mathfrak{p}}\right)+\hat{G}_{\mathfrak{p}}\right)\right]
\end{aligned}
$$

which together with the claim reduces to showing that $\chi\left(y_{\mathfrak{p}}\left(\left|G_{\mathfrak{p}}\right|-\hat{G}_{\mathfrak{p}}\right)+\hat{G}_{\mathfrak{p}}\right)$ is a unit in $\mathfrak{o}^{\prime}$. 
If $\left.\chi\right|_{G_{\mathfrak{p}}}=1$ this is $\left|G_{\mathfrak{p}}\right|$; if $\left.\chi\right|_{G_{\mathfrak{p}}} \neq 1$ it is $\left|G_{\mathfrak{p}}\right| \chi\left(y_{\mathfrak{p}}\right)$ with $y_{\mathfrak{p}}$ as in Proposition 1 . But if $\left.\chi\right|_{I_{\mathfrak{p}}} \neq 1$ we get $\chi\left(y_{\mathfrak{p}}\right)=-\left|G_{\mathfrak{p}}\right| e_{\mathfrak{p}}$, while if $\left.\chi\right|_{I_{\mathfrak{p}}}=1$ then $\left(\sum_{i=1}^{f_{\mathfrak{p}}-1} i \phi_{\mathfrak{p}}^{-i}\right) \times$ $\left(1-\phi_{\mathfrak{p}}^{-1}\right)=\left\langle\hat{\phi}_{\mathfrak{p}}\right\rangle-f_{\mathfrak{p}}$ implies $\chi\left(y_{\mathfrak{p}}\right)=e_{\mathfrak{p}}^{2} \chi\left(\sum_{i=1}^{f_{\mathfrak{p}}-1} i \phi_{\mathfrak{p}}^{-i}\right)=\frac{-e_{\mathfrak{p}}^{2} f_{\mathfrak{p}}}{1-\chi_{\mathfrak{p}}\left(\phi_{\mathfrak{p}}^{-1}\right)}$ is again of the type required.

\section{9. l-ADIC REDUCTIONS}

In this section, which, for the convenience of the reader, repeats the contents of [We, $\S 14]$, we assume the validity of Stark's conjecture, so, by Lemma $3, \mathfrak{a}(\chi)$ is a fractional $\mathfrak{o}$-ideal in $F$ satisfying $\mathfrak{a}(\chi)^{\gamma}=\mathfrak{a}\left(\chi^{\gamma}\right)$ for $\gamma \in \Gamma$.

For a prime $l$ denote by $\mathbb{C}_{l}$ a fixed completion of an algebraic closure of the $l$ adic rationals $\mathbb{Q}_{l}$. Let $F_{l}$ be a subfield of $\mathbb{C}_{l}$ so that $F_{l} / \mathbb{Q}_{l}$ is finite Galois containing a primitive $|G|^{\text {th }}$ root of unity. Suppose $i_{l}: F \rightarrow F_{l}$ is a field embedding. If $\chi_{l}$ is a $\mathbb{C}_{l}$-character of $G$, then $\chi_{l}=i_{l} \chi$ for some $F$-character $\chi$ and we define $\mathfrak{a}^{(l)}\left(\chi_{l}\right)=i_{l}(\mathfrak{a}(\chi)) \mathfrak{o}_{l}$ where $\mathfrak{o}_{l}$ is the ring of integers of $F_{l}$.

Proposition 11. (a) $\mathfrak{a}^{(l)}$ is independent of the choice of $i_{l}$.

(b) Suppose $\mathfrak{a}_{K^{\prime} / k^{\prime}}^{(l)}\left(\chi_{l}\right)=(1)$ whenever $\chi_{l}$ is a non-trivial linear $\mathbb{C}_{l}$-character of a cyclic subextension $K^{\prime} / k^{\prime}$ of $K / k$ which has degree prime to l. Then $\mathfrak{a}_{K / k}(\chi)$ is relatively prime to $l$ for all characters $\chi$ of $G$.

(c) Let $K / \mathbb{Q}$ be abelian. Then the above statement in (b) remains true with $K^{\prime} / k^{\prime}$ replaced by cyclic subextensions of $K / \mathbb{Q}$ of degree prime to l such that $k^{\prime} / \mathbb{Q}$ are cyclic l-extensions.

Proof. (a) If $i_{l}^{\prime}$ is another embedding, then $i_{l}^{\prime}=i_{l} \gamma$ for some $\gamma \in \Gamma$, so $\chi_{l}=i_{l}^{\prime} \chi^{\prime}$ implies $\chi_{l}=i_{l} \chi$ with $\chi=\left(\chi^{\prime}\right)^{\gamma}$, hence $i_{l}(\mathfrak{a}(\chi))=i_{l}\left(\mathfrak{a}\left(\chi^{\prime}\right)^{\gamma}\right)=i_{l}^{\prime}\left(\mathfrak{a}\left(\chi^{\prime}\right)\right)$.

(b) The embedding $i_{l}$ induces a prime $\mathfrak{l}$ on $F$ above $l$. Let $I$ denote the inertia subgroup of $\mathfrak{l}$ in $\Gamma$ with fixed field $F^{\prime}$. Let $\chi^{\prime}=m \sum_{\gamma \in I} \chi^{\gamma}$, an $F^{\prime}$-rational character $\left(m \in \mathbb{Z}\right.$ appropriate). Then $\mathfrak{a}\left(\chi^{\prime}\right)=\left[\prod_{\gamma \in I} \mathfrak{a}(\chi)^{\gamma}\right]^{m}$. As $I$ fixes $\mathfrak{l}, \mathfrak{a}\left(\chi^{\prime}\right)$ is prime to $\mathfrak{l}$ if, and only if, $\mathfrak{l}$ is prime to $\mathfrak{a}(\chi)$.

Now $i_{l} \chi^{\prime}$ is defined over $F_{l}^{\prime}$ which is the maximal unramified subfield in $F_{l} / \mathbb{Q}_{l}$. By the claim below some power of $i_{l}\left(\mathfrak{a}\left(\chi^{\prime}\right)\right) \mathfrak{o}_{l}=\mathfrak{a}^{(l)}\left(i_{l}\left(\chi^{\prime}\right)\right)$ is the product of values of $\mathfrak{a}^{(l)}$ on characters induced from those in the hypothesis, from which (b) follows by the $l$-analogue of Proposition 9.

Claim. Assume that $F_{l}^{\prime} / \mathbb{Q}_{l}$ is an unramified extension containing all $|G|^{\text {th }}$ roots of unity of order prime to $l$. Then every $F_{l}^{\prime}$-character of $G$ is a $\mathbb{Q}$-linear combination of characters induced from linear characters of cyclic subquotients of $G$ of order prime to $l$.

Proof of the Claim. By Artin's induction theorem we may assume that $G$ is cyclic. Decompose $G=G_{l} \times G^{\prime}$ into $l$ - and $l^{\prime}$-parts. Since the $l$-power cyclotomic polynomials are irreducible over $F_{l}^{\prime}$, there is a unique faithful irreducible $F_{l}^{\prime}$-character of $G_{l}$ and this is a $\mathbb{Z}$-linear combination of permutation characters. On the other hand, $F_{l}^{\prime} G^{\prime}$ splits completely.

The proof of (c) is similar. Induce the given $\chi$ up to $G(K / \mathbb{Q})$, then decompose and inflate. Once more by the $l$-adic analogue of Proposition 9 it is enough to look at the situation when $k=\mathbb{Q}$ and $K / \mathbb{Q}$ is cyclic. Applying (b) then gives the assertion. 


\section{The RAmachandRa isogeny}

From now on $K / k$ is an extension of number fields which is contained in a cyclotomic field and $S$ is the set of all infinite primes of $K$.

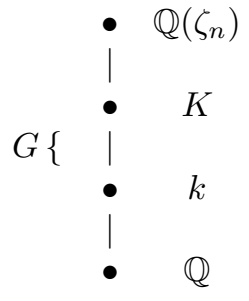

Choose and fix a positive integer $n \neq 2 \bmod 4$ so that $K$ can be identified with a subfield of $\mathbb{Q}(\zeta)$, where $\zeta=\zeta_{n}$ is a primitive $n^{\text {th }}$ root of unity. Then $\zeta \mapsto e^{2 \pi i / n}$ embeds $\mathbb{Q}(\zeta)$ into $\mathbb{C}$ and the restriction to $K$ shall be our distinguished infinite prime $\infty \in S_{*}$.

As in [Wa, p.147] we write $n=\prod_{i=1}^{s} p_{i}^{e_{i}}$ and $n_{I}=\prod_{i \in I} p_{i}^{e_{i}}$ for each proper subset $I$ of $\{1, \ldots, s\}$.

Definition. (i) $\xi_{n}=\prod_{I}\left(1-\zeta^{n_{I}}\right)\left(1-\zeta^{-n_{I}}\right)$.

(ii) $\xi_{K}=N_{\mathbb{Q}(\zeta) / K}\left(\xi_{n}\right)$, where $N_{\mathbb{Q}(\zeta) / K}$ denotes the norm from $\mathbb{Q}(\zeta)$ to $K$.

Observe that $\xi^{\sigma-1}$ is a real unit in $\mathbb{Q}(\zeta)$ for any $\sigma$ in the Galois group $G(\mathbb{Q}(\zeta) / \mathbb{Q})$ of $\mathbb{Q}(\zeta)$ over $\mathbb{Q}$. Since the $G$-stabilizer of $\infty$ fixes $\xi_{K}$, there is a unique $G$-homomorphism $\mathbb{Z} S \rightarrow K^{\times}$sending $\infty$ to $\xi_{K}$. It restricts to a map $\Delta S \rightarrow E$ which we define to be $\underline{\varphi}$.

Proposition 12. Let $\chi$ be a non-trivial irreducible character of $G$ with representation space $V$. Then

$$
\frac{\operatorname{det}\left(\lambda \underline{\varphi} \mid \operatorname{Hom}_{\mathbb{C G}}(V, \mathbb{C} \otimes \Delta S)\right)}{c_{S}(\check{\chi})}=\prod_{\substack{\psi \mid \chi \\ \text { even }}}\left[-4 \prod_{p_{i} \nmid f_{\psi}}\left(\Phi\left(p_{i}^{e_{i}}\right)+1-\check{\psi}\left(p_{i}\right)\right)\right] \prod_{\substack{\psi \mid \chi \\ \text { odd }}}\left(-B_{1, \check{\psi}}\right)^{-1}
$$

where $\psi$ runs through all characters of $G(K / \mathbb{Q})$ extending $\chi$ and $f_{\psi}$ is the conductor of $\psi$. The symbol $\breve{\psi}\left(p_{i}\right)$ is the value of $\check{\psi}$ on the Frobenius automorphism for $p_{i}$, i.e. $\breve{\psi}$ is interpreted as a Dirichlet character modulo $n / p_{i}^{e_{i}}$. We say that $\psi$ is even if $\operatorname{infl}_{G(K / \mathbb{Q})}^{G(\mathbb{Q}(\zeta)} \psi$ is trivial on complex conjugation; otherwise it is odd, and $B_{1, \tilde{\psi}}$ is the generalized Bernoulli number [Wa, p.30]. Finally, $\Phi$ is the Euler phi function.

Proof. As before, let $e_{\chi}=\frac{1}{|G|} \sum_{g \in G} \chi\left(g^{-1}\right) g$ be the primitive idempotent for $\chi$. Thus $e_{\chi}=\sum_{\psi \mid \chi} e_{\psi}, V=\mathbb{C} G e_{\chi}$ and so, as $\chi \neq 1$,

$$
\operatorname{Hom}_{\mathbb{C} G}(V, \mathbb{C} \otimes \Delta S)=e_{\chi}(\mathbb{C} \otimes \Delta S)=e_{\chi} \mathbb{C} S=\sum_{\psi \mid \chi} e_{\psi} \mathbb{C} S=\sum_{\substack{\psi \mid \chi \\ \text { even }}} e_{\psi} \mathbb{C} S .
$$

It follows that $\left\{e_{\psi} \infty: \psi \mid \chi, \psi\right.$ even $\}$ is a basis of $\operatorname{Hom}_{\mathbb{C} G}(V, \mathbb{C} \otimes \Delta S)$ under the above identification. On writing $e_{\psi}=\frac{1}{[K: \mathbb{Q}]} \sum_{\sigma \in G(K / \mathbb{Q})} \psi\left(\sigma^{-1}\right)(\sigma-1)$ and $K^{+}$for 
the maximal real subfield of $K$ we compute

$$
\begin{aligned}
\lambda \underline{\varphi}\left(e_{\psi} \infty\right) & \frac{1}{[K: \mathbb{Q}]} \sum_{\sigma} \psi\left(\sigma^{-1}\right) \lambda\left(\xi_{K}^{\sigma-1}\right) \\
= & \frac{1}{[K: \mathbb{Q}]} \sum_{\sigma} \psi\left(\sigma^{-1}\right) \frac{1}{\left[K: K^{+}\right]} \sum_{\sigma^{\prime}} \log \left|\xi_{K}^{\sigma-1}\right|_{\sigma^{\prime} \infty} \sigma^{\prime} \infty \\
= & \frac{1}{\left[K: K^{+}\right][K: \mathbb{Q}]} \sum_{\sigma, \sigma^{\prime}} \psi\left(\sigma^{-1}\right) \log \left|\xi_{K}^{\left(\sigma^{\prime}\right)^{-1}(\sigma-1)}\right|_{\infty} \sigma^{\prime} \infty \\
= & \frac{1}{\left[K: K^{+}\right][K: \mathbb{Q}]} \sum_{\sigma, \sigma^{\prime}} \psi\left(\left(\sigma \sigma^{\prime}\right)^{-1}\right) \log \left|\xi_{K}^{\left(\sigma^{\prime}\right)^{-1}\left(\sigma \sigma^{\prime}-1\right)}\right|_{\infty} \sigma^{\prime} \infty \\
= & \frac{1}{\left[K: K^{+}\right][K: \mathbb{Q}]} \sum_{\sigma} \sum_{\sigma^{\prime}} \psi\left(\sigma^{-1}\right) \log \left|\xi_{K}^{\sigma}\right|_{\infty} \psi\left(\left(\sigma^{\prime}\right)^{-1}\right) \sigma^{\prime} \infty \\
& -\frac{1}{\left[K: K^{+}\right][K: \mathbb{Q}]} \sum_{\sigma^{\prime}} \sum_{\sigma} \psi\left(\sigma^{-1}\right) \psi\left(\left(\sigma^{\prime}\right)^{-1}\right) \log \left|\xi_{K}^{\left(\sigma^{\prime}\right)^{-1}}\right|_{\infty} \sigma^{\prime} \infty \\
= & \frac{1}{\left[K: K^{+}\right]}\left(\sum_{\sigma} \psi\left(\sigma^{-1}\right) \log \left|\xi_{K}^{\sigma}\right|_{\infty}\right) e_{\psi} \infty-0 .
\end{aligned}
$$

Hence, with respect to the chosen basis, $\lambda \underline{\varphi}$ has diagonal matrix with diagonal entries

$$
\begin{gathered}
\frac{1}{\left[K: K^{+}\right]} \sum_{\sigma} \psi\left(\sigma^{-1}\right) \log \left|\xi_{K}^{\sigma}\right|_{\infty}=\sum_{\sigma \in G(\mathbb{Q}(\zeta) / \mathbb{Q})} \psi\left(\sigma^{-1}\right) \log \left|\xi_{n}^{\sigma}\right| \\
=2 \sum_{\sigma \in G(\mathbb{Q}(\zeta) / \mathbb{Q})} \check{\psi}(\sigma) \sum_{I} \log \left|\left(1-\zeta^{n_{I}}\right)^{\sigma}\right|,
\end{gathered}
$$

since $|\cdot|_{\infty}=|\cdot|^{\left[K: K^{+}\right]}$and $\xi_{n}=\prod_{I}\left(-\zeta^{-n_{I}}\right)\left(1-\zeta^{n_{I}}\right)^{2}$. Identifying $G(\mathbb{Q}(\zeta) / \mathbb{Q})$ and $(\mathbb{Z} / n)^{\times}$we arrive at

$$
2 \sum_{a \in(\mathbb{Z} / n)^{\times}} \check{\psi}(a) \sum_{I} \log \left|1-\zeta^{a n_{I}}\right|=-2 \tau_{w}(\check{\psi}) L(1, \psi) \prod_{p_{i} \nmid f_{\psi}}\left(\Phi\left(p_{i}^{e_{i}}\right)+1-\check{\psi}\left(p_{i}\right)\right)
$$

by combining the formulae ${ }^{3}$ on lines -1 and -7 in [Wa, p.149]. Because of the functional equation for the $L$-series, which in our situation reads [Wa, p.35]

$$
\left(\frac{f_{\psi}}{\pi}\right)^{s / 2} \Gamma(s / 2) L(s, \check{\psi})=W_{\breve{\psi}}\left(\frac{f_{\psi}}{\pi}\right)^{(1-s) / 2} \Gamma\left(\frac{1-s}{2}\right) L(1-s, \psi),
$$

where $W_{\tilde{\psi}}$ is the Artin root number of $\check{\psi}$, we obtain, since $\Gamma(1 / 2)=\pi^{1 / 2}$ and $\Gamma$ has a simple pole with residue 1 at $s=0: 2 L^{\prime}(0, \check{\psi})=W_{\check{\psi}} f_{\psi}^{1 / 2} L(1, \psi)=\tau_{w}(\check{\psi}) L(1, \psi)$.

Collecting terms we have

$$
\operatorname{det}\left(\lambda \underline{\varphi} \mid \operatorname{Hom}_{\mathbb{C} G}(V, \mathbb{C} \otimes \Delta S)\right)=\prod_{\substack{\psi \\ \psi<\\ \text { even }}}\left[-4 L^{\prime}(0, \check{\psi}) \prod_{p_{i} \nmid f_{\psi}}\left(\Phi\left(p_{i}^{e_{i}}\right)+1-\check{\psi}\left(p_{i}\right)\right)\right] .
$$

\footnotetext{
${ }^{3}$ Later on we will have to look at Galois Gauss sums as appearing in [Fr1]. Washington's Gauss sum, which for clarity reasons we denote by $\tau_{w}(\breve{\psi})$, will then have to be replaced by an $l$-adic Gauss sum.
} 
So the proposition follows from the identity

$$
c_{S}(\check{\chi})=\prod_{\substack{\psi \mid \chi \\ \text { even }}} L^{\prime}(0, \check{\psi}) \prod_{\substack{\psi \mid \chi \\ \text { odd }}} L(0, \check{\psi})=\prod_{\substack{\psi \mid \chi \\ \text { even }}} L^{\prime}(0, \check{\psi}) \prod_{\substack{\psi \mid \chi \\ \text { odd }}}\left(-B_{1, \check{\psi}}\right),
$$

which holds because of [Wa, p.31] and

$$
\begin{gathered}
\operatorname{ind}_{G}^{G(K / \mathbb{Q})} \chi=\sum_{\psi \mid \chi} \psi, L(s, \check{\chi})=\prod_{\psi \mid \chi} L(s, \check{\psi}), \\
L(s, \check{\psi})=\left\{\begin{array}{cc}
L^{\prime}(0, \check{\psi}) s+\cdots & \text { if } \psi \text { is even, } \\
L(0, \check{\psi})+\cdots & \text { if } \psi \text { is odd. }
\end{array}\right.
\end{gathered}
$$

Remark. The proposition implies that $\underline{\varphi}$ is an isogeny and that Stark's conjecture is true in our situation.

\section{Plan of proof of Theorem A}

In order to show that $\mathfrak{a}(\chi)=(1)$ we show that $\mathfrak{a}(\chi)$ is relatively prime to $l$ for every prime $l$ and every character $\chi$.

Fix a prime $l$. By Proposition 11 it suffices to prove $\mathfrak{a}^{(l)}(\chi)=(1)$ for every non-trivial linear $\mathbb{C}_{l}$-character $\chi=\chi_{l}$ in the special case when $K / \mathbb{Q}$ is cyclic with $l \nmid[K: k]$ and $[k: \mathbb{Q}]$ an $l$-power. Moreover, by the $l$-adic analogue of Proposition 9 we may assume $\chi$ is faithful.

Let $\mathfrak{o}_{l}$ be the ring of integers of a field $F_{l} \subset \mathbb{C}_{l}$ as in $\S 9$ and large enough that there is an embedding $i_{l}: F \rightarrow F_{l}$. With $S$ and $\underline{\varphi}: \Delta S \rightarrow E$ as in $\S 10$ we can combine Propositions 11 and 12 to obtain

$$
\mathfrak{a}^{(l)}(\chi)=\frac{\prod_{\text {even }}^{\psi \mid \chi}\left(4 \prod_{p_{i} \nmid f_{\psi}}\left(\Phi\left(p_{i}^{e_{i}}\right)+1-\check{\psi}\left(p_{i}\right)\right)\right)}{\prod_{\psi \mid \chi, \text { odd }} B_{1, \check{\psi}}} \cdot \frac{\ell\left(\left(\mathfrak{o}_{l} \otimes c l\right)^{\chi}\right)}{\ell\left(\left(\mathfrak{o}_{l} \otimes \operatorname{coker} \underline{\varphi}\right)^{\chi}\right)}
$$

because $i_{l}$ takes $\mathfrak{o}^{\prime}=\mathfrak{o}[1 /|G|]$ into $\mathfrak{o}_{l}$, as $l \nmid|G|$. Here, $\ell=\ell_{\mathfrak{o}_{l}}$ and $\psi$ runs through the linear $\mathbb{C}_{l}$-characters of $G(K / \mathbb{Q})$ extending $\chi$.

Let us say that $\chi$ is even, respectively odd, if $k$ is real and $\chi$ is trivial, respectively non-trivial, on complex conjugation when restricted to $K$ (so considered in $G$ ). In particular, $k$ is now real until the last step.

Examining the lengths in the above formulae we will show

$$
\begin{aligned}
& \frac{\ell\left(\left(\mathfrak{o}_{l} \otimes c l\right)^{\chi}\right)}{\ell\left(\left(\mathfrak{o}_{l} \otimes \operatorname{coker} \varphi\right)^{\chi}\right)}= \\
& \begin{cases}\frac{\ell\left(\left(\mathfrak{o}_{l} \otimes c l\right)^{\chi}\right)}{\ell\left(\left(\left(_{l} \otimes \mu\right)^{\chi}\right)\right.} & \text { if } \chi \text { is odd, } \\
\frac{\left.\ell\left(\mathfrak{o}_{l} \otimes \mathbb{Z}_{l} G(M / K)\right)^{\chi}\right)}{L_{l}(1, \chi)} \prod_{\psi \mid \chi}\left(2 \prod_{p_{i} \nmid f_{\psi}}\left(\Phi\left(p_{i}^{e_{i}}\right)+1-\check{\psi}\left(p_{i}\right)\right)\right)^{-1} & \text { if } \chi \text { is even, }\end{cases}
\end{aligned}
$$

where $M$ denotes the maximal abelian $l$-extension of $K$ which is unramified outside $l, L_{l}(s, \chi)$ is the $l$-adic $L$-function and $\mu$ is the group of roots of unity in $K$.

This result for $\chi$ odd follows by tensoring the diagram below with $\mathfrak{o}_{l}$ and taking $\chi$-eigenspaces, because complex conjugation acts trivially on $E / \mu$, hence $\left(\mathfrak{o}_{l} \otimes E / \mu\right)^{\chi}=0:$

$$
\begin{array}{cccc}
\Delta S & = & \Delta S \\
& \downarrow & & \\
& \downarrow & & \downarrow \\
& & & \\
E & \rightarrow & E / \mu
\end{array}
$$


In the even case $K$ is real. Consider the natural embedding $E \rightarrow \prod_{\mathfrak{P} \mid l} U_{\mathfrak{P}}$, where $U_{\mathfrak{P}}$ denotes the units of the completion $K_{\mathfrak{P}}$ of $K$ at a prime $\mathfrak{P} \mid l$. It induces $\alpha: \mathbb{Z}_{l} \otimes E \rightarrow \prod_{\mathfrak{P} \mid l} U_{\mathfrak{P}}^{1}$ with $U_{\mathfrak{P}}^{1}$ the principal units in $K_{\mathfrak{P}}$, since $l \nmid\left|U_{\mathfrak{P}}: U_{\mathfrak{P}}^{1}\right|$. By a theorem of Brumer [Br] $\alpha$ is injective. From [Gr1, pp.153-154] or [Wa, p.268, take the $l$-part] we know that $\operatorname{coker}(\alpha)$ is canonically isomorphic to the Galois group $G(M / L)$ where $L$ is the maximal unramified l-extension of $K$, hence $G(L / K) \simeq$ $\mathbb{Z}_{l} \otimes c l$. Since $K$ is real, the cyclotomic $l$-extension $K_{\infty}$ of $K$ equals $K k_{\infty}$, with $k_{\infty}$ denoting the cyclotomic $l$-extension of $k$, and so $K_{\infty} / k$ is abelian. Moreover, $M$ is finite over $K_{\infty}$ by [Wa, pp.266-267]. Since $\chi \neq 1$, this implies the finiteness of $\left(\mathfrak{o}_{l} \otimes_{\mathbb{Z}_{l}} G(M / K)\right)^{\chi}$.

We compose $\alpha$ with $\varphi$ in order to get the injection

$$
\mathbb{Z}_{l} \otimes \Delta S \stackrel{\varphi}{\longmapsto} \mathbb{Z}_{l} \otimes E \stackrel{\alpha}{\longmapsto} \prod_{\mathfrak{P} \mid l} U_{\mathfrak{P}}^{1} .
$$

Tensoring with $\mathfrak{o}_{l}$ over $\mathbb{Z}_{l}$ we obtain

$$
\frac{\ell\left(\left(\mathfrak{o}_{l} \otimes c l\right)^{\chi}\right)}{\ell\left(\left(\mathfrak{o}_{l} \otimes \operatorname{coker}(\underline{\varphi})\right)^{\chi}\right)}=\frac{\ell\left(\left(\mathfrak{o}_{l} \otimes_{\mathbb{Z}_{l}} G(L / K)\right)^{\chi}\right) \cdot \ell\left(\left(\mathfrak{o}_{l} \otimes_{\mathbb{Z}_{l}} G(M / L)\right)^{\chi}\right)}{\ell\left(\left(\mathfrak{o}_{l} \otimes_{\mathbb{Z}_{l}} \operatorname{coker}(\alpha \underline{\varphi})\right)^{\chi}\right)}
$$

and apply Theorem 14 in $\S 12$ in order to arrive at (2).

Combining (1) and (2) yields

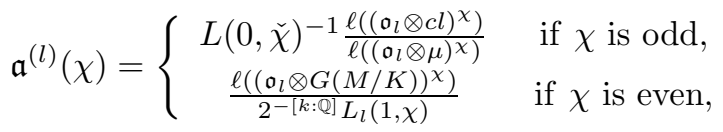

since, in the odd case, $\prod_{\psi \mid \chi}\left(-B_{1, \check{\psi}}\right)=\prod_{\psi \mid \chi} L(0, \check{\psi})=L(0, \check{\chi})$, with the last equality reflecting the induction property of the $L$-series.

From Iwasawa theory we finally get that $\mathfrak{a}^{(l)}(\chi)=(1)$ in both cases of the above formula, by Theorem 15 in the even case and by [Wi2, Theorem 3] in the odd case.

With respect to the latter observe that in Wiles' theorem the field $F_{\chi}$ is our $K$ and so the group $H_{\chi}$ there is the class group $c l$, up to $G$-isomorphism. Consequently, by the theorem,

$$
\left|\left(\mathfrak{o}_{l} \otimes c l\right)^{\chi}\right| \sim_{l} L(0, \check{\chi})^{\left[\mathfrak{o}_{l}: \mathbb{Z}_{l}\right]}\left|\left(\mathfrak{o}_{l} \otimes \mu\right)^{\chi}\right|,
$$

as its extra hypothesis is satisfied by [Wi2, p.556, line -4], since $l \neq 2$ if $\chi$ is odd. The odd case follows because of the relation $\left|\mathfrak{o}_{l} \otimes X\right| \mathfrak{o}_{l}=\ell\left(\mathfrak{o}_{l} \otimes X\right)^{\left[\mathfrak{o}_{l}: \mathbb{Z}_{l}\right]}$ between the group order and the $\mathfrak{o}_{l}$-length of a finite group $X$.

Finally we must reduce the general case to the case that $k$ is real. Let $k^{+}$be the maximal real subfield of $k$ and assume $k \neq k^{+}$. Since $k / \mathbb{Q}$ has $l$-power degree this forces $l=2$.

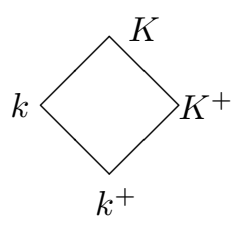

Decompose $\operatorname{ind}_{G}^{G\left(K / k^{+}\right)} \chi$ into irreducible characters and let $\chi^{+}$be the character of $G\left(K^{+} / k^{+}\right)$, with $K^{+}$the maximal real subfield of $K$, which inflates to one of the two constituents of $\operatorname{ind}_{G}^{G\left(K / k^{+}\right)} \chi$. Then the extensions of $\chi^{+}$to characters of $G\left(K^{+} / \mathbb{Q}\right)$ inflate to the even extensions $\psi$ of $\chi$. 
By (1) we thus have, using an obvious notation,

$$
\frac{\mathfrak{a}^{(2)}(\chi)}{\mathfrak{a}^{(2)}\left(\chi^{+}\right)}=\prod_{\substack{\psi \mid \chi \\ \text { odd }}} B_{1, \check{\psi}}^{-1} \cdot \frac{\ell\left(\left(\mathfrak{o}_{2} \otimes c l\right)^{\chi}\right) \cdot \ell\left(\left(\mathfrak{o}_{2} \otimes \operatorname{coker} \underline{\varphi}^{+}\right)^{\chi^{+}}\right)}{\ell\left(\left(\mathfrak{o}_{2} \otimes c l^{+}\right)^{\chi^{+}}\right) \cdot \ell\left(\left(\mathfrak{o}_{2} \otimes \operatorname{coker} \underline{\varphi}\right)^{\chi}\right)}
$$

At this stage we look at

$$
\begin{aligned}
& \Delta S \stackrel{\text { res }}{\longrightarrow} \Delta S^{+}
\end{aligned}
$$

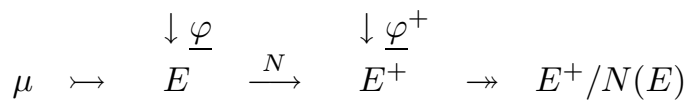

The action of $G$ on $\Delta S^{+}$and $E^{+}$can, via the natural isomorphism $G \simeq G^{+}=$ $G\left(K^{+} / k^{+}\right)$, be identified with that of $G^{+}$. Taking now $\chi$-eigenspaces we obtain, in $K_{0} T\left(\mathfrak{o}_{2}\right)$,

$\left[\left(\mathfrak{o}_{2} \otimes \operatorname{coker} \underline{\varphi}^{+}\right)^{\chi^{+}}\right]-\left[\left(\mathfrak{o}_{2} \otimes \operatorname{coker} \underline{\varphi}\right)^{\chi}\right]=\left[\left(\mathfrak{o}_{2} \otimes E^{+} / N(E)\right)^{\chi^{+}}\right]-\left[\left(\mathfrak{o}_{2} \otimes \mu\right)^{\chi}\right]$.

The exact sequence $E / \mu E^{+} \stackrel{N}{\longmapsto} E^{+} /\left(E^{+}\right)^{2} \rightarrow E^{+} / N(E)$, with left term $E / \mu E^{+}$ a cyclic group of order 1 or 2 [Wa, Theorem 4.12, p.39], hence $G$-trivial, yields $\left.\left[\left(\mathfrak{o}_{2} \otimes E^{+} / N(E)\right)^{\chi^{+}}\right]=\left[\left(\mathfrak{o}_{2} \otimes E^{+} /\left(E^{+}\right)\right)^{2}\right)^{\chi^{+}}\right]$, as $\chi \neq 1$.

Since $\mathbb{Q}_{2} \otimes E^{+} \simeq \mathbb{Q}_{2} \otimes \Delta S^{+}$and since $G^{+}$has odd order, we have $\mathfrak{o}_{2} \otimes\left(E^{+} / \pm 1\right) \simeq$ $\mathfrak{o}_{2} \otimes \Delta S^{+}$, hence $\ell\left(\left(\mathfrak{o}_{2} \otimes E^{+} /\left(E^{+}\right)^{2}\right)^{\chi^{+}}\right)=\ell\left(\left(\mathfrak{o}_{2} \otimes \mathbb{Z} S^{+} / 2 \mathbb{Z} S^{+}\right)^{\chi^{+}}\right)=2^{\left[k^{+}: \mathbb{Q}\right]} \mathfrak{o}_{2}$, because $\chi^{+} \neq 1$ and $\mathbb{Z} S^{+}$has $G^{+}$-character $\left[k^{+}: \mathbb{Q}\right] \operatorname{ind}_{1}^{G^{+}}(1)$.

As $\mathfrak{a}^{(2)}\left(\chi^{+}\right)=1$ by $k^{+}$real, we have

$$
\mathfrak{a}^{(2)}(\chi)=\frac{\ell\left(\left(\mathfrak{o}_{2} \otimes c l^{-}\right)^{\chi}\right)}{\ell\left(\left(\mathfrak{o}_{2} \otimes \mu\right)^{\chi}\right)} \cdot\left(\prod_{\operatorname{odd} \psi \mid \chi} \frac{1}{2} B_{1, \check{\psi}}\right)^{-1}
$$

where $\mathrm{cl}^{-}$is defined by the exact sequence $c l^{-} \longmapsto c l \stackrel{N}{\rightarrow} c l^{+}$[Wa, Theorem 10.1, p.184].

Assume now that 2 does not ramify in $K$. Then $\mu=\{ \pm 1\}$, so $\ell\left(\left(\mathfrak{o}_{2} \otimes \mu\right)^{\chi}\right)=(1)$. Therefore $\mathfrak{a}^{(2)}(\chi)=(1)$ is equivalent to

$$
\ell\left(\left(\mathfrak{o}_{2} \otimes c l^{-}\right)^{\chi}\right)=\prod_{\text {odd } \psi \mid \chi} \frac{1}{2} B_{1, \check{\psi}},
$$

which follows from [Gc, Theorem A, p.453] on writing

$$
\ell\left(\left(\mathfrak{o}_{2} \otimes c l^{-}\right)^{\chi}\right)=\prod_{\operatorname{odd} \psi \mid \chi} \ell\left(\left(\mathfrak{o}_{2} \otimes c l^{-}\right)_{\psi}\right) .
$$

12. l-ADIC LOGARITHMS AND L-FUnCTIONS, RESOLVENTS AND GAUSS SUMS

We shall need a small variation of an old trick [Wa, p.71].

Lemma 13. Let $G$ be a subgroup of an abelian group $H$ and $f: H \rightarrow \mathbb{C}_{l}$ a function satisfying $f(h g)=f(h) \chi(g)$ for $h \in H, g \in G$, where $\chi$ is a linear character of $G$. Then, if $X$ is a set of coset representatives of $G$ in $H$,

$$
\operatorname{det}\left(f\left(x y^{-1}\right)\right)_{x, y \in X}=\prod_{\psi \mid \chi}\left(\frac{1}{|G|} \sum_{h \in H} \psi\left(h^{-1}\right) f(h)\right)
$$

where the product runs over all linear characters $\psi$ of $H$ extending $\chi$. 
Proof. Consider the $\mathbb{C}_{l}$-linear map on $\mathbb{C}_{l} H e_{\chi}$ given by multiplication by $\tilde{f}=$ $\sum_{x \in X} f(x) x^{-1}$. With respect to the basis $\left\{x^{-1} e_{\chi}: x \in X\right\}$ it has matrix $\left(f\left(x y^{-1}\right)_{x, y \in X}\right)$; with respect to the basis $\left\{e_{\psi}: \psi \mid \chi\right\}$ it has diagonal matrix with diagonal entries $\sum_{x \in X} f(x) \psi\left(x^{-1}\right)=\frac{1}{|G|} \sum_{h \in H} f(h) \psi\left(h^{-1}\right)$ since $f(h g)=$ $f(h) \chi(g)$.

Theorem 14. Let $K / k$ be an extension of real subfields of $\mathbb{Q}\left(\zeta_{n}\right)$ with cyclic Galois group $G$ of order prime to l. If $\chi$ is a non-trivial linear $\mathbb{C}_{l}$-character of $G$, then

$$
\ell\left(\left(\mathfrak{o}_{l} \otimes_{\mathbb{Z}_{l}} \operatorname{coker} \alpha \underline{\varphi}\right)^{\chi}\right)=L_{l}(1, \chi) \prod_{\psi \mid \chi}\left(2 \prod_{p_{i} \nmid f_{\psi}}\left(\Phi\left(p_{i}^{e_{i}}\right)+1-\check{\psi}\left(p_{i}\right)\right)\right)
$$

with $\alpha$ and $\underline{\varphi}$ as in (3) of $\S 11$.

Proof. We begin by composing $\alpha \underline{\varphi}$ with the $l$-adic logarithm $\log =\prod_{\mathfrak{P} \mid l} \log _{\mathfrak{P}}$ to get

$$
\mathbb{Z}_{l} \otimes \Delta S \stackrel{\varphi}{\rightarrow} \mathbb{Z}_{l} \otimes E \stackrel{\alpha}{\rightarrow} \prod_{\mathfrak{P} \mid l} U_{\mathfrak{P}}^{1} \stackrel{\log }{\longrightarrow} \prod_{\mathfrak{P} \mid l} K_{\mathfrak{P}}
$$

This permits us to use $\mathfrak{o}_{l}$-lattice indices on the space $F_{l} \otimes_{\mathbb{Q}_{l}} \prod_{\mathfrak{P} \mid l} K_{\mathfrak{P}}$ (which are defined for arbitrary pairs of lattices on the same space). We prove

$$
\begin{aligned}
& \ell\left(\left(\mathfrak{o}_{l} \otimes_{\mathbb{Z}_{l}} \operatorname{coker}(\alpha \underline{\varphi})\right)^{\chi}\right) \\
& \quad=\left[\left(\mathfrak{o}_{l} \otimes_{\mathbb{Z}_{l}} \prod_{\mathfrak{P} \mid l} \mathfrak{O}_{\mathfrak{P}}\right)^{\chi}:\left(\mathfrak{o}_{l} \otimes_{\mathbb{Z}_{l}} \operatorname{im}(\log \alpha \underline{\varphi})\right)^{\chi}\right] / \ell\left(\left(\mathfrak{o}_{l} \otimes_{\mathbb{Z}_{l}} \prod_{\mathfrak{P} \mid l} \bar{K}_{\mathfrak{P}}\right)^{\chi}\right),
\end{aligned}
$$

where $\mathfrak{O}_{\mathfrak{P}}$ and $\bar{K}_{\mathfrak{P}}$ are the ring of integers and the residue field of $K_{\mathfrak{P}}$, respectively.

To that effect we work in $K_{0} T\left(\mathfrak{o}_{l}\right)$ and choose a sufficiently large natural integer $t$ so that $\left(\mathfrak{o}_{l} \otimes_{\mathbb{Z}_{l}} \operatorname{im}(\alpha \underline{\varphi})\right)^{\chi} \supset\left(\mathfrak{o}_{l} \otimes_{\mathbb{Z}_{l}} \prod_{\mathfrak{P} \mid l}\left(1+\mathfrak{P}^{t}\right)\right)^{\chi}$ and so that $\log _{\mathfrak{P}}: 1+\mathfrak{P}^{t} \rightarrow \mathfrak{P}^{t}$ is an isomorphism. Then

$$
\left[\left(\mathfrak{o}_{l} \otimes_{\mathbb{Z}_{l}} \operatorname{coker}(\alpha \underline{\varphi})\right)^{\chi}\right]=\left[\left(\mathfrak{o}_{l} \otimes_{\mathbb{Z}_{l}} \prod U_{\mathfrak{P}}^{1} / 1+\mathfrak{P}^{t}\right)^{\chi}\right]-\left[\left(\mathfrak{o}_{l} \otimes_{\mathbb{Z}_{l}} \frac{\operatorname{im}(\alpha \underline{\varphi})}{\prod\left(1+\mathfrak{P}^{t}\right)}\right)^{\chi}\right] .
$$

Since $\operatorname{im}(\alpha \underline{\varphi}) \simeq \mathbb{Z}_{l} \otimes \Delta S$ has no torsion,

$$
\left[\left(\mathfrak{o}_{l} \otimes_{\mathbb{Z}_{l}} \operatorname{im}(\alpha \underline{\varphi}) / \prod\left(1+\mathfrak{P}^{t}\right)\right)^{\chi}\right]=\left[\left(\mathfrak{o}_{l} \otimes_{\mathbb{Z}_{l}} \operatorname{im}(\log \alpha \underline{\varphi}) / \prod \mathfrak{P}^{t}\right)^{\chi}\right],
$$

while $\left[\left(\mathfrak{o}_{l} \otimes_{\mathbb{Z}_{l}} \prod U_{\mathfrak{P}}^{1} / 1+\mathfrak{P}^{t}\right)^{\chi}\right]=\left[\left(\mathfrak{o}_{l} \otimes_{\mathbb{Z}_{l}} \prod \mathfrak{O}_{\mathfrak{P}} / \mathfrak{P}^{t}\right)^{\chi}\right]-\left[\left(\mathfrak{o}_{l} \otimes_{\mathbb{Z}_{l}} \prod \mathfrak{O}_{\mathfrak{P}} / \mathfrak{P}\right)^{\chi}\right]$ in $K_{0} T\left(\mathfrak{o}_{l}\right)$ follows from

$$
\begin{aligned}
{\left[U_{\mathfrak{P}}^{1} / 1+\mathfrak{P}^{t}\right] } & =\sum_{i=1}^{t-1}\left[1+\mathfrak{P}^{i} / 1+\mathfrak{P}^{i+1}\right]=\sum_{i=1}^{t-1}\left[\mathfrak{P}^{i} / \mathfrak{P}^{i+1}\right] \\
& =\left[\mathfrak{P} / \mathfrak{P}^{t}\right]=\left[\mathfrak{O}_{\mathfrak{P}} / \mathfrak{P}^{t}\right]-\left[\mathfrak{O}_{\mathfrak{P}} / \mathfrak{P}\right]
\end{aligned}
$$


in $K_{0} T\left(\mathbb{Z}_{l} G_{\mathfrak{P}}\right)$ by inducing to $G$ and taking eigenspaces. Combining these two equations yields

$$
\begin{aligned}
& {\left[\left(\mathfrak{o}_{l} \otimes_{\mathbb{Z}_{l}} \operatorname{coker}(\alpha \underline{\varphi})\right)^{\chi}\right]} \\
& \quad=\left[\left(\mathfrak{o}_{l} \otimes_{\mathbb{Z}_{l}} \frac{\prod \mathfrak{O}_{\mathfrak{P}}}{\prod \mathfrak{P}^{t}}\right)^{\chi}\right]-\left[\left(\mathfrak{o}_{l} \otimes_{\mathbb{Z}_{l}} \frac{\operatorname{im}(\log \alpha \underline{\varphi})}{\prod \mathfrak{P}^{t}}\right)^{\chi}\right]-\left[\left(\mathfrak{o}_{l} \otimes_{\mathbb{Z}_{l}} \prod \bar{K}_{\mathfrak{P}}\right)^{\chi}\right],
\end{aligned}
$$

from which (1) follows by taking lengths.

The elements $e_{\chi}(1 \otimes \mathfrak{p}) \in\left(\mathfrak{o}_{l} \otimes \mathbb{Z} S\right)^{\chi}=\left(\mathfrak{o}_{l} \otimes \Delta S\right)^{\chi}, \mathfrak{p} \in S_{*}$, form an $\mathfrak{o}_{l}$-basis of $\left(\mathfrak{o}_{l} \otimes \Delta S\right)^{\chi}$. We write $(\log \alpha \underline{\varphi}) e_{\chi}(1 \otimes \mathfrak{p})$ as an $F_{l}$-linear combination of a given $\mathfrak{o}_{l}$-basis $v_{1}, \ldots, v_{[k: \mathbb{Q}]}$ of $\left(\mathfrak{o}_{l} \otimes_{\mathbb{Z}_{l}} \bar{\prod} \mathfrak{O}_{\mathfrak{P}}\right)^{\chi}$ :

$$
(\log \alpha \underline{\varphi})\left(e_{\chi}(1 \otimes \mathfrak{p})\right)=\sum_{i=1}^{[k: \mathbb{Q}]} a_{i, \mathfrak{p}} v_{i}, \mathfrak{p} \in S_{*} .
$$

Thus

$$
\left[\left(\mathfrak{o}_{l} \otimes_{\mathbb{Z}_{l}} \prod_{\mathfrak{P} \mid l} \mathfrak{O}_{\mathfrak{P}}\right)^{\chi}:\left(\mathfrak{o}_{l} \otimes_{\mathbb{Z}_{l}} \operatorname{im}(\log \alpha \underline{\varphi})\right)^{\chi}\right]=\operatorname{det}\left(a_{i, \mathfrak{p}}\right) \mathfrak{o}_{l} .
$$

The group $G$ acts on the right on the set of $\mathbb{Q}$-embeddings of $K$ into $\mathbb{C}_{l}$. Let $\left\{s: K \rightarrow \mathbb{C}_{l}\right\}$ be a set of orbit representatives. Each $s$ extends to an $F_{l}$-algebra homomorphism

$$
F_{l} \otimes_{\mathbb{Q}_{l}} \prod_{\mathfrak{P} \mid l} K_{\mathfrak{P}}=F_{l} \otimes_{\mathbb{Q}_{l}}\left(\mathbb{Q}_{l} \otimes_{\mathbb{Q}} K\right)=F_{l} \otimes_{\mathbb{Q}} K \stackrel{s}{\rightarrow} \mathbb{C}_{l},
$$

which when applied to (a) gives the equation

$$
\left(s(\log \alpha \underline{\varphi})\left(e_{\chi}(1 \otimes \mathfrak{p})\right)\right)_{\mathfrak{p}, s}=\left(a_{i, \mathfrak{p}}\right)_{\mathfrak{p}, i}\left(s v_{i}\right)_{i, s}
$$

of matrices over $\mathbb{C}_{l}$. Taking determinants yields

$$
\operatorname{det}\left(a_{i, \mathfrak{p}}\right)=\operatorname{det}\left(s(\log \alpha \underline{\varphi})\left(e_{\chi}(1 \otimes \mathfrak{p})\right)\right) / \operatorname{det}\left(s v_{i}\right) .
$$

We first turn to the numerator and prove

$$
\begin{aligned}
& \operatorname{det}\left(s(\log \alpha \underline{\varphi})\left(e_{\chi}(1 \otimes \mathfrak{p})\right)\right)_{s, \mathfrak{p}} \\
& \quad \sim_{l} \prod_{\psi \mid \chi}\left[2 \tau_{l}(\psi) L_{l}(1, \psi)\left(1-\frac{\psi(l)}{l}\right)^{-1} \prod_{p_{i} \nmid f_{\psi}}\left(\Phi\left(p_{i}^{e_{i}}\right)+1-\check{\psi}\left(p_{i}\right)\right)\right],
\end{aligned}
$$

where $\sim_{l}$ means equality up to $l$-units in $\mathbb{C}_{l}$ and $\tau_{l}(\chi)$ is the "l-adic Gauss sum" of the $l$-adic character $\chi$. Namely, if $\chi=i_{l}\left(\chi^{\prime}\right)$ for an $F$-character $\chi^{\prime}$ and an embedding $i_{l}: F \rightarrow \mathbb{C}_{l}$, then $\tau_{l}(\chi)$ is defined to be $i_{l}\left(\tau\left(\chi^{\prime}\right)\right)$ with $\tau\left(\chi^{\prime}\right)$ denoting the usual Galois Gauss sum [Ma, p.48]. Changing the embedding varies $\chi^{\prime}$ by Galois conjugation over $\mathbb{Q}$ and so, by a theorem of Fröhlich [Ma, p.50], $\tau_{l}(\chi)$ is well-defined up to multiplication by a root of unity, which is irrelevant in (4).

To prove (4) we define $X$ to be the set of coset representatives of $G$ in $H=$ $G(K / \mathbb{Q})$ such that $S_{*}=\{x \infty: x \in X\}$. Fix an embedding $s_{1}$ in the set of all 
$\mathbb{Q}$-embeddings of $K$ into $\mathbb{C}_{l}$ and choose $\left\{s_{1} y^{-1}: y \in X\right\}$ as our set $\left\{s: K \rightarrow \mathbb{C}_{l}\right\}$ of $G$-orbit representatives. With $\mathfrak{p}=x \infty$ and $s=s_{1} y^{-1}$ we have

$$
\begin{gathered}
s(\log \alpha \underline{\varphi})\left(e_{\chi}(1 \otimes \mathfrak{p})\right)=s(\log \alpha)\left(\frac{1}{|G|} \sum_{g \in G} \chi\left(g^{-1}\right) \otimes \xi_{K}^{g x-1} / \xi_{K}^{x-1}\right) \\
=\frac{1}{|G|} s_{1} y^{-1}\left(\sum_{g \in G} \chi\left(g^{-1}\right) \otimes \log \xi_{K}^{(g-1) x}\right) \\
=\frac{1}{|G|} \sum_{g \in G} \chi\left(g^{-1}\right) s_{1} \log \left(\xi_{K}^{x y^{-1}(g-1)}\right) .
\end{gathered}
$$

On writing $s_{1} \log =\log _{l} s_{1}$ with $\log _{l}: \mathbb{C}_{l}^{\times} \rightarrow \mathbb{C}_{l}$ defined as in [Wa, p.50] the above is $\frac{1}{|G|} \sum_{g \in G} \chi\left(g^{-1}\right) \log _{l} s_{1}\left(\xi_{K}^{x y^{-1} g}\right)$ because $\chi \neq 1$. With $f(h)=\frac{1}{|G|} \sum_{g \in G} \chi\left(g^{-1}\right) \times$ $\log _{l} s_{1}\left(\xi_{K}^{h g}\right)$ we obtain from Lemma 13

$$
\begin{aligned}
\operatorname{det}( & \left.(\log \alpha \underline{\varphi})\left(e_{\chi}(1 \otimes \mathfrak{p})\right)\right)_{s, \mathfrak{p}} \\
& =\prod_{\psi \mid \chi}\left[\frac{1}{|G|^{2}} \sum_{h \in H} \psi\left(h^{-1}\right) \sum_{g \in G} \chi\left(g^{-1}\right) \log _{l} s_{1}\left(\xi_{K}^{h g}\right)\right] \\
& =\prod_{\psi \mid \chi}\left[\frac{1}{|G|} \sum_{h \in H} \psi\left(h^{-1}\right) \log _{l} s_{1}\left(\xi_{K}^{h}\right)\right]
\end{aligned}
$$

by substituting $h^{-1} g$ for $h$. Let $\zeta=\zeta_{n}$. Since $K$ is real, $\xi_{K}=N_{\mathbb{Q}(\zeta) / K}\left(\prod_{I}\left(1-\zeta^{n_{I}}\right)\right)^{2}$ and so, inflating $\psi$ to $G(\mathbb{Q}(\zeta) / \mathbb{Q})$, the above $\psi$-term equals

$$
\begin{aligned}
\frac{1}{|G|} & \sum_{h \in G(\mathbb{Q}(\zeta) / \mathbb{Q})} \check{\psi}(h) \log _{l} s_{1}\left(\prod_{I}\left(1-\zeta^{n_{I}}\right)^{h}\right)^{2} \\
& \sim_{l} 2 \sum_{a \in(\mathbb{Z} / n)^{\times}} \check{\psi}(a) \log _{l}\left(\prod_{I}\left(1-\left(s_{1} \zeta\right)^{a n_{I}}\right)\right) .
\end{aligned}
$$

By the $l$-adic class number formula [Wa, p.63] and the calculation given at the end of p. 152 and the beginning of p. 153 in [Wa] we get that our determinant

$$
\sim_{l} \prod_{\psi \mid \chi}\left[2 \tau_{l}(\psi) L_{l}(1, \psi)\left(1-\frac{\psi(l)}{l}\right)^{-1} \prod_{p_{i} \nmid f_{\psi}}\left(\Phi\left(p_{i}^{e_{i}}\right)+1-\check{\psi}\left(p_{i}\right)\right)\right]
$$

where we have replaced Washington's Gauss sum $\tau_{w}(\check{\psi})$ by $\tau_{l}(\psi)$. These agree by the functional equation; compare [Wa, p.29] and [Ma, p.48].

We now turn to the denominator in (3) and show

$$
\operatorname{det}\left(s v_{i}\right)_{s, i} \sim_{l} \prod_{\mathfrak{q} \mid l} d_{k_{\mathfrak{q}} / \mathbb{Q}_{l}}^{1 / 2} \tau_{l}\left(\chi_{\mathfrak{q}}\right)
$$

where $d_{k_{\mathfrak{q}} / \mathbb{Q}_{l}}$ is the discriminant of $k_{\mathfrak{q}} / \mathbb{Q}_{l}$ and where $\chi_{\mathfrak{q}}$ is the restriction of $\chi$ to a decomposition group $G_{\mathfrak{Q}}$ of $\mathfrak{q}$. 
In order to prove (5) we choose the basis $\left\{v_{i}\right\}$ according to the decomposition

$$
\begin{aligned}
\left(\mathfrak{o}_{l} \otimes_{\mathbb{Z}_{l}} \prod_{\mathfrak{P} \mid l} \mathfrak{O}_{\mathfrak{P}}\right)^{\chi}=\left(\mathfrak{o}_{l} \otimes_{\mathbb{Z}_{l}} \prod_{\mathfrak{q} \mid l} \prod_{\mathfrak{P} \mid \mathfrak{q}} \mathfrak{O}_{\mathfrak{P}}\right)^{\chi} \\
\quad=\prod_{\mathfrak{q} \mid l}\left(\mathfrak{o}_{l} \otimes_{\mathbb{Z}_{l}} \prod_{\mathfrak{P} \mid \mathfrak{q}} \mathfrak{O}_{\mathfrak{P}}\right)^{\chi}=\prod_{\mathfrak{q} \mid l}\left(\mathfrak{o}_{l} \otimes_{\mathbb{Z}_{l}} \mathfrak{O}_{\mathfrak{Q}}\right)^{\chi_{\mathfrak{q}}},
\end{aligned}
$$

where $\mathfrak{Q}$ denotes a fixed prime of $K$ above $\mathfrak{q}$. The last equation is Frobenius reciprocity from $\prod_{\mathfrak{P} \mid \mathfrak{q}} \mathfrak{O}_{\mathfrak{P}}=\operatorname{ind}_{G_{\mathfrak{Q}}}^{G}\left(\mathfrak{O}_{\mathfrak{Q}}\right)$.

We now put special $\mathfrak{o}_{l}$-bases $\left\{v_{i_{\mathfrak{q}}}^{(\mathfrak{q})}: 1 \leq i_{\mathfrak{q}} \leq\left[k_{\mathfrak{q}}: \mathbb{Q}_{l}\right]\right\}$ of $\left(\mathfrak{o}_{l} \otimes_{\mathbb{Z}_{l}} \mathfrak{O}_{\mathfrak{Q}}\right)^{\chi_{\mathfrak{q}}}$ together in order to arrive at a basis $\left\{v_{i}\right\}$ which will be appropriate for proving (5). Since $l \nmid|G|$, the extension $K / k$ is tame and so $\mathfrak{O}_{\mathfrak{Q}}$ free over $\mathfrak{o}_{\mathfrak{q}} G_{\mathfrak{Q}}$ (with $\mathfrak{o}_{\mathfrak{q}}$ denoting the ring of integers in $k_{\mathfrak{q}}$ ). Hence if $a_{\mathfrak{Q}} \in \mathfrak{O}_{\mathfrak{Q}}$ generates an integral normal basis, then $\left(\mathfrak{o}_{l} \otimes_{\mathbb{Z}_{l}} \mathfrak{O}_{\mathfrak{Q}}\right)^{\chi_{\mathfrak{q}}}$ is free on $e_{\chi_{\mathfrak{q}}}\left(1 \otimes a_{\mathfrak{Q}}\right)$ over $\mathfrak{o}_{l} \otimes_{\mathbb{Z}_{l}} \mathfrak{o}_{\mathfrak{q}}$. We set $v_{i_{\mathfrak{q}}}^{(\mathfrak{q})}=\left(1 \otimes u_{i_{\mathfrak{q}}}^{(\mathfrak{q})}\right) e_{\chi_{\mathfrak{q}}}\left(1 \otimes a_{\mathfrak{Q}}\right)$ with $\left\{u_{i_{\mathfrak{q}}}^{(\mathfrak{q})}: 1 \leq i_{\mathfrak{q}} \leq\left[k_{\mathfrak{q}}: \mathbb{Q}_{l}\right]\right\}$ a $\mathbb{Z}_{l}$-basis of $\mathfrak{o}_{\mathfrak{q}}$.

We also work with a well-chosen set $\left\{s: K \rightarrow \mathbb{C}_{l}\right\}$. We are allowed to do so because (3) is independent of the choice of embeddings and (4) is changed by the root of unity $\prod_{s} \chi\left(g_{s}\right)$, if $s$ is replaced by $s g_{s}$ with $g_{s} \in G$, since $\left(s g_{s}\right)(\log \alpha \varphi) e_{\chi}=$ $s(\log \alpha \underline{\varphi}) g_{s} e_{\chi}=\chi\left(g_{s}\right) s(\log \alpha \underline{\varphi}) e_{\chi}$.

For each $\mathfrak{q} \mid l$ fix an embedding $s_{\mathfrak{Q}}: K \rightarrow \mathbb{C}_{l}$ inducing our $\mathfrak{Q}$ on $K$ and a set $X_{\mathfrak{q}}$ of left coset representatives of $G_{s_{\mathbb{Q}_{2}} k_{\mathrm{q}}}$ in $G_{\mathbb{Q}_{l}}$ (with $G_{L}$, for a field $L$, denoting the absolute Galois group of $L)$. Then our set $\left\{s: K \rightarrow \mathbb{C}_{l}\right\}$ shall be $\bigcup_{\mathfrak{q} \mid l}\left\{\gamma_{\mathfrak{q}} s_{\mathfrak{Q}}: \gamma_{\mathfrak{q}} \in\right.$ $\left.X_{\mathfrak{q}}\right\}$. Here the extended $F_{l}$-algebra homomorphism $s_{\mathfrak{Q}}: F_{l} \otimes_{\mathbb{Q}} \prod_{\mathfrak{P} \mid l} K_{\mathfrak{P}} \rightarrow \mathbb{C}_{l}$ has $s_{\mathfrak{Q}^{\prime}}\left(v_{i_{\mathfrak{q}}}^{(\mathfrak{q})}\right)=0$ if $\mathfrak{q}^{\prime} \neq \mathfrak{q}$. Thus we obtain

$$
\operatorname{det}\left(s v_{i}\right)_{s, i}=\prod_{\mathfrak{q} \mid l} \operatorname{det}\left(\gamma_{\mathfrak{q}} s_{\mathfrak{Q}} v_{i_{\mathfrak{q}}}^{(\mathfrak{q})}\right)_{\gamma_{\mathfrak{q}}, i_{\mathfrak{q}}} .
$$

For (5) it therefore suffices to show $\operatorname{det}\left(\gamma_{\mathfrak{q}} s_{\mathfrak{Q}} v_{i_{\mathfrak{q}}}^{(\mathfrak{q})}\right)_{\gamma_{\mathfrak{q}}, i_{\mathfrak{q}}} \sim_{l} d_{k_{\mathfrak{q}} / \mathbb{Q}_{l}}^{1 / 2} \tau_{l}\left(\chi_{\mathfrak{q}}\right)$ for each $\mathfrak{q}$. Now

$$
\gamma_{\mathfrak{q}} s_{\mathfrak{Q}} v_{i_{\mathfrak{q}}}^{(\mathfrak{q})}=\gamma_{\mathfrak{q}} s_{\mathfrak{Q}} u_{i_{\mathfrak{q}}}^{(\mathfrak{q})} \cdot \frac{1}{\left|G_{\mathfrak{Q}}\right|} \sum_{g \in G_{\mathfrak{Q}}} \chi_{\mathfrak{q}}\left(g^{-1}\right)\left(\gamma_{\mathfrak{q}} s_{\mathfrak{Q}}\right)\left(g a_{\mathfrak{Q}}\right)
$$

and so

$$
\operatorname{det}\left(\gamma_{\mathfrak{q}} s_{\mathfrak{Q}} v_{i_{\mathfrak{q}}}^{(\mathfrak{q})}\right) \sim_{l} \operatorname{det}\left(\gamma_{\mathfrak{q}} s_{\mathfrak{Q}} u_{i_{\mathfrak{q}}}^{(\mathfrak{q})}\right) \prod_{\gamma_{\mathfrak{q}} \in X_{\mathfrak{q}}}\left(\sum_{g \in G_{\mathfrak{Q}}} \chi_{\mathfrak{q}}\left(g^{-1}\right)\left(\gamma_{\mathfrak{q}} s_{\mathfrak{Q}}\right)\left(g a_{\mathfrak{Q}}\right)\right) .
$$

The first factor is $d_{s_{\mathfrak{Q}} k_{\mathfrak{q}} / \mathbb{Q}_{l}}^{1 / 2}=d_{k_{\mathfrak{q}} / \mathbb{Q}_{l}}^{1 / 2}$.

To deal with the second factor we do transport of structure via $s_{\mathfrak{Q}}$. The natural identification $g \longleftrightarrow \gamma$ of $G_{\mathfrak{Q}}=G\left(K_{\mathfrak{Q}} / k_{\mathfrak{q}}\right)$ with $\Gamma_{\mathfrak{Q}}=G\left(s_{\mathfrak{Q}} K_{\mathfrak{Q}} / s_{\mathfrak{Q}} k_{\mathfrak{q}}\right)$ satisfies $s_{\mathfrak{Q}} g=\gamma_{\mathfrak{Q}}$; it defines $\chi_{\mathfrak{q}}^{\prime}$ on $\Gamma_{\mathfrak{Q}}$ by $\chi_{\mathfrak{q}}^{\prime}(\gamma)=\chi_{\mathfrak{q}}(g)$. Hence

$$
\begin{aligned}
& \sum_{g \in G_{\mathfrak{Q}}} \chi_{\mathfrak{q}}\left(g^{-1}\right)\left(\gamma_{\mathfrak{q}} s_{\mathfrak{Q}}\right)\left(g a_{\mathfrak{Q}}\right)=\sum_{\gamma \in \Gamma_{\mathfrak{Q}}} \chi_{\mathfrak{q}}^{\prime}\left(\gamma^{-1}\right)\left(\gamma_{\mathfrak{q}} \gamma s_{\mathfrak{Q}}\right)\left(a_{\mathfrak{Q}}\right) \\
& =\gamma_{\mathfrak{q}}\left(\sum_{\gamma}\left(\gamma_{\mathfrak{q}}^{-1} \chi_{\mathfrak{q}}^{\prime}\right)\left(\gamma^{-1}\right) \gamma\left(s_{\mathfrak{Q}} a_{\mathfrak{Q}}\right)\right)=\gamma_{\mathfrak{q}}\left(\left.s_{\mathfrak{Q}} a_{\mathfrak{Q}}\right|^{\gamma_{\mathfrak{q}}^{-1}} \chi_{\mathfrak{q}}^{\prime}\right)
\end{aligned}
$$


where $(\cdot \mid \cdot)$ is the resolvent of $[\mathrm{Fr} 1, \mathrm{p} .29] .{ }^{4}$ Taking the product over $\gamma_{\mathfrak{q}} \in X_{\mathfrak{q}}$ gives the norm resolvent $\mathcal{N}_{s_{\mathfrak{Q}} k_{\mathfrak{q}} / \mathbb{Q}_{l}}\left(s_{\mathfrak{Q}} a_{\mathfrak{Q}} \mid \chi_{\mathfrak{q}}^{\prime}\right)\left[\right.$ Fr1, p.31] and this, in turn, is $\sim_{l} \tau_{l}\left(\chi_{\mathfrak{q}}^{\prime}\right) \sim_{l}$ $\tau_{l}\left(\chi_{\mathfrak{q}}\right)$ by [Fr1, p.127, Theorem 23]. This proves (5).

In order to complete the proof of our theorem we finally show

$$
\begin{gathered}
\prod_{\psi \mid \chi} \tau_{l}(\psi) \sim_{l} d_{k / \mathbb{Q}}^{1 / 2} \tau_{l}(\chi) \sim_{l} \prod_{\mathfrak{q} \mid l} d_{k_{\mathfrak{q}} / \mathbb{Q}_{l}}^{1 / 2} \tau_{l}\left(\chi_{\mathfrak{q}}\right), \\
L_{l}(1, \chi)=\prod_{\psi \mid \chi} L_{l}(1, \psi), \\
\prod_{\psi \mid \chi}\left(1-\frac{\psi(l)}{l}\right)^{-1} \sim_{l} \ell\left(\left(\mathfrak{o}_{l} \otimes_{\mathbb{Z}_{l}} \prod_{\mathfrak{P} \mid l} \bar{K}_{\mathfrak{P}}\right)^{\chi}\right) .
\end{gathered}
$$

Together with (4) these yield

$$
\begin{aligned}
& \operatorname{det}\left(s(\log \alpha \underline{\varphi})\left(e_{\chi}(1 \otimes \mathfrak{p})\right)\right)_{s, \mathfrak{p}} \\
& \quad \sim_{l} \tau_{l}(\chi) d_{k / \mathbb{Q}}^{1 / 2} L_{l}(1, \chi) \ell\left(\left(\mathfrak{o}_{l} \otimes_{\mathbb{Z}_{l}} \prod_{\mathfrak{P} \mid l} \bar{K}_{\mathfrak{P}}\right)^{\chi}\right) \prod_{\psi \mid \chi}\left(2 \prod_{p_{i} \nmid f_{\psi}}\left(\Phi\left(p_{i}^{e_{i}}\right)+1-\check{\psi}\left(p_{i}\right)\right)\right) .
\end{aligned}
$$

This multiplied with the inverse of the right hand side of (5) becomes

$$
\operatorname{det}\left(a_{i, \mathfrak{p}}\right) \sim_{l} L_{l}(1, \chi) \ell\left(\left(\mathfrak{o}_{l} \otimes_{\mathbb{Z}_{l}} \prod_{\mathfrak{P} \mid l} \bar{K}_{\mathfrak{P}}\right)^{\chi}\right) \prod_{\psi \mid \chi}\left(2 \prod_{p_{i} \nmid f_{\psi}}\left(\Phi\left(p_{i}^{e_{i}}\right)+1-\check{\psi}\left(p_{i}\right)\right)\right),
$$

by (3). Then (1) and (2) finish the proof of Theorem 14 .

Proof of (6). From [Ma, p.48] (where $W_{\infty}(\tilde{\psi})$ is a unit) we get

$$
\begin{aligned}
& \prod_{\psi \mid \chi} \tau_{l}(\psi) \sim_{l}\left(\prod_{\psi \mid \chi} W(\check{\psi}) f_{\psi}^{1 / 2}\right)=W\left(\operatorname{ind}_{G}^{G(K / \mathbb{Q})} \check{\chi}\right)\left(f_{\operatorname{ind}_{G}^{G(K / \mathbb{Q})} \chi}\right)^{1 / 2} \\
& =W(\check{\chi}) N_{k / \mathbb{Q}}\left(f_{\chi}\right)^{1 / 2} d_{k / \mathbb{Q}}^{1 / 2} \sim_{l} \tau_{l}(\chi) d_{k / \mathbb{Q}}^{1 / 2} \quad[\text { Ma, pp.18,22-23]. }
\end{aligned}
$$

The discriminant part of the second relation is standard while the Gauss sum part follows from

$$
\tau(\chi)=\prod_{\text {finite } \mathfrak{q}} \tau\left(\chi_{\mathfrak{q}}\right) \quad[\mathrm{Ma}, \mathrm{p} .49] \quad \text { and } \quad \tau\left(\chi_{\mathfrak{q}}\right) \sim_{l} 1 \text { for } \mathfrak{q} \nmid l \quad[\mathrm{Ma}, \mathrm{p} .39] .
$$

Proof of (7). By $[\mathrm{Gr} 2, \mathrm{p} .81]$ we have $L_{l}(s, \chi)=L_{l}\left(s, \operatorname{ind}_{G}^{G(K / \mathbb{Q})}(\chi)\right)=\prod_{\psi \mid \chi} L_{l}(s, \psi)$.

Proof of (8). For $\mathfrak{P}|\mathfrak{q}| l$ let $e$ and $f$ denote the absolute ramification index and residue degree of $\mathfrak{q}$, respectively, and $I$ the inertia group of $\mathfrak{P} \mid \mathfrak{q}$. Also, let $\bar{k}_{\mathfrak{q}}$ be the residue field of $k$ at $\mathfrak{q}$. Then

$$
\prod_{\mathfrak{P} \mid l} \bar{K}_{\mathfrak{P}}=\prod_{\mathfrak{q} \mid l} \prod_{\mathfrak{P} \mid \mathfrak{q}} \bar{K}_{\mathfrak{P}}=\prod_{\mathfrak{q} \mid l} \operatorname{ind}_{I}^{G}\left(\bar{k}_{\mathfrak{q}}\right)=\prod_{\mathfrak{q} \mid l} \operatorname{ind}_{I}^{G}\left(\mathbb{F}_{l}^{f}\right)=\left(\operatorname{ind}_{I}^{G} \mathbb{F}_{l}\right)^{[k: \mathbb{Q}] / e}
$$

and $\mathfrak{o}_{l} \otimes_{\mathbb{Z}_{l}} \prod_{\mathfrak{P} \mid l} \bar{K}_{\mathfrak{P}}=\left(\operatorname{ind}_{I}^{G}\left(\mathfrak{o}_{l} / l \mathfrak{o}_{l}\right)\right)^{[k: \mathbb{Q}] / e}$. Since $l \nmid|G|$, the $\chi$-eigenspace has length

$$
l^{\left(\chi, \frac{[k: \mathbb{Q}]}{e} \operatorname{ind}_{I}^{G}(1)\right)} \mathfrak{o}_{l}= \begin{cases}l^{[k: \mathbb{Q}] / e} \mathfrak{o}_{l} & \text { if } \chi \mid I=1, \\ 1 & \text { else }\end{cases}
$$

\footnotetext{
${ }^{4}$ Our Galois action is on the left.
} 
On the other hand, $\prod_{\psi \mid \chi}\left(1-\frac{\psi(l)}{l}\right)^{-1}=\prod_{\substack{\psi \mid \chi \\ l \nmid f \psi}} \frac{l}{l-\psi(l)} \sim_{l} l^{b} \quad$ with $b$ counting the $\psi$ above $\chi$ whose conductor is coprime to $l$, hence $\psi(l)$ is a root of unity. Look now at the diagram below in which $I^{\prime}$ is the inertia group of $\mathfrak{P} \mid l$, so $I=I^{\prime} \cap G$.

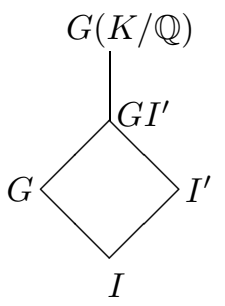

By [Se, pp.109,111], $f_{\psi} \not \equiv 0 \bmod l$ if, and only if, $\psi \mid I^{\prime}=1$. Of course, there are $\left[G(k / \mathbb{Q}): G I^{\prime}\right]=$ $[k: \mathbb{Q}] / e$ many of these if $\chi \mid I=1$, otherwise there are none.

\section{The CONNECTION to IwASAwa theory}

This section pieces together facts from the literature which yield

Theorem 15. Let $l$ be a prime, $K / k$ a cyclic extension of real fields of degree prime to $l$, and $M$ the maximal abelian l-extension of $K$ which is unramified outside $l$. If $l=2$ assume that $K / \mathbb{Q}$ is abelian and not ramified at 2 . Then, if $\chi$ is a non-trivial faithful linear $\mathbb{C}_{l}$-character of $G=G(K / k)$,

$$
\ell\left(\left(\mathfrak{o}_{l} \otimes_{\mathbb{Z}_{l}} G(M / K)\right)^{\chi}\right)=2^{-[k: \mathbb{Q}]} L_{l}(1, \chi) \mathfrak{o}_{l},
$$

where $\mathfrak{o}_{l}$ is unramified over $\mathbb{Z}_{l}$ and large enough to contain the values of all $\mathbb{C}_{l}$ characters of $G$.

In the proof of the theorem we shall have to look at characteristic polynomials of finitely generated modules over the Iwasawa algebra $\Lambda=\mathfrak{o}_{l}[[T]]$ [Wa, p.114], [Ko, p.12]. If $f, g \in \Lambda$, then the notation $f \sim g$ indicates an equality $f=g h$ with a unit $h \in \Lambda$. With respect to $\sim$ we can write each characteristic polynomial as a product $l^{m} G^{*}(T)$ where $m \geq 0$ and $G^{*}(T)$ is a distinguished polynomial [Wa, $\S 7.1$. Since $K \cap k_{\infty}=k$, as follows from $l \nmid[K: k]$ [ " $\chi$ is of type $S$ "], we have $L_{l}(1-s, \chi)=G_{\chi}\left(u^{s}-1\right)$ with $G_{\chi}(T) \in \Lambda$ [Wi1, p.494]. Here $u \in \mathbb{Z}_{l}^{\times}$satisfies $\eta^{\gamma}=\eta^{u}$ for all $l$-power roots of unity $\eta$ and a topological generator $\gamma$ for the Galois group $\Gamma=G\left(k_{\infty} / k\right)$ of the cyclotomic $\mathbb{Z}_{l}$-extension $k_{\infty}$ of $k .{ }^{5}$ The first main ingredient of our proof now is

$$
L_{l}(1-s, \chi) \sim\left\{\begin{array}{cc}
l^{m_{\chi}} G_{\chi}^{*}\left(u^{s}-1\right) & \text { if } l \neq 2 \\
2^{[k: \mathbb{Q}]} G_{\chi}^{*}\left(u^{s}-1\right) & \text { if } l=2 .
\end{array}\right.
$$

Supposedly $m_{\chi}=0$ but we will not need this. So only the case $l=2$ has to be explained. As $K / \mathbb{Q}$ is abelian, all irreducible $\mathbb{C}_{l}$-constituents $\theta$ of $\operatorname{ind}_{G}^{G(K / \mathbb{Q})}(\chi)$ are linear. They are of type $S$, since the ramification assumption on $K$ implies $K \cap \mathbb{Q}_{\infty}=\mathbb{Q}$. By the induction property of the $l$-adic $L$-series [Gr2] formula (i) for $l=2$ follows from the corresponding formula with respect to the base field $\mathbb{Q}$ which holds because of the vanishing of Iwasawa's $\mu$-invariant for $\mathbb{Q}[$ Wa, Theorem 7.15, p.130]: for this implies that the power series $f(T, \theta) \in \mathbb{Z}_{2}[[T]]$ in $L_{2}(s, \theta)=$ $f\left(u^{s}-1, \theta\right)$ of [Wa, Theorem 7.14, p.127] is precisely divisible by 2 in $\mathbb{Z}_{2}[[T]]$.

\footnotetext{
${ }^{5}$ Not to be confused with the earlier mentioned Galois group $G(F / \mathbb{Q})$ which does not show up in this section.
} 
We now turn to the case $l \neq 2$ and draw a diagram which displays the fields we have to work with.

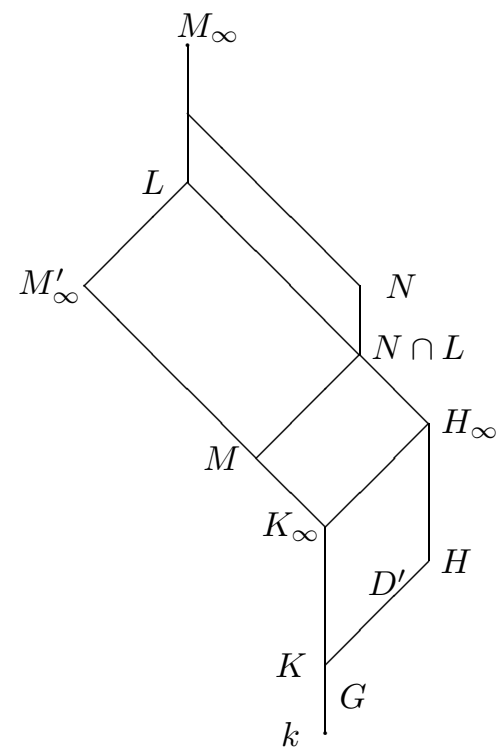

$\zeta_{l}$ is a primitive root of unity of order $l$ and $H=K\left(\zeta_{l}\right), D^{\prime}=G(H / K)$. Set $D=G(H / k)$, so $G=D / D^{\prime}$.

$K_{\infty}$ is the cyclotomic $l$-extension of $K$, $H_{\infty}=H \cdot K_{\infty}$;

$M_{\infty}^{\prime}$ is the maximal abelian $l$-ramified $l$ extension of $K_{\infty}, L=M_{\infty}^{\prime} \cdot H_{\infty}$;

$M_{\infty}$ is the maximal abelian $l$-ramified $l$ extension of $H_{\infty}, N$ the maximal abelian extension of $H$ in $M_{\infty}$ and $M=M_{\infty}^{\prime} \cap(N \cap L)$.

We first list some observations.

(1) $H_{\infty}$ is the cyclotomic l-extension of $H$.

(2) $L$ is the maximal abelian extension of $K_{\infty}$ in $M_{\infty}$. For, if $L^{\prime}$ denotes that field, then $L^{\prime} \supset L$ and $G\left(L^{\prime} / K_{\infty}\right)$ splits into the direct product of its Sylow $l$-subgroup and a subgroup isomorphic to $D^{\prime}$. The fixed field of the subgroup is $M_{\infty}^{\prime}$, as it is an $l$-ramified $l$-extension of $K_{\infty}$ containing $M_{\infty}^{\prime}$.

(3) $N \cap L$ is the maximal abelian extension of $K$ in $M_{\infty}$. This results from $N \cap L / K$ and $M_{\infty}^{\prime} / K$ being Galois extensions, so $M / K$ is one as well. Since $(|N \cap L: M|, l)=1$, the group $[G(N \cap L / H), G(N \cap L / M)]$ of commutators of elements in $G(N \cap L / H)$ and $G(N \cap L / M)$ vanishes and $N \cap L / K$ is thus abelian. On the other hand, the maximal abelian extension of $K$ in $M_{\infty}$ contains $H$ and so is contained in $N$. As it also contains $K_{\infty}$, it is a subfield of $L$ by (2).

(4) The $D^{\prime}$-invariant elements in $G(N / N \cap L)$ are trivial. Set $U=G(N / N \cap L)$. So $U$ is an abelian $l$-group and $H^{ \pm 1}\left(D^{\prime}, U\right)=0$. Consequently, $U^{D^{\prime}}=N_{D^{\prime}} U$ and ker $N_{D^{\prime}}=I_{D^{\prime}} U$ (in the obvious notation). Now, $U / I_{D^{\prime}} U$ is the Galois group of an extension $F / N \cap L, F \subset N$, which uniquely corresponds to an extension $F^{\prime} / L, F^{\prime} \subset L N \subset M_{\infty}$.

We show that $F^{\prime}$ is abelian over $K_{\infty}$. Since both $N$ and $N \cap L$ are Galois extensions of $K_{\infty}$, so is $F$ as $I_{D^{\prime}} U$ is $D^{\prime}$-stable. Moreover, $U / I_{D^{\prime}} U$ is $D^{\prime}$-trivial and consequently $F / K_{\infty}$ is abelian. Therefore $F^{\prime}=L F$ is abelian over $K_{\infty}$.

From (2) we obtain $F^{\prime}=L$ and thus $F=N \cap L$ and $I_{D^{\prime}} U=U$. So ker $N_{D^{\prime}}=U$ and $N_{D^{\prime}}=1$.

(5) $M$ is the maximal abelian extension of $K$ in $M_{\infty}^{\prime}$. By (3) it is abelian. If there was a bigger abelian extension, then its compositum with $N \cap L$ would properly contain $N \cap L$, contradicting (3). As a corollary, $M$ is indeed the maximal abelian $l$-ramified $l$-extension of $K$.

(6) Inflate $\chi$ from $G$ to $D$. Then $\mathfrak{o}_{l} \otimes_{\mathbb{Z}_{l}} G(N / N \cap L)$ is an $\mathfrak{o}_{l} D$-module satisfying $\left(\mathfrak{o}_{l} \otimes_{\mathbb{Z}_{l}} G(N / N \cap L)\right)^{\chi}=1$. Namely, $\sigma x=\chi(\sigma) x=x$ for $\sigma \in D^{\prime}$ and $x \in$ $\left(\mathfrak{o}_{l} \otimes_{\mathbb{Z}_{l}} G(N / N \cap L)\right)^{\chi}$. Apply (4). 
Set $Y=G\left(M_{\infty} / H_{\infty}\right), \bar{Y}=G\left(N \cap L / H_{\infty}\right)$ and $\Gamma=G\left(K_{\infty} / K\right)=G\left(H_{\infty} / H\right)$. Then by the definition of $N, Y_{\Gamma}=G\left(N / H_{\infty}\right)$, and by (3), $\bar{Y}_{\Gamma}=\bar{Y}$. We can identify $\bar{Y}$ and $G\left(M / K_{\infty}\right)$. Now, on tensoring the two exact sequences

$$
G(N / N \cap L) \longmapsto Y_{\Gamma} \rightarrow \bar{Y}, G\left(M / K_{\infty}\right) \longmapsto G(M / K) \rightarrow G\left(K_{\infty} / K\right)
$$

with $\mathfrak{o}_{l}$ over $\mathbb{Z}_{l}$ and then taking $\chi$-eigenspaces we arrive at

$$
\begin{aligned}
& \left(\mathfrak{o}_{l} \otimes_{\mathbb{Z}_{l}} Y_{\Gamma}\right)^{\chi}=\left(\mathfrak{o}_{l} \otimes_{\mathbb{Z}_{l}} \bar{Y}\right)^{\chi}, \text { by }(6), \\
& \left(\mathfrak{o}_{l} \otimes_{\mathbb{Z}_{l}} G(M / K)\right)^{\chi}=\left(\mathfrak{o}_{l} \otimes_{\mathbb{Z}_{l}} G\left(M / K_{\infty}\right)\right)^{\chi},
\end{aligned}
$$

since, as has already been observed in $\S 11, K_{\infty} / k$ is abelian and $\chi \neq 1$.

Remember that we are interested in $\left(\mathfrak{o}_{l} \otimes_{\mathbb{Z}_{l}} G(M / K)\right)^{\chi}$. Because of the above we may replace this group by $\left(\mathfrak{o}_{l} \otimes_{\mathbb{Z}_{l}} Y_{\Gamma}\right)^{\chi}$ and are in position to apply results of [Wi1].

By the Main Conjecture [Wi1, Theorem 1.3, p.496] $\left(\mathfrak{o}_{l} \otimes_{\mathbb{Z}_{l}} Y\right)^{\chi}$ is a finitely generated $\Lambda$-torsion module and has characteristic polynomial $l^{m_{\chi}^{\prime}} G_{\chi}^{*}(T)$ with some $m_{\chi}^{\prime} \geq 0$ and the $G_{\chi}^{*}$ from (i).

By [Gr3, p.91] or [Ko, p.42, Corollary 1.7.10] (where the $F$ there is our $H$ and the $\mathfrak{X}$ is our $Y$, see p.25) $Y$, and so $\left(\mathfrak{o}_{l} \otimes_{\mathbb{Z}_{l}} Y\right)^{\chi}$, are without finite submodules, from which together with [Wa, p.318] or [Ko, p.14, Proposition 1.4.1] it follows, firstly, that $\ell\left(\left(\left(\mathfrak{o}_{l} \otimes_{\mathbb{Z}_{l}} Y\right)^{\chi}\right)^{\Gamma}\right)=(1)$ and, secondly, that

$$
\ell\left(\left(\left(\mathfrak{o}_{l} \otimes_{\mathbb{Z}_{l}} Y\right)^{\chi}\right)_{\Gamma}\right)=l^{m_{\chi}^{\prime}} G_{\chi}^{*}(0) \mathfrak{o}_{l} .
$$

As $H_{\infty} / k$ is abelian, $e_{\chi}$ and $\gamma$ commute and so $\left(\left(\mathfrak{o}_{l} \otimes_{\mathbb{Z}_{l}} Y\right)^{\chi}\right)_{\Gamma}=\left(\mathfrak{o}_{l} \otimes_{\mathbb{Z}_{l}} Y_{\Gamma}\right)^{\chi}$. Thus we have arrived at

$$
\ell\left(\left(\mathfrak{o}_{l} \otimes_{\mathbb{Z}_{l}} Y_{\Gamma}\right)^{\chi}\right)=l^{m_{\chi}^{\prime}-m_{\chi}} L_{l}(1, \chi) \mathfrak{o}_{l} .
$$

We are left with showing $m_{\chi}=m_{\chi}^{\prime}$.

Let $\omega$ denote the Teichmüller character and $X_{\infty}$ the Galois group of the maximal abelian unramified $l$-extension of $H_{\infty}$. Then

$$
l^{m_{\chi}^{\prime}} G_{\chi}^{*}(T)=\text { characteristic polynomial of }\left(\mathfrak{o}_{l} \otimes_{\mathbb{Z}_{l}} X_{\infty}\right)^{\chi^{-1} \omega} \text { at } u(1+T)^{-1}-1 .
$$

This is $[\text { Ko, p.61, Theorem 1.9.9 }]^{6}$ with the following changes in the notation: in the diagram of fields on p. 56 in [Ko] we identify $E$ with our $k, F$ with our $H, \Delta$ with our $D, F_{\infty}$ with our $H_{\infty}$ and his $K$ with the fixed field of $\operatorname{ker}\left(\chi^{-1} \omega\right)$, which is totally complex since $\chi^{-1} \omega$ is odd as $\chi$ is even.

Combining the above formula with [Wi1, p.497, Theorem 1.4] gives the desired equality of the $\mu$-invariants:

$$
m_{\chi}^{\prime}=\mu\left(G_{\left(\chi^{-1} \omega\right)^{-1} \omega}\left(u(1+T)^{-1}-1\right)\right)=\mu\left(l^{m_{\chi}} G_{\chi}^{*}\left(u(1+T)^{-1}-1\right)\right)=m_{\chi} .
$$

\footnotetext{
${ }^{6} \mathrm{It}$ is also possible to get this by generalizing arguments from [Iw, §5], [Gr1, §2], [Li1, §§6,7], using [Wi1, Theorems 1.2,1.3].
} 
We finally consider the case $l=2$ and, to that end, look at the field diagram

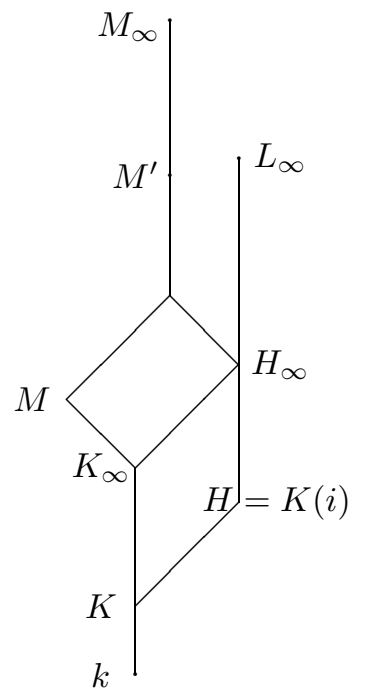

in which $i=\sqrt{-1}$.

$K_{\infty}, H_{\infty}$ are the cyclotomic 2-extensions of $K$ and $H$, respectively.

$M_{\infty}, M^{\prime}$ are the maximal abelian 2extensions of $K_{\infty}, K$, respectively, which are unramified outside 2 and the infinite primes.

$L_{\infty}$ is the maximal abelian unramified 2extension of $H_{\infty}$.

Set $X=G\left(L_{\infty} / H_{\infty}\right)$ and $Y=$ $G\left(M_{\infty} / K_{\infty}\right)$.

Because $K / \mathbb{Q}$ is abelian and not ramified at 2 , hence $K \cap \mathbb{Q}_{\infty}=\mathbb{Q}$, we are in the situation of $[\operatorname{Gr} 1, \S 2]$. Define $\psi=\chi^{-1} \omega$, where $\omega$ is, as before, the Teichmüller character. $^{7}$ Since $\chi$ is faithful on $G$, the odd character $\psi$ is faithful on $G(H / k)$ and is of type $S$. By [Wi1, Theorem 11.1, p.539]

$$
G_{\chi}^{*}\left(u(1+T)^{-1}-1\right)
$$

is the characteristic polynomial of $\gamma-1$ acting on $\left(F_{2} \otimes_{\mathbb{Z}_{2}} X\right)^{\psi}$.

The assumption $\psi \rho(\mathfrak{p}) \neq 1$ made in the quoted theorem is satisfied for each linear character $\rho$ of $G\left(k_{\infty} / k\right)$ and each prime $\mathfrak{p}$ of $k$ above 2. For $G\left(H k_{\infty} / K\right)$ is the inertia group for $\mathfrak{p}$ in $H k_{\infty} / k$ because $\mathfrak{p}$ is unramified in $K / k$ and totally ramified in $k_{\infty}(i) / k$. Since $\psi \rho$ is odd, its kernel does not contain $G\left(H k_{\infty} / K\right)$.

Define $X^{\prime}$ to be the Pontrjagin dual ${ }^{8}$ of the direct limit of the Sylow 2-subgroups $c l_{n}(2)$ of the class groups $c l_{n}$ in the $n^{\text {th }}$ field $H_{n}$ in the cyclotomic tower $H_{\infty} / H$, where the maps $c l_{n}(2) \rightarrow c l_{m}(2)$ are induced by the inclusions $H_{n} \longmapsto H_{m}$ for $m \geq n$. Then $X^{\prime}$ is quasi-isomorphic to $X$ as follows from [Iw, Theorem 11, p.266]; see also [Gr1, p.145]. Because of [Gr1, §2] $\left(F_{2} \otimes_{\mathbb{Z}_{2}} \dot{X}^{\prime}\right)^{\psi}$ and $\left(F_{2} \otimes_{\mathbb{Z}_{2}} Y\right)^{\chi}$ have the same characteristic polynomials. Here the $\operatorname{dot}$ on $X^{\prime}$ refers to a new action $\circ$ of $\gamma$ on $X^{\prime}$, namely $\gamma \circ x=u \cdot \gamma^{-1}(x), x \in X^{\prime}$.

Combining with (ii) we see that $G_{\chi}^{*}(T)$ is the characteristic polynomial of $\gamma-1$ on $\left(F_{2} \otimes_{\mathbb{Z}_{2}} Y\right)^{\chi}$.

By [Gr1, Proposition 8, p.151] the torsion subgroup of $\mathfrak{o}_{2} \otimes_{\mathbb{Z}_{2}} Y$ is pseudoisomorphic to $\Lambda /(2)[G(K / \mathbb{Q})]$, hence that of $\left(\mathfrak{o}_{2} \otimes_{\mathbb{Z}_{2}} Y\right)^{\chi}$ to $(\Lambda /(2)[G(K / \mathbb{Q})])^{\chi}$. As $\chi$ has multiplicity $[k: \mathbb{Q}]$ in the regular representation of $G(K / \mathbb{Q}), \quad\left(\mathfrak{o}_{2} \otimes_{\mathbb{Z}_{2}} Y\right)^{\chi}$ has $\mu$-invariant $2^{[k: \mathbb{Q}]}$ and thus characteristic polynomial $2^{[k: \mathbb{Q}]} G_{\chi}^{*}(T)$. From $[\mathrm{Gr} 3$, pp.93-94] we know that $Y$ has no non-trivial finite $\Lambda$-submodules. As in the odd case we deduce, by (i),

$$
\ell\left(\left(\mathfrak{o}_{2} \otimes_{\mathbb{Z}_{2}} Y_{\Gamma}\right)^{\chi}\right)=2^{[k: \mathbb{Q}]} G_{\chi}^{*}(0)=L_{2}(1, \chi) .
$$

${ }^{7}$ So the role of $\psi$ and $\chi$ is the opposite of [Wi1, Gr1].

${ }^{8} \operatorname{In}[\mathrm{Gr} 1] X$ and $X^{\prime}$ have just the opposite meaning. 
Now $Y_{\Gamma}=G\left(M^{\prime} / K\right)$ is an extension of $G(M / K)$ by $G\left(M^{\prime} / M\right)$. We show

$$
G\left(M^{\prime} / M\right) \simeq \mathbb{Z} /(2)[G(K / \mathbb{Q})]
$$

as $G(K / \mathbb{Q})$-modules and obtain, on taking $\chi$-eigenspaces, $\left(\mathfrak{o}_{2} \otimes_{\mathbb{Z}_{2}} G\left(M^{\prime} / M\right)\right)^{\chi} \simeq$ $\left(\mathfrak{o}_{2} /(2)\right)^{[k: \mathbb{Q}]}$, from which the theorem follows.

Proof of (iii). Denote by $\tilde{M}^{\prime}, \tilde{M}$ the maximal abelian extensions of $K$ which are unramified outside 2 and infinity, respectively outside 2 only. Let $\bar{E}^{\prime}, \bar{E}$ be the closures of the units $E$ of $K$ in $\Sigma \times \prod_{\mathfrak{P} \mid 2} U_{\mathfrak{P}}, \prod_{\mathfrak{P} \mid 2} U_{\mathfrak{P}}$, respectively. Here $U_{\mathfrak{P}}$ is the unit group in the completion $K_{\mathfrak{P}}$ of $K$ at $\mathfrak{P}$ and $\Sigma$ is the direct product of cyclic groups $C_{v}=\{ \pm 1\}$ with $v$ running through the real infinite primes of $K$. The map $E \rightarrow U_{\mathfrak{P}}$ is induced by the embedding $K \rightarrow K_{\mathfrak{P}} ; E \rightarrow C_{v}$ is the signature of $v$ on $E$. From [Wa, Corollary 13.6, p.268] we get the exact sequence $\bar{E} \longmapsto \prod_{\mathfrak{P} \mid 2} U_{\mathfrak{P}} \rightarrow$ $G\left(\tilde{M} / K_{h}\right)$ with $K_{h}$ denoting the Hilbert class field of $K$. In the same way, namely by replacing $U^{\prime}$ by $\Sigma \times \prod_{\mathfrak{P} \mid 2} U_{\mathfrak{P}}$ and $U^{\prime \prime}$ by $\prod_{v \text { real }} \mathbb{R}_{>0}^{\times} \times \prod_{v \text { complex }} \mathbb{C}^{\times} \times \prod_{\mathfrak{P} \nmid 2} U_{\mathfrak{P}}$ in [Wa, p.268], we obtain $\bar{E}^{\prime} \longmapsto \Sigma \times \prod_{\mathfrak{P} \mid 2} U_{\mathfrak{P}} \rightarrow G\left(\tilde{M}^{\prime} / K_{h}\right)$. Observe that both sequences are $G(K / \mathbb{Q})$-module sequences. They yield the commutative diagram

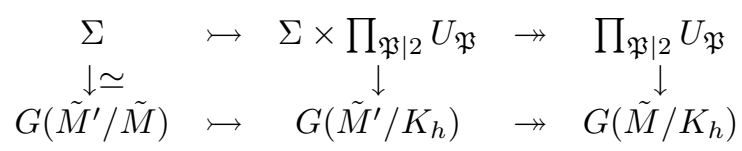

with surjective vertical maps. So our claim follows on taking 2-parts, because $\Sigma \simeq \mathbb{Z} /(2)[G(K / \mathbb{Q})]$

\section{REFERENCES}

[Br] Brumer, A., On the units of algebraic number fields. Mathematika 14 (1967), 121-124 MR 36:3746

[Ch1] Chinburg, T., On the Galois stucture of algebraic integers and S-units. Inventiones math. 74 (1983), 321-349 MR 86c:11096

[Ch2] Derivatives of L-functions at $s=0$. Compositio Math. 48 (1983), 119-127 MR 84k:12005

[Ch3] _ Exact sequences and Galois module structure. Annals of Math. 121 (1985), 351376 MR 86j:11115; MR 89i: 11119

[Fr1] Fröhlich, A., Galois Module Structure of Algebraic Integers. Springer-Verlag (1983) MR 85h:11067

[Fr2] L L-values at zero and multiplicative Galois module structure (also Galois Gauss sums and additive Galois module structure). J. Reine Angew. Math. 397 (1989), 42-99 MR 90g:11157

[Gc] Greither, C., Class groups of abelian fields and the main conjecture. Ann. Inst. Fourier 42 (1992), 449-499 MR 93j:11071

[GH] Greither, C. and Holland, D., Chinburg's third invariant in the defect class group for abelian extensions of $\mathbb{Q}$. Preprint December 1993

[Gr1] Greenberg, R., On p-adic L-functions and cyclotomic units. Nagoya Math. J. 67 (1977), 139-158 MR 56:2964

[Gr2] On p-adic Artin L-functions. Nagoya Math. J. 89 (1983), 77-87 MR 85b:11104

[Gr3] , On the structure of certain Galois groups. Inventiones math. 47 (1978), 85-99 MR 80b: 12007

[GW] Gruenberg, K. W. and Weiss, A., Galois invariants for units. Proc. LMS 70 (1995), 264284 MR 96f: 11148

[Iw] Iwasawa, K., On $\mathbb{Z}_{l}$-extensions of algebraic number fields. Annals of Math. 98 (1973), 246-326 MR 50:2120

[Ko] Kolster, M., K-theory and Arithmetic. Fields Institute Monographs, AMS; in preparation 
[Li1] Lichtenbaum, S., On the values of zeta and L-functions: I. Annals of Math. 96 (1972), 338-360 MR 50:12975

[Li2] - Values of zeta and L-functions at zero. Asterisque 24-25 (1975), 133-138 MR 53:5538

[Ma] Martinet, J., Character theory and Artin L-functions. In 'Algebraic Number Fields', Proceedings of the Durham Symposium 1975, ed. A. Fröhlich. Academic Press London (1977) MR 56:5502

[MW] Mazur, B. and Wiles, A., Class fields of abelian extensions of $\mathbb{Q}$. Inventiones math. 76 (1984), 179-330 MR 85m:11069

[RW1] Ritter, J. and Weiss, A., On the local Galois structure of S-units., in Algebra and Number Theory, Proceedings of a Conference held at the Institute for Experimental Mathematics, University of Essen, Germany, December 2-4, 1992 (eds. G. Frey, J. Ritter), de Gruyter Proceedings in Mathematics (1994), 229-245 MR 95e:11120

[RW2] _ A Tate sequence for global units. Compositio Math. 102 (1996), 147-178 CMP $96: 14$

[Se] Serre, J. P., Corps Locaux. Hermann, Paris (1962) MR 27:133

[So] Solomon, D., On Lichtenbaum's conjecture in the case of number fields. Ph.D. thesis, Brown University 1988

[St] Stark, H.-M., Values of L-functions at $s=1$. I. L-functions for quadratic forms. II. Artin L-functions with rational characters. III. Totally real fields and Hilbert's twelfth problem. IV. First derivatives at $s=0$. Advances in Math. 7 (1971), 301-343, 17 (1975), 60-92, 22 (1976), 64-84, 35 (1980), 197-235 MR 44:6620; MR 52:3082; MR 55:10427; MR 81f: 10054

[Ta1] Tate, J., The cohomology groups of tori in finite Galois extensions of number fields. Nagoya Math. J. 27 (1966), 709-719 MR 34:7495

[Ta2] Les Conjectures de Stark sur les Fonctions L d'Artin en $s=0$. Progress in Math. 47, Birkhäuser (1984) MR 86e: 11112

[Wa] Washington, L., Introduction to Cyclotomic Fields. Springer-Verlag (1982) MR 85g:11001

[We] Weiss, A., Multiplicative Galois module structure. Fields Institute Monographs 5, AMS (1996) CMP 96:11

[Wi1] Wiles, A., The Iwasawa conjecture for totally real fields. Annals of Math. 131 (1990), 493-540 MR 91i: 11163

[Wi2] _ On a conjecture of Brumer. Annals of Math. 131 (1990), 555-565 MR 91i:11164

Institut für Mathematik Der Universität, D-86135 Augsburg, Germany

Department of Mathematics, University of Alberta, Edmonton, Canada T6G 2G1 